\title{
Catalytic Cycloisomerization onto a Carbonyl Oxygen
}

\author{
Shicheng Shi,${ }^{\dagger}$ Jonathan L. Kuo,${ }^{\dagger}$ Tao Chen $^{\dagger}$ and Jack R. Norton* ${ }^{\dagger}$ \\ 'Department of Chemistry, Columbia University, 3000 Broadway, New York, New York 10027, \\ United States \\ jrn11@columbia.edu
}

\section{Supplementary Information}

Table of Contents 1

Experimental Procedures and Characterization Data 2

- Reaction Optimization 2

- Preparation of Starting Materials $\quad 8$

- Cycloisomerization under Cobaloxime/ $\mathrm{H}_{2}$ condition 17

- Cycloisomerization under salen-Co(III)/silane condition 22

- Mechanistic Studies 31

References

${ }^{1} \mathrm{H}$ and ${ }^{13} \mathrm{C}$ NMR Spectra

\section{Corresponding Author:}

Prof. Dr. J. Norton

Department of Chemistry

Columbia University, 3000 Broadway

New York, New York 10027, United States

E-mail: jrn11@columbia.edu 
All starting materials reported in the manuscript have been previously described in literature or prepared by the method reported previously. ${ }^{1-7}$ All experiments involving cyclization reaction were performed under argon in glovebox unless stated otherwise. All solvents were purchased at the highest commercial grade and used as received or after purification by passing through activated alumina columns or distillation from $\mathrm{Na} / \mathrm{benzophenone} \mathrm{under} \mathrm{nitrogen.} \mathrm{All} \mathrm{solvents}$ were deoxygenated prior to use. All other chemicals were purchased at the highest commercial grade and used as received. Reaction glassware was oven-dried at $140{ }^{\circ} \mathrm{C}$ for at least $24 \mathrm{~h}$ or flame-dried prior to use, allowed to cool under vacuum. All products were identified using ${ }^{1} \mathrm{H}$ NMR, ${ }^{13} \mathrm{C}$ NMR and/or HR-MS analysis. All yields refer to yields determined by ${ }^{1} \mathrm{H}$ NMR using an internal standard (nitromethane) unless stated otherwise. ${ }^{1} \mathrm{H}$ NMR and ${ }^{13} \mathrm{C}$ NMR spectra were recorded in $\mathrm{CDCl}_{3}, \mathrm{CD}_{2} \mathrm{Cl}_{2}$ and Acetone-d6 on Bruker 500 Ascend, DRX 500cor DRX 400 spectrometer. All coupling constants (J) are reported in hertz $(\mathrm{Hz})$. Abbreviations are: s, singlet; d, doublet; t, triplet; q, quartet; br s, broad singlet. High-resolution mass spectra (HRMS) were measured on a 7T Bruker Daltonics FT-MS instrument by electrospray ionization (ESI) probe. All flash chromatography was performed using silica gel, $60 \AA$, 300 mesh. TLC analysis was carried out on glass plates coated with silica gel $60 \mathrm{~F} 254,0.2 \mathrm{~mm}$ thickness. The plates were visualized using a $254 \mathrm{~nm}$ ultraviolet lamp or aqueous potassium permanganate solutions. ${ }^{1} \mathrm{H}$ NMR, ${ }^{13} \mathrm{C}$ NMR and HRMS data are given for all compounds in the Supporting Experimental for characterization purposes. ${ }^{1} \mathrm{H}$ NMR, ${ }^{13} \mathrm{C}$ NMR and HRMS data are reported for all new compounds.

Deuterated benzene $\left(\mathrm{C}_{6} \mathrm{D}_{6}\right)$ was distilled from molten potassium \& benzophenone ketyl. Benzene $\left(\mathrm{C}_{6} \mathrm{D}_{6}\right)$ and tetrahydrofuran (THF) were distilled from sodium-benzophenone ketyl. $\mathrm{CpCr}(\mathrm{CO})_{3} \mathrm{H}$, and $\mathrm{Co}\left(\mathrm{dmgBF}_{2}\right)_{2}(\mathrm{THF})_{2}$ were synthesized according to the literature procedures $^{1-2}$ and stored in an argon atmosphere glovebox $(\mathrm{O} 2<1 \mathrm{ppm})$.

\section{Detailed Optimization Experiments}

General Procedure: The cobalt or chromium catalysts and N-vinyl indole $1(0.2 \mathrm{mmol})$ were weighed in a glass vial and transferred to a Fisher-Porter tube equipped with a magnetic stir-bar using $4 \mathrm{~mL}$ of dry and degassed benzene. The tube was then placed behind an appropriate blast shield before it was pressurized to $2.0 \mathrm{~atm}$ of $\mathrm{H}_{2}$. The vessel was then immediately vented and repressurized and this operation was repeated twenty times, to push out the inert atmosphere. The 
vessel was pressurized to 2.0 atm of $\mathrm{H}_{2}$ for 30 seconds. If no change in pressure was observed, the tube was pressurized to 4.8 atm or 6.0 atm of $\mathrm{H}_{2}$, and stirred at $60{ }^{\circ} \mathrm{C}$ or $90{ }^{\circ} \mathrm{C}$ for 3 days. (NOTE: The pressurized Fisher-Porter tube should always be placed behind an appropriate blast shield!!!) After the indicated time, the Fisher-Potter tube was cooled to room temperature, $\mathrm{H}_{2}$ gas was vented slowly, then the Fisher-Porter tube was disassembled. The crude reaction mixture was filtered through a short silica gel plug, and the plug was washed with ethyl acetate. The resulting filtrate was concentrated and the crude reaction mixture was analysed by ${ }^{1} \mathrm{H} \mathrm{NMR}$ $\left(\mathrm{CDCl}_{3}, \mathrm{CD}_{2} \mathrm{Cl}_{2}, \mathrm{C}_{6} \mathrm{D}_{6}\right.$ or $\left.\mathrm{CD}_{3} \mathrm{C}(\mathrm{O}) \mathrm{CD}_{3}\right)$ to determine product distribution and selectivity with internal standard.

Table S1. Initial Optimization Studies Under Cobaloxime/ $\mathbf{H}_{2}{ }^{a}$
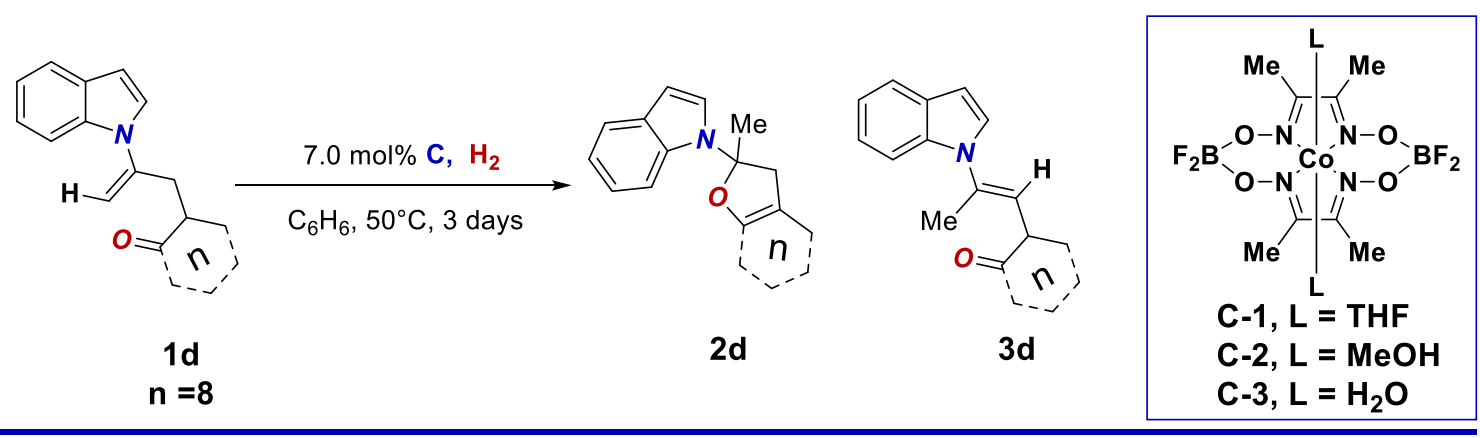

\begin{tabular}{|c|c|c|c|c|}
\hline entry & Substrate & Catalyst & Conversion $(\%)^{b}$ & Ratio $(\mathbf{2}: \mathbf{3})^{b}$ \\
\hline 1 & $1 d$ & C-1 & 86 & $55: 31$ \\
\hline 2 & $1 d$ & $\mathrm{C}-2$ & 45 & $18: 27$ \\
\hline 3 & $1 d$ & $C-3$ & 47 & $31: 16$ \\
\hline 4 & $1 b, n=5$ & C-1 & 82 & $14: 68$ \\
\hline 5 & 1a, $n=6$ & C-1 & 88 & $40: 48$ \\
\hline 6 & $1 c, n=7$ & C-1 & 84 & $48: 36$ \\
\hline 7 & $1 e, n=12$ & C-1 & 87 & $49: 38$ \\
\hline 8 & $1 d$ & $\mathrm{CpCr}(\mathrm{CO})_{3} \mathrm{H}$ & 29 & $<1: 27$ \\
\hline $9^{c}$ & $1 d$ & C-1 & 93 & $61: 32$ \\
\hline $10^{d}$ & $1 d$ & C-1 & 95 & $69: 26$ \\
\hline 11 & $1 d$ & No $[\mathrm{Co}]$ & $<5$ & - \\
\hline $12^{e}$ & $1 \mathrm{~d}$ & C-1 & $<5$ & - \\
\hline
\end{tabular}


${ }^{a}$ Conditions: 1 d $(0.2 \mathrm{mmol}), 7.0 \mathrm{~mol} \% \mathbf{C - 1}, 4.8$ atm $\mathrm{H}_{2}$, benzene $(0.05 \mathrm{M}), 50^{\circ} \mathrm{C}$, three days. ${ }^{b}$ Determined by ${ }^{1} \mathrm{H}$ NMR with internal standard. ${ }^{c} 20 \mathrm{~mol} \% \mathrm{C}-1$ was used. ${ }^{d}$ under $6.1 \mathrm{~atm} \mathrm{H}_{2} .{ }^{e}$ under Ar without $\mathrm{H}_{2}$.

\section{Optimization Studies With Co(III)-salen/silane}

General Procedure: In the glove box, to an oven-dried $6 \mathrm{~mL}$ vial equipped with a stir bar was Added the indole substrates $1(0.1 \mathrm{mmol})$ and $\mathrm{Co}(\mathrm{III})$-salen $(6 \mathrm{~mol} \%$ or $12 \mathrm{~mol} \%)$. This was followed by the addition of a stock solution of silane ( $6 \mathrm{~mol} \%$ or $12 \mathrm{~mol} \%)(1.0 \mathrm{~mL})$. The vial was sealed with a white cap, and further sealed using black tape. The reaction mixture was removed from glove box, put in pre-heated oil bath $\left(60{ }^{\circ} \mathrm{C}\right.$ or indicated temperature) for $16 \mathrm{~h}$. After the indicated time, the reaction mixture was cooled down to room temperature, filtered through a short plug of silica gel, and eluted with EtOAc. The resulting filtrate was concentrated and the crude reaction mixture was analysed by ${ }^{1} \mathrm{H} N M R\left(\mathrm{CDCl}_{3}, \mathrm{CD}_{2} \mathrm{Cl}_{2}\right.$ or $\left.\mathrm{CD}_{3} \mathrm{C}(\mathrm{O}) \mathrm{CD}_{3}\right)$ to determine product distribution and selectivity. Ratio is referred to cycloisomerization (2)/isomerization (3).

Figure S1. Structure of Co(III)-salen used for optimization

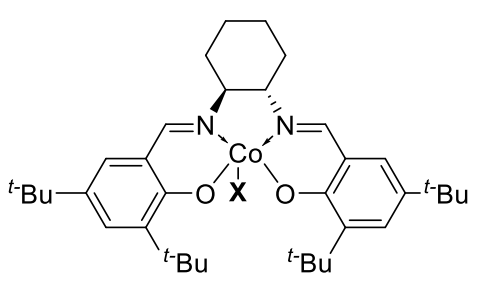

A: $\mathrm{X}=\mathrm{Cl}$

$\mathrm{B}: \mathrm{X}=\mathrm{Br}$

C: $X=1$

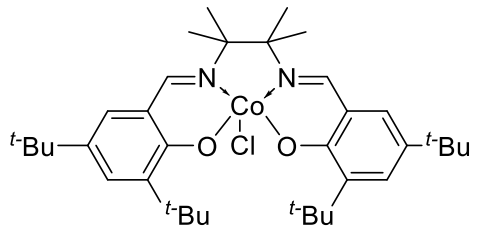

H: $51 \%$

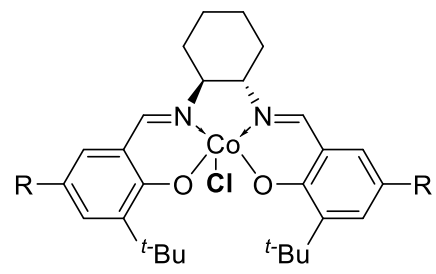

D: $\mathrm{R}=\mathrm{H}$

$\mathrm{E}: \mathrm{R}=\mathrm{OMe}$

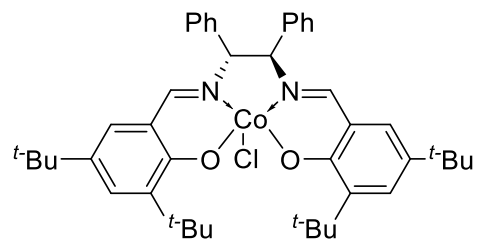

I: $94 \%$

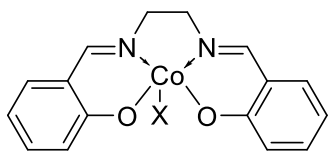

$F: X=C l$

$\mathbf{G}: \mathrm{X}=\mathrm{Br}$

The Co(III)-salen complexes A-I were prepared according to known procedures. ${ }^{3,4}$ Silane was used as a stock solution in Benzene. 
Table S2 Investigation of the temperature and concentration: ${ }^{a}$

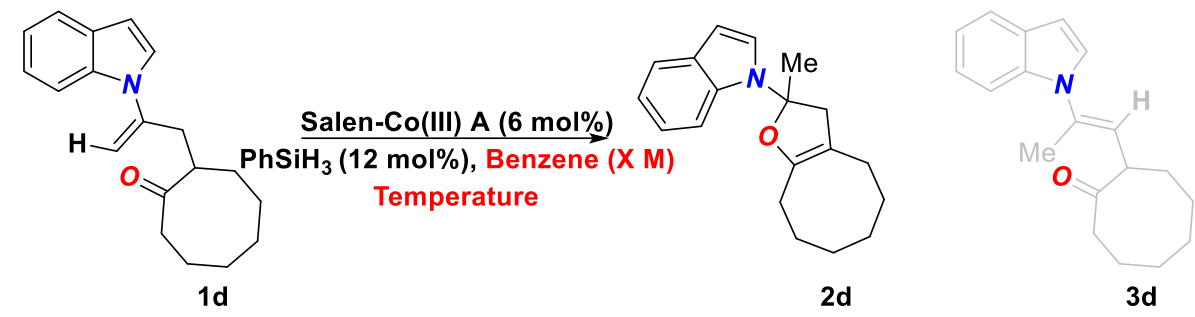

\begin{tabular}{ccccc}
\hline Entry & Temperature & Concentration & Conv. $^{b}(\%)$ & Ratio of $2 \mathrm{~d}: 3 \mathrm{~d}^{b}$ \\
\hline 1 & $23^{\circ} \mathrm{C}$ & $0.1 \mathrm{M}$ & 10 & $>20: 1$ \\
2 & $40^{\circ} \mathrm{C}$ & $0.1 \mathrm{M}$ & 45 & $6.7: 1$ \\
$\mathbf{3}$ & $\mathbf{6 0}^{\circ} \mathbf{C}$ & $\mathbf{0 . 1} \mathrm{M}$ & $\mathbf{8 0}$ & $\mathbf{4 . 5 : 1}$ \\
4 & $80^{\circ} \mathrm{C}$ & $0.1 \mathrm{M}$ & $>95$ & $2.1: 1$ \\
5 & $60^{\circ} \mathrm{C}$ & $0.01 \mathrm{M}$ & 78 & $3.5: 1$ \\
6 & $60^{\circ} \mathrm{C}$ & $0.5 \mathrm{M}$ & 75 & $3.3: 1$ \\
\hline
\end{tabular}

${ }^{a}$ Conditions: $1 \mathbf{d}(0.1 \mathrm{mmol}), 6.0 \mathrm{~mol} \% \mathrm{~A}, 12 \mathrm{~mol} \% \mathrm{PhSiH}_{3}$, benzene, temperature, $16 \mathrm{~h} .{ }^{b}$ Determined by ${ }^{1} \mathrm{H}$ NMR with internal standard.

Table S3. Investigation of silane as reductant: ${ }^{a}$

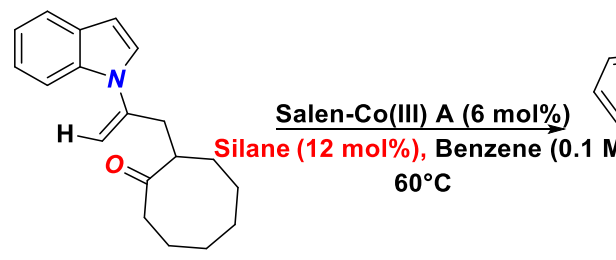

1d

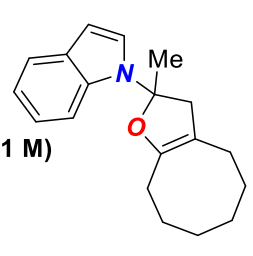

2d

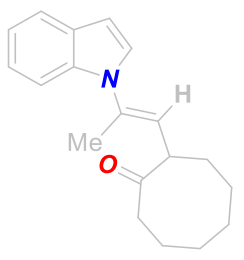

3d

\begin{tabular}{cccc}
\hline Entry & Silane & Conv. $^{b}(\%)$ & ${\text { Ratio of } 2 \mathrm{~d}: 3 \mathrm{~d}^{b}}^{b}$ \\
\hline 1 & $\mathrm{Et}_{3} \mathrm{SiH}$ & 10 & $>20: 1$ \\
2 & $(\mathrm{EtO})_{3} \mathrm{SiH}$ & 45 & $6.7: 1$ \\
3 & $\mathrm{Ph}_{3} \mathrm{SiH}$ & 80 & $4.5: 1$ \\
4 & $\mathrm{PHMS}$ & 33 & $3.1: 1$ \\
5 & $\mathrm{PhSiMeH}_{2}$ & 28 & $2.6: 1$ \\
$\mathbf{6}$ & $\mathbf{P h}_{2} \mathrm{SiH}_{2}$ & $\mathbf{7 8}$ & $\mathbf{6 . 5 : 1}$ \\
7 & $\mathrm{PhSiH}_{3}$ & 80 & $4.5: 1$ \\
\hline
\end{tabular}


${ }^{a}$ Conditions: $1 \mathrm{~d}(0.1 \mathrm{mmol}), 6.0 \mathrm{~mol} \% \mathrm{~A}, 12 \mathrm{~mol} \%$ silane, benzene $(0.1 \mathrm{M}), 60^{\circ} \mathrm{C}, 16 \mathrm{~h} .{ }^{b}$ Conversion and ratio of $\mathbf{2 d : 3 d}$ determined by ${ }^{1} \mathrm{H}$ NMR with internal standard.

Table S4. Investigation of salen-Co(III)-X catalyst: ${ }^{a}$
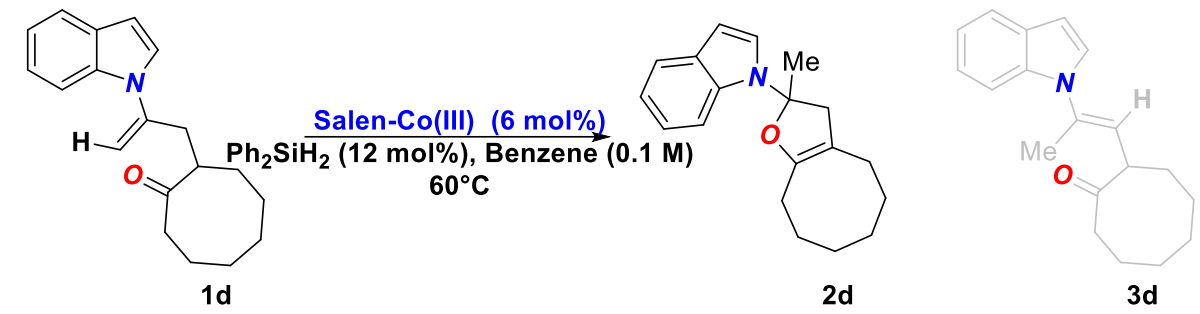

\begin{tabular}{cccc}
\hline Entry & Salen-Co(III) & Conv. $^{b}(\%)$ & ${\text { Ratio of } 2 \mathrm{~d}: 3 \mathrm{~d}^{b}}^{b}$ \\
\hline 1 & A & 91 & $6.5: 1$ \\
2 & B & 90 & $9.2: 1$ \\
$\mathbf{3}^{c}$ & B & $>\mathbf{9 5}$ & $\mathbf{8 . 5 : 1}$ \\
4 & C & 89 & $6.0: 1$ \\
5 & D & 35 & $3.4: 1$ \\
6 & E & 21 & $4.5: 1$ \\
7 & F & 37 & $2.4: 1$ \\
8 & G & 43 & $3.2: 1$ \\
9 & H & 51 & $12: 1$ \\
$10^{d}$ & H & 90 & $4.2: 1$ \\
11 & I & 94 & $2.3: 1$
\end{tabular}

${ }^{a}$ Conditions: 1d $(0.1 \mathrm{mmol}), 6.0 \mathrm{~mol} \%$ salen-Co(III), $12 \mathrm{~mol} \% \mathrm{Ph}_{2} \mathrm{SiH}_{2}$, benzene $(0.1 \mathrm{M}), 60^{\circ} \mathrm{C}, 16 \mathrm{~h}$. ${ }^{b}$ Conversion and ratio of $\mathbf{2 d : 3 d}$ determined by ${ }^{1} \mathrm{H}$ NMR with internal standard. ${ }^{c} 12.0 \mathrm{~mol} \% \mathbf{B}$ was used. ${ }^{c}$ under $80^{\circ} \mathrm{C}$

Table S5 Investigation of ring size of tethered cyclic ketones: ${ }^{a}$

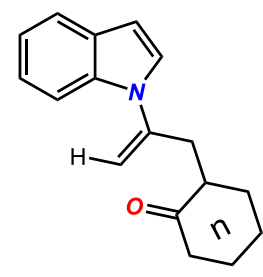

1

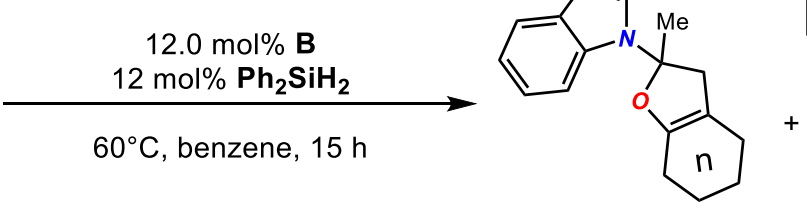

2

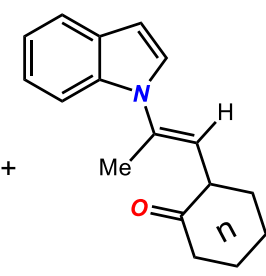

3 


\begin{tabular}{cccc}
\hline Entry & $\mathrm{n}$ & Conv. $^{b}(\%)$ & ${\text { Ratio of } 2: 3^{b}}^{b}$ \\
\hline 1 & 5 & 67 & $<1: 67$ \\
2 & 6 & 80 & $1.7: 1$ \\
$\mathbf{3}$ & $\mathbf{7}$ & $\mathbf{9 4}$ & $\mathbf{7 . 6 : 1}$ \\
$\mathbf{4}$ & $\mathbf{8}$ & $\mathbf{9 5}$ & $\mathbf{8 . 5 : 1}$ \\
5 & 9 & 94 & $3.3: 1$
\end{tabular}

${ }^{a}$ Conditions: 1 (0.2 mmol), $12.0 \mathrm{~mol} \% \mathbf{B}, 12 \mathrm{~mol} \% \mathrm{Ph}_{2} \mathrm{SiH}_{2}$, benzene $(0.1 \mathrm{M}), 60^{\circ} \mathrm{C}, 16 \mathrm{~h} .{ }^{b}$ Conversion and ratio of 2:3 determined by ${ }^{1} \mathrm{H}$ NMR with internal standard. 


\section{Preparation of Starting Materials}<smiles>C=C(Br)CC1CCCC1=O</smiles>

s1<smiles>C=C(Br)CC1CCCCC1=O</smiles>

S2<smiles>C=C(Br)CC1CCCCCC1=O</smiles>

S3<smiles>C=C(Br)CC1CCCCCCC1=O</smiles>

S4

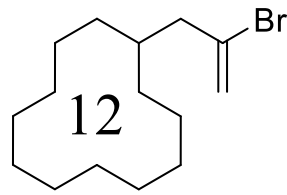

S5

S1-S2, S3-S5 were prepared according to reported procedures. ${ }^{5,6}$

\section{General procedure for preparation of S1 and S2: ${ }^{5}$}

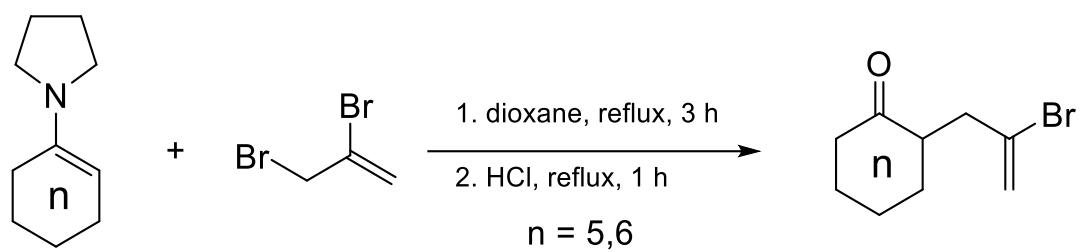

Enamine $(5.0 \mathrm{mmol})$ was added to $5 \mathrm{~mL}$ dioxane in a $20 \mathrm{~mL}$ oven dried $\mathrm{RBF}$, then 2,3 dibromoprop-1-ene $(0.55 \mathrm{~mL}, 0.55 \mathrm{mmol})$ was added dropwise, the reaction mixture was stirred at reflux for 3 hours. The reaction mixture was then cooled to room temperature, followed by addition of $1 \mathrm{~N} \mathrm{HCl}(1 \mathrm{~mL})$. The reaction was heated at reflux for additional 1 hour, TLC indicated the disappearance of the starting material. Solvent was removed in vacuo, and the product was purified via chromatography (hexane/EtOAc $=50: 1-10: 1)$ afford the final product as colourless oil. (stained with aqueous potassium permanganate solutions)

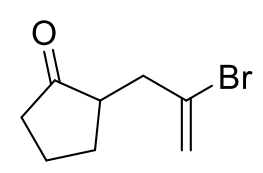

S1

2-(2-Bromoallyl)cyclopentan-1-one (S1): $5.0 \mathrm{mmol}$ scale, the product was purified through chromatography (hexane / EtOAc $=50: 1-10: 1$ ) afford as colourless oil. Yield $80 \%$ (804 mg). ${ }^{1} \mathrm{H}$ NMR (400 MHz, $\left.\mathrm{CDCl}_{3}\right) \delta 5.62(\mathrm{td}, J=$ 1.6, $0.7 \mathrm{~Hz}, 1 \mathrm{H}), 5.44(\mathrm{dd}, J=1.7,0.7 \mathrm{~Hz}, 1 \mathrm{H}), 2.95(\mathrm{dd}, J=15.0,4.0 \mathrm{~Hz}, 1 \mathrm{H})$, $2.54-2.21(\mathrm{~m}, 4 \mathrm{H}), 2.15(\mathrm{ddd}, J=18.8,10.4,8.8 \mathrm{~Hz}, 1 \mathrm{H}), 2.03$ (dddt, $J=13.6$, 9.0, 6.8, $2.4 \mathrm{~Hz}, 1 \mathrm{H}), 1.91-1.76(\mathrm{~m}, 1 \mathrm{H}), 1.51(\mathrm{dtd}, J=12.8,11.0,6.8 \mathrm{~Hz}, 1 \mathrm{H})$. Spectrum data match with previously reported.<smiles>C=C(Br)CC1CCCCC1=O</smiles>

S2
2-(2-Bromoallyl)cyclohexan-1-one (S2): $5.0 \mathrm{mmol}$ scale, the product was purified through chromatography (hexane $/$ EtOAc $=50: 1-10: 1$ ) afford as colourless oil. Yield $78 \%(842 \mathrm{mg}) .{ }^{1} \mathrm{H}$ NMR $\left(400 \mathrm{MHz}, \mathrm{CDCl}_{3}\right) \delta 5.60(\mathrm{td}, J=$ 
1.6, $0.8 \mathrm{~Hz}, 1 \mathrm{H}), 5.44(\mathrm{dd}, J=1.6,0.8 \mathrm{~Hz}, 1 \mathrm{H}), 3.00(\mathrm{dd}, J=14.8,4.2 \mathrm{~Hz}, 1 \mathrm{H}), 2.76-2.63(\mathrm{~m}$, $1 \mathrm{H}), 2.48-2.31(\mathrm{~m}, 2 \mathrm{H}), 2.27(\mathrm{dd}, J=14.8,9.0 \mathrm{~Hz}, 1 \mathrm{H}), 2.18(\mathrm{ddt}, J=11.7,5.9,3.0 \mathrm{~Hz}, 1 \mathrm{H})$, 2.10 (ddt, $J=12.3,6.3,3.0 \mathrm{~Hz}, 1 \mathrm{H}), 1.94-1.85(\mathrm{~m}, 1 \mathrm{H}), 1.78-1.58(\mathrm{~m}, 2 \mathrm{H}), 1.31-1.17(\mathrm{~m}$, 1H). Spectrum data match with previously reported.

\section{General procedure for preparation of S3-S5: 6}
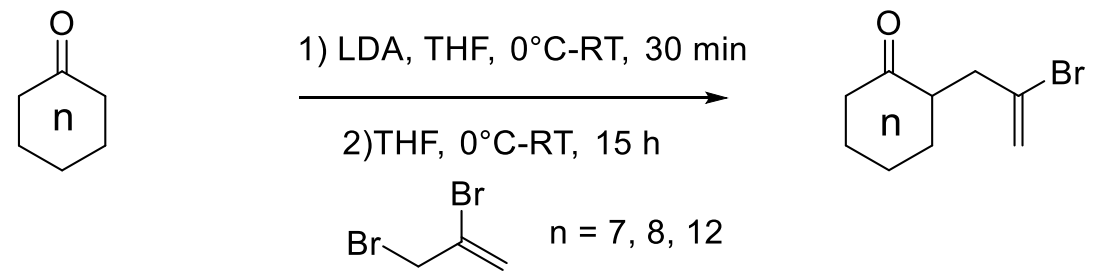

To a solution of fresh prepared LDA (5.5 mmol, 1.1 equiv), a solution of corresponding cyclic ketone $\left(5.0 \mathrm{mmol}, 1.0\right.$ equiv) in THF $(3.0 \mathrm{~mL})$ was added dropwise at $0^{\circ} \mathrm{C}$. The reaction was warmed to room temperature and stirred for $30 \mathrm{~min}$. Then 2,3-dibromoprop-1-ene $(0.55 \mathrm{~mL}, 0.55$ mmol) was added the reaction was stirred at room temperature for 15 hours. The reaction was quenched by $\mathrm{NH}_{4} \mathrm{Cl}(10 \mathrm{~mL})$ and extracted with $\mathrm{Et}_{2} \mathrm{O}(3 \times 15 \mathrm{~mL})$; the combined organic layer was further washed with brine $(20 \mathrm{~mL})$ and dried over $\mathrm{Na}_{2} \mathrm{SO}_{4}$. The solvent was removed, and the product was purified through chromatography (hexane / EtOAc $=50: 1-10: 1$ ) afford the final product as colourless oil. (stained with aqueous potassium permanganate solutions)

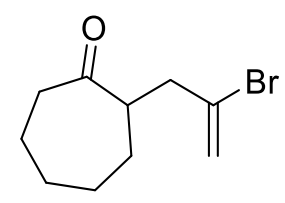

S3

2-(2-Bromoallyl)cycloheptan-1-one (S3) : $5.0 \mathrm{mmol}$ scale, the product was purified through chromatography (hexane / EtOAc $=50: 1-10: 1$ ) afford the as colourless oil. Yield $82 \%$ (947 mg). ${ }^{1} \mathrm{H}$ NMR (400 MHz, $\left.\mathrm{CDCl}_{3}\right) \delta 5.60(\mathrm{dd}, J$ $=1.8,0.9 \mathrm{~Hz}, 1 \mathrm{H}), 5.45(\mathrm{dd}, J=1.6,0.7 \mathrm{~Hz}, 1 \mathrm{H}), 2.96(\mathrm{dddd}, J=10.1,8.4$, 5.6, $3.0 \mathrm{~Hz}, 1 \mathrm{H}), 2.91-2.81(\mathrm{~m}, 1 \mathrm{H}), 2.58$ (dddd, $J=15.6,5.3,4.3,1.2 \mathrm{~Hz}$, 1H), 2.45 (ddd, $J=15.5,11.0,4.3 \mathrm{~Hz}, 1 \mathrm{H}), 2.37$ (ddd, $J=14.7,8.3,0.8 \mathrm{~Hz}, 1 \mathrm{H}), 1.92-1.79$ (m, $4 \mathrm{H}), 1.78-1.66(\mathrm{~m}, 1 \mathrm{H}), 1.56-1.45(\mathrm{~m}, 1 \mathrm{H}), 1.39-1.23(\mathrm{~m}, 2 \mathrm{H}) .{ }^{13} \mathrm{C} \mathrm{NMR}\left(101 \mathrm{MHz}, \mathrm{CDCl}_{3}\right)$ $\delta 214.2,132.6,49.6,43.5,42.9,29.9,29.3,29.0,23.9$. HRMS (ESI) m/z: $[\mathrm{M}+\mathrm{Na}]^{+}$Calcd for $\mathrm{C}_{10} \mathrm{H}_{15} \mathrm{BrONa} 230.0305$, found 396.1791.

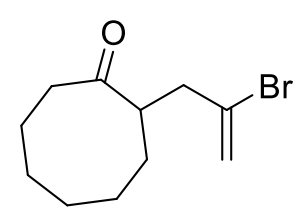

S4

2-(2-Bromoallyl)cyclooctan-1-one (S4) : $30.0 \mathrm{mmol}$ scale, the product was purified through chromatography (hexane / EtOAc $=50: 1-10: 1$ ) afford as colourless oil. Yield $75 \%(5.59 \mathrm{~g}) .{ }^{1} \mathrm{H} \mathrm{NMR}\left(400 \mathrm{MHz}, \mathrm{CDCl}_{3}\right) \delta 5.59-5.55$ 
(m, 1H), $5.41(\mathrm{~d}, J=1.7 \mathrm{~Hz}, 1 \mathrm{H}), 3.14(\mathrm{dtd}, J=10.4,7.1,3.6 \mathrm{~Hz}, 1 \mathrm{H}), 2.82$ (ddd, $J=14.4,7.4$, $1.1 \mathrm{~Hz}, 1 \mathrm{H}), 2.49$ (ddd, $J=14.2,7.8,3.2 \mathrm{~Hz}, 1 \mathrm{H}), 2.40$ (ddd, $J=14.4,6.7,1.0 \mathrm{~Hz}, 1 \mathrm{H}), 2.32$ (ddd, $J=14.1,10.9,3.2 \mathrm{~Hz}, 1 \mathrm{H}), 2.10$ (dddd, $J=17.6,10.9,5.4,3.3 \mathrm{~Hz}, 1 \mathrm{H}), 1.90$ (ddt, $J=13.7$, 7.1, $3.4 \mathrm{~Hz}, 1 \mathrm{H}), 1.85$ - $1.75(\mathrm{~m}, 1 \mathrm{H}), 1.72$ - 1.55 (m, 5H), 1.49 - 1.37 (m, 1H), 1.19 - 1.06 (m, $1 \mathrm{H}) .{ }^{13} \mathrm{C}$ NMR $\left(101 \mathrm{MHz}, \mathrm{CDCl}_{3}\right) \delta 218.2,132.1,119.0,47.6,43.5,43.3,32.5,28.0,25.0,24.9$, 24.6. HRMS (ESI) m/z: [M + H $]^{+}$Calcd for $\mathrm{C}_{11} \mathrm{H}_{18} \mathrm{BrO} 245.0541$, found 245.0547.

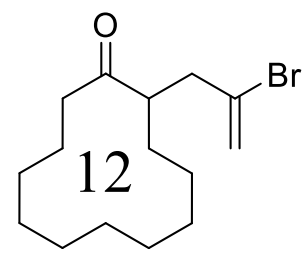

S5

2-(2-bromoallyl)cyclododecan-1-one : $5.0 \mathrm{mmol}$ scale, the product was purified through chromatography (hexane / EtOAc $=50: 1-10: 1)$ afford as colourless oil. Yield $66 \%$ (990 mg). ${ }^{1} \mathrm{H}$ NMR (400 $\left.\mathrm{MHz}, \mathrm{CDCl}_{3}\right) \delta 5.61-$ $5.59(\mathrm{~m}, 1 \mathrm{H}), 5.43(\mathrm{~d}, J=1.7 \mathrm{~Hz}, 1 \mathrm{H}), 3.07$ (p, $J=6.5 \mathrm{~Hz}, 1 \mathrm{H}), 2.80$ (ddd, $J$ $=17.0,9.9,3.2 \mathrm{~Hz}, 1 \mathrm{H}), 2.72(\mathrm{dd}, J=14.6,7.2 \mathrm{~Hz}, 1 \mathrm{H}), 2.45(\mathrm{dd}, J=14.7$, $7.3 \mathrm{~Hz}, 1 \mathrm{H}), 2.28$ (ddd, $J=17.0,7.6,3.5 \mathrm{~Hz}, 1 \mathrm{H}), 1.88$ (tq, $J=10.3,4.5,3.3 \mathrm{~Hz}, 1 \mathrm{H}), 1.67$ (q, $J$ $=7.6 \mathrm{~Hz}, 2 \mathrm{H}), 1.34-1.25(\mathrm{~m}, 15 \mathrm{H}) .{ }^{13} \mathrm{C} \mathrm{NMR}\left(101 \mathrm{MHz}, \mathrm{CDCl}_{3}\right) \delta 213.1,132.2,118.9,49.1$, 42.2 38.8, 28.5, 26.2, 25.9, 24.2, 23.9, 23.7, 22.4, 22.0, 21.9. HRMS (ESI) m/z: $[\mathrm{M}+\mathrm{H}]^{+}$Calcd for $\mathrm{C}_{15} \mathrm{H}_{26} \mathrm{BrO}$ 301.1167, found 301.1172.

\section{General procedure for preparation of $1 \mathrm{a}-1 \mathrm{p}:^{5}$}
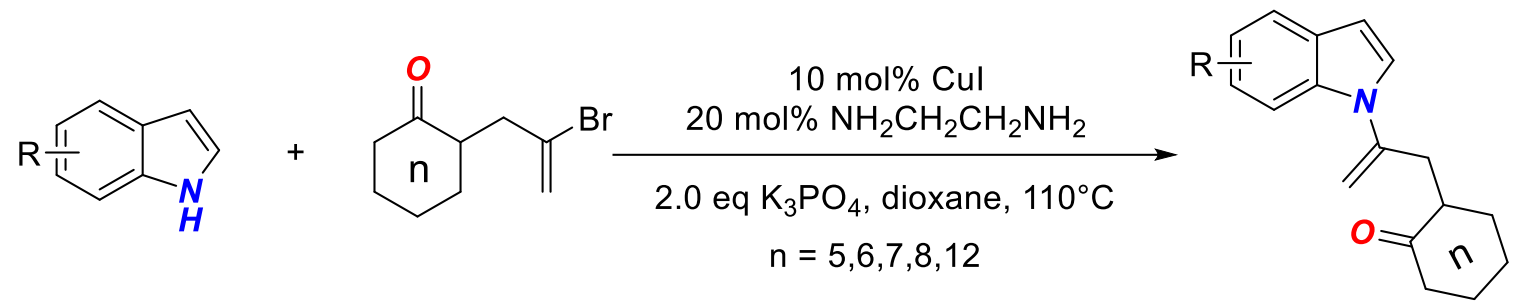

To a $20 \mathrm{~mL}$ pressure tube was added the subsituted-indole $(5.5 \mathrm{mmol})$ and then $\mathrm{K}_{3} \mathrm{PO}_{4}(2.12 \mathrm{~g}$, $5.0 \mathrm{mmol}), \mathrm{CuI}(95.0 \mathrm{mg}, 0.5 \mathrm{mmol}), 5 \mathrm{~mL}$ of degassed dioxane, and ethane-1,2- diamine (0.06 $\mathrm{mL}, 1.0 \mathrm{mmol})$. This was followed by the addition of vinyl-bromide $(1.0 \mathrm{mmol})$ in one portion. The pressure tube was sealed with a PTFE plug, and the mixture was heated at $110{ }^{\circ} \mathrm{C}$ for $24 \mathrm{~h}$. The mixture was cooled to room temperature, and ethyl acetate $(20 \mathrm{~mL})$ was added followed by filtration through a short plug of Celite. The organic phase was concentrated under reduced pressure, and the resulting residue was purified by column (toluene/DCM 50:1-10:1) to yield the titled compound $\mathbf{1}$ as a colorless oil. 


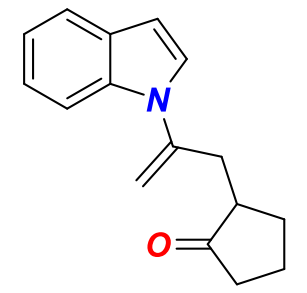

$1 \mathrm{~b}$

2-(2-(1H-indol-1-yl)allyl)cyclopentan-1-one(1b): $5.0 \mathrm{mmol}$ scale, the product was purified through chromatography (toluene/DCM 50:1-10:1) afford as colourless oil. Yield 57\% (681 mg). ${ }^{1} \mathrm{H}$ NMR (400 MHz, $\left.\mathrm{CD}_{3} \mathrm{C}(\mathrm{O}) \mathrm{CD}_{3}\right) \delta 7.64-7.53(\mathrm{~m}, 2 \mathrm{H}), 7.39(\mathrm{~d}, J=3.3 \mathrm{~Hz}, 1 \mathrm{H}), 7.19$ (ddd, $J=$ 8.5, 7.1, 1.2 Hz, 1H), 7.07 (ddd, $J=7.8,7.1,1.1 \mathrm{~Hz}, 1 \mathrm{H}), 6.57$ (dd, $J=3.4,0.7$ $\mathrm{Hz}, 1 \mathrm{H}), 5.34-5.27(\mathrm{~m}, 1 \mathrm{H}), 5.20(\mathrm{~s}, 1 \mathrm{H}), 3.21$ (ddd, $J=15.1,3.8,1.3 \mathrm{~Hz}$, 1H), 2.46 (dd, $J=15.1,9.8 \mathrm{~Hz}, 1 \mathrm{H}), 2.17$ (dddt, $J=18.3,8.2,2.6,1.6 \mathrm{~Hz}, 1 \mathrm{H}), 2.04-1.86$ (m, $4 \mathrm{H}), 1.71-1.52(\mathrm{~m}, 2 \mathrm{H}) .{ }^{13} \mathrm{C}$ NMR $\left(101 \mathrm{MHz}, \mathrm{CD}_{3} \mathrm{C}(\mathrm{O}) \mathrm{CD}_{3}\right) \delta$ 218.6, 144.4, 136.7, 130.2, $127.5,122.9,121.6,120.9,112.0,108.5,103.8,47.4,37.9,36.4,20.9$. Spectrum data match with what was previously reported. ${ }^{7}$

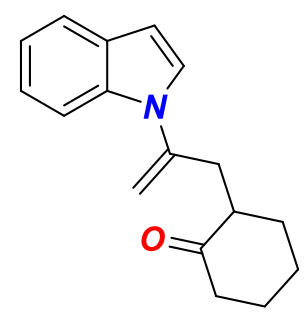

$1 \mathrm{a}$

2-(2-(1H-indol-1-yl)allyl)cyclohexan-1-one (1a): $5.0 \mathrm{mmol}$ scale, the product was purified through chromatography (toluene/DCM 50:1-10:1) afford as colourless oil. Yield 53\% (670 mg). ${ }^{1} \mathrm{H}$ NMR (400 MHz, $\left.\mathrm{CD}_{3} \mathrm{C}(\mathrm{O}) \mathrm{CD}_{3}\right) \delta 7.61-7.54(\mathrm{~m}, 2 \mathrm{H}), 7.36(\mathrm{~d}, J=3.3 \mathrm{~Hz}, 1 \mathrm{H}), 7.17(\mathrm{ddd}, J=$ 8.4, 7.0, 1.2 Hz, 1H), 7.07 (ddd, $J=7.9,7.0,1.0 \mathrm{~Hz}, 1 \mathrm{H}), 6.56(\mathrm{dd}, J=3.3$, $0.8 \mathrm{~Hz}, 1 \mathrm{H}), 5.24(\mathrm{~d}, J=0.7 \mathrm{~Hz}, 1 \mathrm{H}), 5.20(\mathrm{~s}, 1 \mathrm{H}), 3.29(\mathrm{ddd}, J=15.2,4.7$,

$1.3 \mathrm{~Hz}, 1 \mathrm{H}), 2.40(\mathrm{dd}, J=15.3,8.8 \mathrm{~Hz}, 1 \mathrm{H}), 2.35-2.23(\mathrm{~m}, 3 \mathrm{H}), 2.14$ (ddq, $J=12.4,5.7,3.3 \mathrm{~Hz}$, 1H), $2.02-1.94(\mathrm{~m}, 1 \mathrm{H}), 1.77(\mathrm{ddd}, J=11.5,4.4,2.3 \mathrm{~Hz}, 1 \mathrm{H}), 1.65-1.45(\mathrm{~m}, 2 \mathrm{H}), 1.36$ (qd, $J$ $=12.4,3.6 \mathrm{~Hz}, 1 \mathrm{H})$. HRMS (ESI) m/z: $[\mathrm{M}+\mathrm{H}]^{+}$Calcd for $\mathrm{C}_{18} \mathrm{H}_{22} \mathrm{NO} 268.1701$, found 268.1717 .

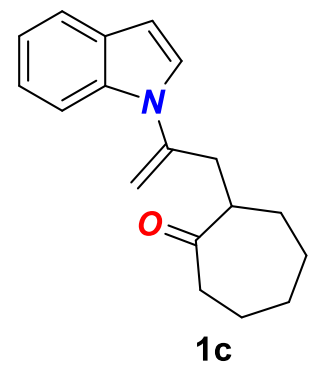

2-(2-(1H-indol-1-yl)allyl)cycloheptan-1-one(1c): $5.0 \mathrm{mmol}$ scale, the product was purified through chromatography (toluene/DCM 50:1-10:1) afford as colourless oil. Yield 76\% (1.02 g). ${ }^{1} \mathrm{H}$ NMR (400 MHz, $\left.\mathrm{CD}_{3} \mathrm{C}(\mathrm{O}) \mathrm{CD}_{3}\right) \delta 7.62-7.53(\mathrm{~m}, 2 \mathrm{H}), 7.37(\mathrm{~d}, J=3.3 \mathrm{~Hz}, 1 \mathrm{H}), 7.19$ (ddd, $J=$ 8.3, 7.0, $1.3 \mathrm{~Hz}, 1 \mathrm{H}), 7.09$ (ddd, $J=8.0,7.0,1.0 \mathrm{~Hz}, 1 \mathrm{H}), 6.59$ (dd, $J=3.4$, $0.8 \mathrm{~Hz}, 1 \mathrm{H}), 5.26(\mathrm{~s}, 1 \mathrm{H}), 5.22(\mathrm{~s}, 1 \mathrm{H}), 3.15(\mathrm{ddd}, J=15.2,5.8,1.1 \mathrm{~Hz}, 1 \mathrm{H})$, $2.58(\mathrm{dd}, J=15.4,8.0 \mathrm{~Hz}, 1 \mathrm{H}), 2.44(\mathrm{dddd}, J=9.7,8.3,5.9,3.4 \mathrm{~Hz}, 1 \mathrm{H}), 2.34$ (dd, $J=7.8,4.9$ $\mathrm{Hz}, 2 \mathrm{H}), 1.91-1.83(\mathrm{~m}, 1 \mathrm{H}), 1.80-1.66(\mathrm{~m}, 3 \mathrm{H}), 1.55-1.22(\mathrm{~m}, 4 \mathrm{H}) .{ }^{13} \mathrm{C} \mathrm{NMR}(101 \mathrm{MHz}$, $\left.\mathrm{CD}_{3} \mathrm{C}(\mathrm{O}) \mathrm{CD}_{3}\right) \delta 213.4,144.3,136.8,130.2,127.5,122.9,121.6,120.8,112.0,109.3,103.7,49.4$, 
43.4, 37.9, 30.8, 30.0, 29.2, 24.6. HRMS (ESI) m/z: $[\mathrm{M}+\mathrm{H}]^{+}$Calcd for $\mathrm{C}_{17} \mathrm{H}_{20} \mathrm{NO} 254.1545$, found 254.1551.

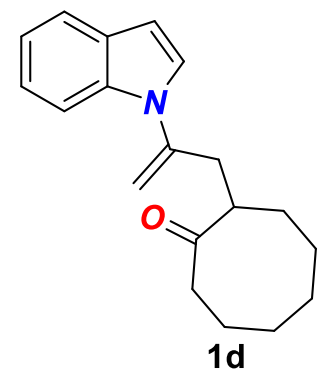

2-(2-(1H-indol-1-yl)allyl)cyclooctan-1-one (1d): $20.0 \mathrm{mmol}$ scale, the product was purified through chromatography (toluene/DCM 50:1-10:1) afford as colourless oil. Yield 80\% (4.50 g). ${ }^{1} \mathrm{H}$ NMR (400 MHz, $\left.\mathrm{CD}_{3} \mathrm{C}(\mathrm{O}) \mathrm{CD}_{3}\right) \delta 7.57$ (ddt, $\left.J=17.7,8.4,1.0 \mathrm{~Hz}, 2 \mathrm{H}\right), 7.37(\mathrm{~d}, J=3.3 \mathrm{~Hz}$, $1 \mathrm{H}), 7.18(\mathrm{ddd}, J=8.3,7.0,1.3 \mathrm{~Hz}, 1 \mathrm{H}), 7.07$ (ddd, $J=8.0,7.1,1.0 \mathrm{~Hz}, 1 \mathrm{H}$ ), $6.58(\mathrm{dd}, J=3.3,0.9 \mathrm{~Hz}, 1 \mathrm{H}), 5.21(\mathrm{t}, J=1.1 \mathrm{~Hz}, 1 \mathrm{H}), 5.17$ (s, 1H), 3.06 (ddd, $J=14.8,7.4,1.1 \mathrm{~Hz}, 1 \mathrm{H}), 2.70(\mathrm{ddd}, J=14.8,6.7,1.0 \mathrm{~Hz}, 1 \mathrm{H}), 2.61(\mathrm{dtd}, J=10.3,7.0,3.4$ $\mathrm{Hz}, 1 \mathrm{H}), 2.20-2.14(\mathrm{~m}, 2 \mathrm{H}), 1.971 .87(\mathrm{~m}, 1 \mathrm{H}), 1.82-1.72(\mathrm{~m}, 1 \mathrm{H}), 1.66-1.54(\mathrm{~m}, 3 \mathrm{H}), 1.50$ $-1.40(\mathrm{~m}, 3 \mathrm{H}), 1.35-1.26(\mathrm{~m}, 1 \mathrm{H}), 1.16-1.00(\mathrm{~m}, 1 \mathrm{H}) .{ }^{13} \mathrm{C}$ NMR $\left(101 \mathrm{MHz}, \mathrm{CD}_{3} \mathrm{C}(\mathrm{O}) \mathrm{CD}_{3}\right) \delta$ 216.1, 143.1, 135.8, 129.3, 126.6, 121.9, 120.7, 119.9, 111.1, 107.7, 102.8, 46.8, 41.9, 37.0, 31.9, 27.3, 24.8, 24.6, 24.4. HRMS (ESI) $\mathrm{m} / \mathrm{z}:[\mathrm{M}+\mathrm{H}]^{+}$Calcd for $\mathrm{C}_{19} \mathrm{H}_{24} \mathrm{NO} 282.1858$, found 282.1879 .

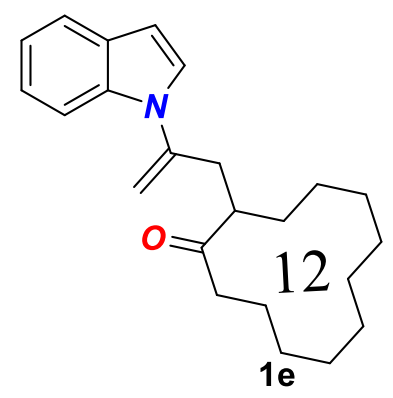

2-(2-(1H-indol-1-yl)allyl)cyclododecan-1-one (1e): $5.0 \mathrm{mmol}$ scale, the product was purified through chromatography (toluene/DCM 50:110:1) afford as colourless oil. Yield 62\% (1.05 g). ${ }^{1} \mathrm{H}$ NMR (400 MHz, $\left.\mathrm{CD}_{2} \mathrm{Cl}_{2}\right) \delta 7.65(\mathrm{~d}, J=7.9 \mathrm{~Hz}, 1 \mathrm{H}), 7.57(\mathrm{dd}, J=8.3,1.0 \mathrm{~Hz}, 1 \mathrm{H}), 7.28-$ $7.20(\mathrm{~m}, 2 \mathrm{H}), 7.15(\mathrm{ddd}, J=8.0,7.0,1.0 \mathrm{~Hz}, 1 \mathrm{H}), 6.63(\mathrm{dd}, J=3.3,0.8$ $\mathrm{Hz}, 1 \mathrm{H}), 5.26(\mathrm{~s}, 1 \mathrm{H}), 5.24(\mathrm{~d}, J=1.2 \mathrm{~Hz}, 1 \mathrm{H}), 3.03(\mathrm{ddd}, J=15.2,7.0$, $1.1 \mathrm{~Hz}, 1 \mathrm{H}), 2.72-2.61(\mathrm{~m}, 2 \mathrm{H}), 2.57$ (qd, $J=7.1,4.2 \mathrm{~Hz}, 1 \mathrm{H}), 2.16$ (ddd, $J=17.3,7.4,3.5 \mathrm{~Hz}$, $1 \mathrm{H}), 1.89-1.77(\mathrm{~m}, 1 \mathrm{H}), 1.68(\mathrm{ddd}, J=8.0,4.0,2.1 \mathrm{~Hz}, 2 \mathrm{H}), 1.39-1.19(\mathrm{~m}, 15 \mathrm{H}) .{ }^{13} \mathrm{C}$ NMR $\left(101 \mathrm{MHz}, \mathrm{CD}_{2} \mathrm{Cl}_{2}\right) \delta 212.4,143.0,135.9,129.2,126.4,122.1,120.8,120.1,111.2,108.7,103.0$, 48.5, 37.5, 35.8, 28.6, 26.0, 25.8, 23.9, 23.4, 23.0, 22.2, 21.7, 21.5. HRMS (ESI) m/z: [M + H] ${ }^{+}$ Calcd for $\mathrm{C}_{23} \mathrm{H}_{32} \mathrm{NO} 338.2484$, found 338.2488 .

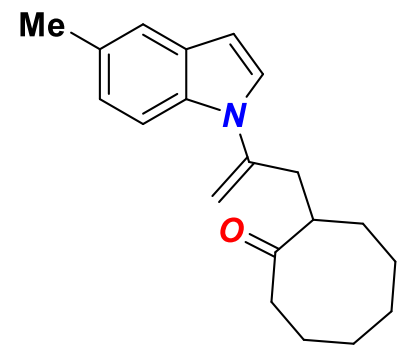

2-(2-(5-Methyl-1H-indol-1-yl)allyl)cyclooctan-1-one (1f): $3.0 \mathrm{mmol}$ scale, the product was purified through chromatography (toluene/DCM 50:1-10:1) afford as colourless oil. Yield 82\% (726 mg). ${ }^{1} \mathrm{H}$ NMR (400

\section{SI-12}


$\left.\mathrm{MHz}, \mathrm{CD}_{3} \mathrm{C}(\mathrm{O}) \mathrm{CD}_{3}\right) \delta 7.43(\mathrm{~d}, J=8.5 \mathrm{~Hz}, 1 \mathrm{H}), 7.37(\mathrm{dt}, J=1.7,0.9 \mathrm{~Hz}, 1 \mathrm{H}), 7.32(\mathrm{~d}, J=3.3$ $\mathrm{Hz}, 1 \mathrm{H}), 7.01(\mathrm{dd}, J=8.5,1.7 \mathrm{~Hz}, 1 \mathrm{H}), 6.48(\mathrm{dd}, J=3.3,0.8 \mathrm{~Hz}, 1 \mathrm{H}), 5.18-5.15(\mathrm{~m}, 1 \mathrm{H}), 5.14$ (s, 1H), $3.05(\mathrm{ddd}, J=14.7,7.3,1.0 \mathrm{~Hz}, 1 \mathrm{H}), 2.68(\mathrm{ddd}, J=14.7,6.8,1.0 \mathrm{~Hz}, 1 \mathrm{H}), 2.61(\mathrm{dtd}, J=$ 10.2, 7.0, 3.3 Hz, 1H), 2.39 (s, 3H), $2.21-2.09$ (m, 2H), 1.91 (ddt, J = 10.7, 6.9, $3.5 \mathrm{~Hz}, 1 \mathrm{H})$, $1.76(\mathrm{ddt}, J=14.1,9.4,4.9 \mathrm{~Hz}, 1 \mathrm{H}), 1.58(\mathrm{dd}, J=12.1,8.4 \mathrm{~Hz}, 3 \mathrm{H}), 1.45$ (ddd, $J=7.1,5.2,2.6$ $\mathrm{Hz}, 3 \mathrm{H}), 1.29$ (ddd, $J=14.6,7.9,2.7 \mathrm{~Hz}, 1 \mathrm{H}), 1.12-1.03(\mathrm{~m}, 1 \mathrm{H}) .{ }^{13} \mathrm{C} \mathrm{NMR}(101 \mathrm{MHz}$, $\left.\mathrm{CD}_{3} \mathrm{C}(\mathrm{O}) \mathrm{CD}_{3}\right) \delta 217.2,144.2,135.2,130.6,129.9,127.5,124.5,121.4,111.9,108.0,103.4,47.7$, 42.9, 37.9, 32.8, 28.3, 25.7, 25.5, 25.4, 21.4. HRMS (ESI) m/z: $[\mathrm{M}+\mathrm{H}]^{+}$Calcd for $\mathrm{C}_{20} \mathrm{H}_{26} \mathrm{NO}$ 296.2014, found 296.2023.

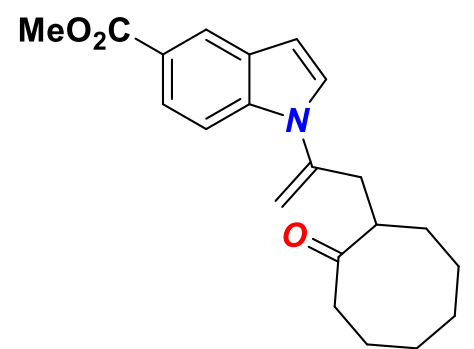

$1 \mathrm{~g}$
Methyl 1-(3-(2-oxocyclooctyl)prop-1-en-2-yl)-1H-indole-5-

carboxylate (1g): $1.0 \mathrm{mmol}$ scale, the product was purified through chromatography (toluene/DCM 50:1-3:1) afford as colourless oil. Yield 59\% (200 mg). ${ }^{1} \mathrm{H}$ NMR (400 MHz, $\left.\mathrm{CD}_{2} \mathrm{Cl}_{2}\right) \delta 8.39(\mathrm{~d}, J=$ $1.0 \mathrm{~Hz}, 1 \mathrm{H}), 7.91$ (dd, $J=8.7,1.7 \mathrm{~Hz}, 1 \mathrm{H}), 7.57$ (d, $J=8.8 \mathrm{~Hz}, 1 \mathrm{H})$, $7.29(\mathrm{~d}, J=3.3 \mathrm{~Hz}, 1 \mathrm{H}), 6.72(\mathrm{dd}, J=3.3,0.9 \mathrm{~Hz}, 1 \mathrm{H}), 5.27(\mathrm{~s}, 1 \mathrm{H})$,

$5.26(\mathrm{~s}, 1 \mathrm{H}), 3.97-3.88(\mathrm{~m}, 3 \mathrm{H}), 3.09(\mathrm{dd}, J=14.7,7.3 \mathrm{~Hz}, 1 \mathrm{H}), 2.68-2.55(\mathrm{~m}, 2 \mathrm{H}), 2.25-$ $2.11(\mathrm{~m}, 2 \mathrm{H}), 1.91(\mathrm{ddt}, J=10.9,7.4,3.4 \mathrm{~Hz}, 1 \mathrm{H}), 1.79$ (ddd, $J=9.9,5.8,3.6 \mathrm{~Hz}, 1 \mathrm{H}), 1.68-$ $1.60(\mathrm{~m}, 3 \mathrm{H}), 1.55-1.44(\mathrm{~m}, 3 \mathrm{H}), 1.38-1.31(\mathrm{~m}, 1 \mathrm{H}), 1.14-1.04(\mathrm{~m}, 1 \mathrm{H}) .{ }^{13} \mathrm{C}$ NMR $(101$ $\left.\mathrm{MHz}, \mathrm{CD}_{2} \mathrm{Cl}_{2}\right) \delta 217.4,167.6,142.7,138.2,128.6,128.0,123.6,123.3,122.3,110.8,109.8$, 104.2, 51.7, 47.1, 42.3, 37.2, 32.2, 27.5, 25.0, 25.0, 24.6. HRMS (ESI) m/z: $[\mathrm{M}+\mathrm{H}]^{+}$Calcd for $\mathrm{C}_{21} \mathrm{H}_{26} \mathrm{NO}_{3} 340.1913$, found 340.1901 .

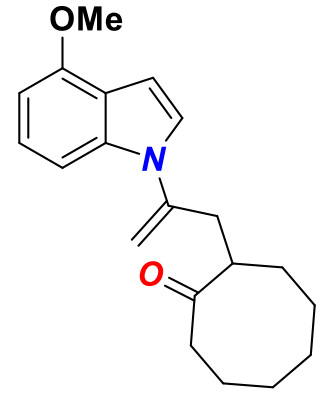

$1 \mathrm{~h}$

2-(2-(4-methoxy-1H-indol-1-yl)allyl)cyclooctan-1-one (1h): $1.0 \mathrm{mmol}$ scale, the product was purified through chromatography (toluene/DCM 50:1-5:1) afford as colourless oil. Yield 76\% (236 mg). ${ }^{1} \mathrm{H}$ NMR (400 MHz, $\left.\mathrm{CD}_{2} \mathrm{Cl}_{2}\right) \delta 7.13(\mathrm{~d}, J=5.9 \mathrm{~Hz}, 2 \mathrm{H}), 7.07(\mathrm{~d}, J=3.0 \mathrm{~Hz}, 1 \mathrm{H}), 6.64(\mathrm{~d}, J=3.3$ $\mathrm{Hz}, 1 \mathrm{H}), 6.55$ (d, $J=7.0 \mathrm{~Hz}, 1 \mathrm{H}), 5.18(\mathrm{~d}, J=1.1 \mathrm{~Hz}, 1 \mathrm{H}), 5.15(\mathrm{~s}, 1 \mathrm{H})$, $3.94(\mathrm{~s}, 3 \mathrm{H}), 3.05(\mathrm{dd}, J=14.2,6.7 \mathrm{~Hz}, 1 \mathrm{H}), 2.67-2.52(\mathrm{~m}, 2 \mathrm{H}), 2.16(\mathrm{t}, J$ $=6.6 \mathrm{~Hz}, 2 \mathrm{H}), 1.87(\mathrm{ddd}, J=14.1,6.9,3.3 \mathrm{~Hz}, 1 \mathrm{H}), 1.77(\mathrm{dt}, J=16.0,6.0$ $\mathrm{Hz}, 1 \mathrm{H}), 1.60(\mathrm{dq}, J=9.7,4.8 \mathrm{~Hz}, 3 \mathrm{H}), 1.45(\mathrm{dq}, J=9.3,4.6,3.9 \mathrm{~Hz}, 3 \mathrm{H}), 1.31-1.28(\mathrm{~m}, 1 \mathrm{H})$, 
$1.06(\mathrm{dd}, J=13.4,5.2 \mathrm{~Hz}, 1 \mathrm{H}) .{ }^{13} \mathrm{C}$ NMR $\left(101 \mathrm{MHz}, \mathrm{CD}_{2} \mathrm{Cl}_{2}\right) \delta 217.7,153.4,143.2,137.2$, 125.0, 123.0, 119.6, 108.8, 104.7, 100.2, 100.0, 55.3, 47.1, 42.4, 37.3, 32.1, 27.6, 25.1, 25.0, 24.7. HRMS (ESI) m/z: $[\mathrm{M}+\mathrm{H}]^{+}$Calcd for $\mathrm{C}_{20} \mathrm{H}_{26} \mathrm{NO}_{2} 312.1964$, found 312.1958 .

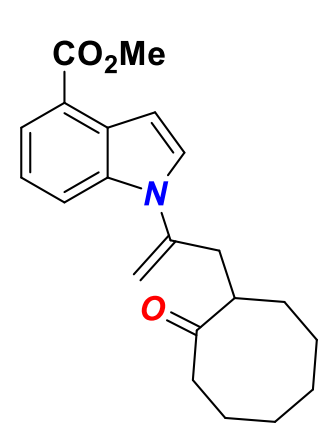

$1 \mathrm{i}$

Methyl 1-(3-(2-oxocyclooctyl)prop-1-en-2-yl)-1H-indole-4-carboxylate (1i): $1.0 \mathrm{mmol}$ scale, the product was purified through chromatography (toluene/DCM 50:1-3:1) afford as colourless oil. Yield 76\% (236 mg). ${ }^{1} \mathrm{H}$ NMR $\left(400 \mathrm{MHz}, \mathrm{CD}_{3} \mathrm{C}(\mathrm{O}) \mathrm{CD}_{3}\right) \delta 7.86(\mathrm{dd}, J=7.5,0.9 \mathrm{~Hz}, 1 \mathrm{H}), 7.81(\mathrm{~d}, J$ $=8.2 \mathrm{~Hz}, 1 \mathrm{H}), 7.55(\mathrm{~d}, J=3.3 \mathrm{~Hz}, 1 \mathrm{H}), 7.29(\mathrm{t}, J=7.9 \mathrm{~Hz}, 1 \mathrm{H}), 7.21(\mathrm{dd}, J$ $=3.3,0.8 \mathrm{~Hz}, 1 \mathrm{H}), 5.30(\mathrm{~s}, 1 \mathrm{H}), 5.21(\mathrm{~s}, 1 \mathrm{H}), 3.94(\mathrm{~s}, 3 \mathrm{H}), 3.07(\mathrm{ddd}, J=$ $15.1,7.8,1.0 \mathrm{~Hz}, 1 \mathrm{H}), 2.71$ (ddd, $J=15.1,6.6,1.0 \mathrm{~Hz}, 1 \mathrm{H}), 2.65-2.55(\mathrm{~m}$, $1 \mathrm{H}), 2.16(\mathrm{dd}, J=7.6,4.9 \mathrm{~Hz}, 2 \mathrm{H}), 1.96-1.87(\mathrm{~m}, 1 \mathrm{H}), 1.84-1.70(\mathrm{~m}, 1 \mathrm{H}), 1.65-1.54(\mathrm{~m}$, $3 \mathrm{H}), 1.50-1.40(\mathrm{~m}, 3 \mathrm{H}), 1.34-1.26(\mathrm{~m}, 1 \mathrm{H}), 1.13-1.02(\mathrm{~m}, 1 \mathrm{H}) .{ }^{13} \mathrm{C}$ NMR (101 MHz, $\left.\mathrm{CD}_{3} \mathrm{C}(\mathrm{O}) \mathrm{CD}_{3}\right) \delta 217.1,167.9,143.8,137.6,129.8,124.2,122.6,122.2,116.9,110.2,104.8,51.9$, 47.8, 42.8, 38.1, 32.9, 28.2, 25.7, 25.6, 25.4. HRMS (ESI) m/z: $[\mathrm{M}+\mathrm{H}]^{+}$Calcd for $\mathrm{C}_{21} \mathrm{H}_{26} \mathrm{NO}_{3}$ 340.1913 , found 340.1925 .

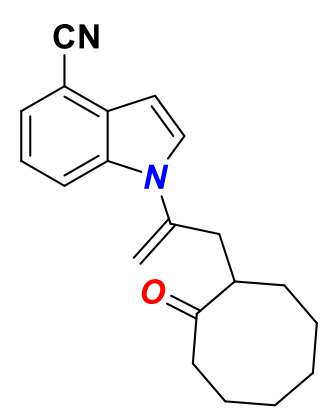

1j $\quad 1.0 \mathrm{~Hz}, 1 \mathrm{H}), 2.76-2.70(\mathrm{~m}, 1 \mathrm{H}), 2.66-2.57(\mathrm{~m}, 1 \mathrm{H}), 2.24-2.14(\mathrm{~m}, 2 \mathrm{H})$, $1.92(\mathrm{ddd}, J=10.7,7.1,3.6 \mathrm{~Hz}, 1 \mathrm{H}), 1.79(\mathrm{dt}, J=13.3,6.5 \mathrm{~Hz}, 1 \mathrm{H}), 1.68-1.55(\mathrm{~m}, 3 \mathrm{H}), 1.46$ $(\mathrm{dt}, J=5.0,1.9 \mathrm{~Hz}, 3 \mathrm{H}), 1.37-1.27(\mathrm{~m}, 1 \mathrm{H}), 1.14-1.01(\mathrm{~m}, 1 \mathrm{H}) .{ }^{13} \mathrm{C}$ NMR $(101 \mathrm{MHz}$, $\left.\mathrm{CD}_{3} \mathrm{C}(\mathrm{O}) \mathrm{CD}_{3}\right) \delta 217.0,143.4,136.4,130.9,130.8,126.1,122.8,118.7,117.1,110.9,103.8$, 101.9, 47.9, 42.6, 37.8, 32.7, 28.1, 25.7, 25.6, 25.3. HRMS (ESI) $\mathrm{m} / \mathrm{z}:[\mathrm{M}+\mathrm{H}]^{+}$Calcd for $\mathrm{C}_{20} \mathrm{H}_{23} \mathrm{~N}_{2} \mathrm{O} 307.1810$, found 307.1824 . 


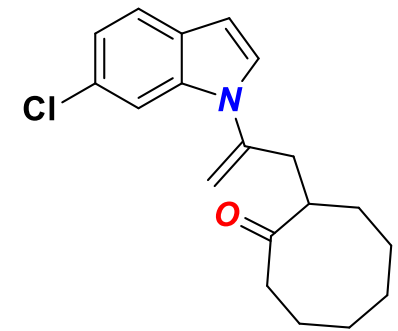

$1 \mathrm{k}$

2-(2-(6-Chloro-1H-indol-1-yl)allyl)cyclooctan-1-one (1k): $1.0 \mathrm{mmol}$ scale, the product was purified through chromatography (toluene/DCM 50:1-10:1) afford as colourless oil. Yield 76\% (236 mg). ${ }^{1} \mathrm{H}$ NMR (400 $\left.\mathrm{MHz}, \mathrm{CD}_{3} \mathrm{C}(\mathrm{O}) \mathrm{CD}_{3}\right) \delta 7.59(\mathrm{~d}, J=8.4 \mathrm{~Hz}, 1 \mathrm{H}), 7.55(\mathrm{~d}, J=1.7 \mathrm{~Hz}, 1 \mathrm{H})$, $7.43(\mathrm{~d}, J=3.3 \mathrm{~Hz}, 1 \mathrm{H}), 7.09(\mathrm{dd}, J=8.4,1.8 \mathrm{~Hz}, 1 \mathrm{H}), 6.61(\mathrm{~d}, J=3.4$ $\mathrm{Hz}, 1 \mathrm{H}), 5.28(\mathrm{~s}, 1 \mathrm{H}), 5.21(\mathrm{~s}, 1 \mathrm{H}), 3.04(\mathrm{dd}, J=14.9,7.5 \mathrm{~Hz}, 1 \mathrm{H}), 2.69$ (dd, $J=14.9,6.6 \mathrm{~Hz}, 1 \mathrm{H}), 2.62(\mathrm{dtd}, J=10.3,7.1,3.4 \mathrm{~Hz}, 1 \mathrm{H}), 2.19$ (dd, $J=7.6,5.0 \mathrm{~Hz}, 2 \mathrm{H}), 1.93(\mathrm{ddt}, J=14.2,6.7,4.0 \mathrm{~Hz}, 1 \mathrm{H}), 1.81(\mathrm{dq}, J=12.9,6.6 \mathrm{~Hz}, 1 \mathrm{H}), 1.65-$ $1.55(\mathrm{~m}, 3 \mathrm{H}), 1.46(\mathrm{dd}, J=7.0,4.2 \mathrm{~Hz}, 3 \mathrm{H}), 1.34(\mathrm{dd}, J=12.9,8.7 \mathrm{~Hz}, 1 \mathrm{H}), 1.14-1.04(\mathrm{~m}, 1 \mathrm{H})$. ${ }^{13} \mathrm{C}$ NMR $\left(101 \mathrm{MHz}, \mathrm{CD}_{3} \mathrm{C}(\mathrm{O}) \mathrm{CD}_{3}\right) \delta 217.1,143.8,137.1,128.8,128.7,128.5,122.8,121.4$, 111.9, 109.7, 104.0, 47.9, 42.7, 37.9, 32.8, 28.2, 25.8, 25.7, 25.41. HRMS (ESI) m/z: $[\mathrm{M}+\mathrm{H}]^{+}$ Calcd for $\mathrm{C}_{19} \mathrm{H}_{23} \mathrm{ClNO} 316.1468$, found 316.1460.

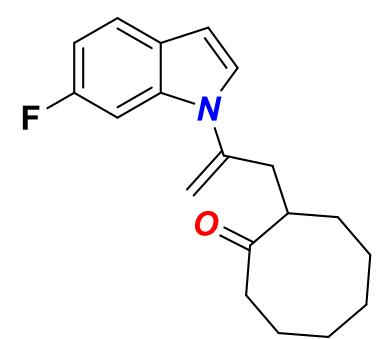

11

2-(2-(7-Fluoro-1H-indol-1-yl)allyl)cyclooctan-1-one (11): $1.0 \mathrm{mmol}$ scale, the product was purified through chromatography (toluene/DCM 50:1-10:1) afford as colourless oil. Yield 76\% (236 mg). ${ }^{1} \mathrm{H}$ NMR (400 $\left.\mathrm{MHz}, \mathrm{CD}_{3} \mathrm{C}(\mathrm{O}) \mathrm{CD}_{3}\right) \delta 7.58(\mathrm{dd}, J=8.7,5.5 \mathrm{~Hz}, 1 \mathrm{H}), 7.39(\mathrm{~d}, J=3.4 \mathrm{~Hz}$, $1 \mathrm{H}), 7.27$ (dd, $J=10.5,2.4 \mathrm{~Hz}, 1 \mathrm{H}), 6.90(\mathrm{ddd}, J=9.6,8.6,2.3 \mathrm{~Hz}, 1 \mathrm{H})$, $6.60(\mathrm{dd}, J=3.4,0.9 \mathrm{~Hz}, 1 \mathrm{H}), 5.24(\mathrm{t}, J=1.1 \mathrm{~Hz}, 1 \mathrm{H}), 5.19$ (s, $1 \mathrm{H}), 3.04$ (ddd, $J=14.8,7.5,1.0 \mathrm{~Hz}, 1 \mathrm{H}$ ), 2.69 (ddd, $J=14.8,6.6,1.0 \mathrm{~Hz}, 1 \mathrm{H}$ ), $2.62(\mathrm{dtd}, J=10.1,6.8,3.3 \mathrm{~Hz}, 1 \mathrm{H}), 2.24-2.12(\mathrm{~m}, 2 \mathrm{H}), 1.98-1.88(\mathrm{~m}, 1 \mathrm{H}), 1.85-1.73(\mathrm{~m}$, $1 \mathrm{H}), 1.67-1.55(\mathrm{~m}, 3 \mathrm{H}), 1.52-1.42(\mathrm{~m}, 3 \mathrm{H}), 1.38-1.28(\mathrm{~m}, 1 \mathrm{H}), 1.15-1.03(\mathrm{~m}, 1 \mathrm{H}) .{ }^{13} \mathrm{C}$ NMR $\left(101 \mathrm{MHz}, \mathrm{CD}_{3} \mathrm{C}(\mathrm{O}) \mathrm{CD}_{3}\right) \delta 217.1,162.0,159.6,143.9,136.8,136.6,128.3,128.2,126.7$, 122.7, 122.6, 109.4, 109.2, 109.2, 104.0, 98.6, 98.3, 47.8, 42.7, 37.8, 32.8, 28.2, 25.7, 25.6, 25.4. ${ }^{19} \mathrm{~F}$ NMR $\left(376 \mathrm{MHz}, \mathrm{CD}_{3} \mathrm{C}(\mathrm{O}) \mathrm{CD}_{3}\right) \delta$-121.84. HRMS (ESI) m/z: $[\mathrm{M}+\mathrm{H}]^{+}$Calcd for $\mathrm{C}_{19} \mathrm{H}_{22} \mathrm{FNO} 300.1764$, found 300.1789 .

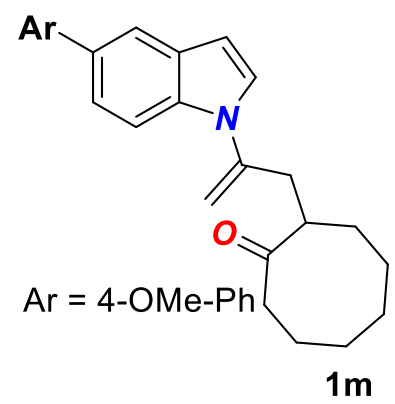

\section{2-(2-(5-(4-Methoxyphenyl)-1H-indol-1-yl)allyl)cyclooctan-1-one}

(1m): $1.0 \mathrm{mmol}$ scale, the product was purified through chromatography (toluene/DCM 50:1-5:1) afford as colourless oil. Yield $70 \%(271 \mathrm{mg}) .{ }^{1} \mathrm{H}$ NMR (400 MHz, $\left.\mathrm{CD}_{2} \mathrm{Cl}_{2}\right) \delta 7.82(\mathrm{~s}, 1 \mathrm{H}), 7.62$ (t, $J=$ 
$8.4 \mathrm{~Hz}, 3 \mathrm{H}), 7.47(\mathrm{dd}, J=8.6,1.6 \mathrm{~Hz}, 1 \mathrm{H}), 7.25(\mathrm{~d}, J=3.3 \mathrm{~Hz}, 1 \mathrm{H}), 7.03(\mathrm{~d}, J=8.7 \mathrm{~Hz}, 2 \mathrm{H})$, $6.66(\mathrm{~d}, J=3.2 \mathrm{~Hz}, 1 \mathrm{H}), 5.25(\mathrm{~s}, 1 \mathrm{H}), 5.21(\mathrm{~s}, 1 \mathrm{H}), 3.88(\mathrm{~s}, 3 \mathrm{H}), 3.16-3.08(\mathrm{~m}, 1 \mathrm{H}), 2.71-2.60$ (m, 2H), $2.27-2.20(\mathrm{~m}, 2 \mathrm{H}), 1.95(\mathrm{ddt}, J=10.8,7.2,3.8 \mathrm{~Hz}, 1 \mathrm{H}), 1.83(\mathrm{dt}, J=15.4,6.0 \mathrm{~Hz}$, $1 \mathrm{H}), 1.70-1.63(\mathrm{~m}, 3 \mathrm{H}), 1.56-1.46(\mathrm{~m}, 3 \mathrm{H}), 1.37(\mathrm{dq}, J=8.8,2.4 \mathrm{~Hz}, 1 \mathrm{H}), 1.16-1.08(\mathrm{~m}$, 1H). ${ }^{13} \mathrm{C}$ NMR $\left(101 \mathrm{MHz}, \mathrm{CD}_{2} \mathrm{Cl}_{2}\right) \delta 217.7,158.7,143.0,135.1,134.6,133.2,129.8,128.1$, 127.1, 121.6, 118.7, 114.1, 111.6, 108.4, 103.4, 55.3, 47.3, 42.3, 37.2, 32.0, 27.6, 25.3, 25.1, 24.7. HRMS (ESI) m/z: [M+H] ${ }^{+}$Calcd for $\mathrm{C}_{26} \mathrm{H}_{30} \mathrm{NO}_{2} 388.2277$, found 388.2293.

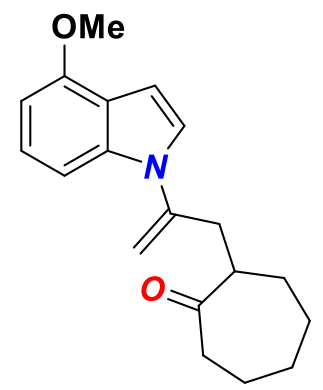

$1 \mathrm{n}$

2-(2-(4-Methoxy-1H-indol-1-yl)allyl)cycloheptan-1-one (1n): $1.0 \mathrm{mmol}$ scale, the product was purified through chromatography (toluene/DCM 50:15:1) afford as colourless oil. Yield 78\% (232 mg). ${ }^{1} \mathrm{H}$ NMR (400 MHz, $\left.\mathrm{CD}_{3} \mathrm{C}(\mathrm{O}) \mathrm{CD}_{3}\right) \delta 7.23(\mathrm{~d}, J=3.4 \mathrm{~Hz}, 1 \mathrm{H}), 7.16-7.10(\mathrm{~m}, 2 \mathrm{H}), 6.62(\mathrm{dd}, J=$ 3.3, $0.7 \mathrm{~Hz}, 1 \mathrm{H}), 6.57(\mathrm{dd}, J=7.4,1.1 \mathrm{~Hz}, 1 \mathrm{H}), 5.22$ (t, $J=1.1 \mathrm{~Hz}, 1 \mathrm{H}), 5.19$ (s, 1H), 3.92 (s, 3H), 3.12 (ddd, $J=15.1,5.7,1.2 \mathrm{~Hz}, 1 \mathrm{H}), 2.53$ (ddd, $J=$ 15.1, 8.4, $0.8 \mathrm{~Hz}, 1 \mathrm{H}), 2.46-2.36(\mathrm{~m}, 1 \mathrm{H}), 2.35-2.27$ (m, 2H), 1.84 (dddd, $J=11.9,5.4,3.4,1.4 \mathrm{~Hz}, 1 \mathrm{H}), 1.69$ (tdd, $J=12.9,4.4,2.0 \mathrm{~Hz}, 3 \mathrm{H}), 1.43$ (dddt, $J=18.9,13.1$, 7.8, 3.0 Hz, 1H), 1.36 - $1.21(\mathrm{~m}, 3 \mathrm{H}) .{ }^{13} \mathrm{C} \mathrm{NMR}\left(101 \mathrm{MHz}, \mathrm{CD}_{3} \mathrm{C}(\mathrm{O}) \mathrm{CD}_{3}\right) \delta 213.3,154.3,144.4$, $138.1,125.9,123.9,120.6,109.5,105.3,101.0,100.9,55.5,49.3,43.4,37.9,30.8,29.9,29.2$, 24.6. HRMS (ESI) m/z: $[\mathrm{M}+\mathrm{H}]^{+}$Calcd for $\mathrm{C}_{19} \mathrm{H}_{24} \mathrm{NO}_{2} 298.1807$, found 298.1831.

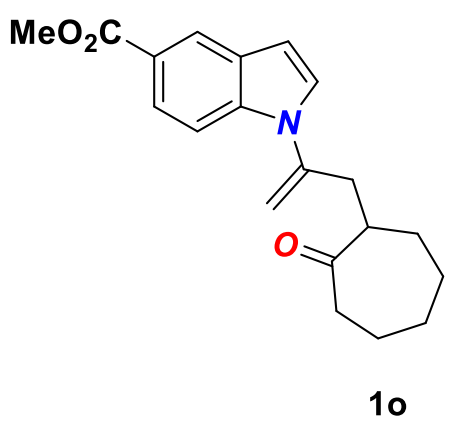

Methyl 1-(3-(2-oxocycloheptyl)prop-1-en-2-yl)-1H-indole-5carboxylate (10): $1.0 \mathrm{mmol}$ scale, the product was purified through chromatography (toluene/DCM 50:1-3:1) afford as colourless oil. Yield 67\% (218 mg). ${ }^{1} \mathrm{H}$ NMR (400 MHz, $\left.\mathrm{CD}_{3} \mathrm{C}(\mathrm{O}) \mathrm{CD}_{3}\right) \delta 8.35$ (td, $J=2.3,1.8,0.9 \mathrm{~Hz}, 1 \mathrm{H}), 7.88(\mathrm{dd}, J=8.8,1.7 \mathrm{~Hz}, 1 \mathrm{H}), 7.62(\mathrm{dt}, J=$ $8.8,0.8 \mathrm{~Hz}, 1 \mathrm{H}), 7.51(\mathrm{~d}, J=3.4 \mathrm{~Hz}, 1 \mathrm{H}), 6.76(\mathrm{dd}, J=3.3,0.8 \mathrm{~Hz}$, $1 \mathrm{H}), 5.35(\mathrm{t}, J=1.0 \mathrm{~Hz}, 1 \mathrm{H}), 5.28(\mathrm{~s}, 1 \mathrm{H}), 3.90(\mathrm{~s}, 3 \mathrm{H}), 3.14$ (ddd, $J$ $=15.3,6.1,1.3 \mathrm{~Hz}, 1 \mathrm{H}), 2.60(\mathrm{ddd}, J=15.3,8.1,0.9 \mathrm{~Hz}, 1 \mathrm{H}), 2.44(\mathrm{dddd}, J=9.8,8.1,6.1,3.4$ $\mathrm{Hz}, 1 \mathrm{H}), 2.38-2.29(\mathrm{~m}, 2 \mathrm{H}), 1.92-1.82(\mathrm{~m}, 1 \mathrm{H}), 1.82-1.65(\mathrm{~m}, 3 \mathrm{H}), 1.51-1.39(\mathrm{~m}, 1 \mathrm{H})$, $1.38-1.19(\mathrm{~m}, 3 \mathrm{H}) .{ }^{13} \mathrm{C} \mathrm{NMR}\left(101 \mathrm{MHz}, \mathrm{CD}_{3} \mathrm{C}(\mathrm{O}) \mathrm{CD}_{3}\right) \delta$ 213.3, 168.0, 144.0, 139.2, 129.6, 
129.4, 124.3, 124.0, 123.1, 111.8, 110.8, 105.0, 52.0, 49.3, 43.5, 37.9, 31.0, 29.9, 29.3, 24.6. HRMS (ESI) m/z: [M+ H] ${ }^{+}$Calcd for $\mathrm{C}_{20} \mathrm{H}_{24} \mathrm{NO}_{3} 326.1756$, found 326.1748 .

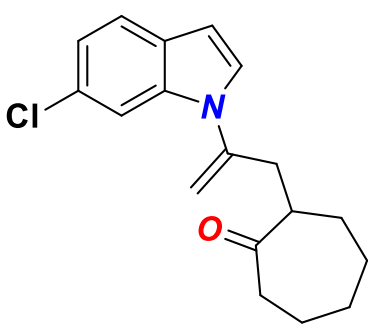

1p $=3.3,0.9 \mathrm{~Hz}, 1 \mathrm{H}), 5.30(\mathrm{~d}, J=1.2 \mathrm{~Hz}, 1 \mathrm{H}), 5.24(\mathrm{~s}, 1 \mathrm{H}), 3.09$ (ddd, $J=$ scale, the product was purified through chromatography (toluene/DCM 50:1-10:1) afford as colourless oil. Yield 75\% (226 mg). ${ }^{1} \mathrm{H}$ NMR (400 $\left.\mathrm{MHz}, \mathrm{CD}_{3} \mathrm{C}(\mathrm{O}) \mathrm{CD}_{3}\right) \delta 7.59(\mathrm{dd}, J=8.3,0.5 \mathrm{~Hz}, 1 \mathrm{H}), 7.55(\mathrm{~d}, J=2.0 \mathrm{~Hz}$, $1 \mathrm{H}), 7.42(\mathrm{~d}, J=3.4 \mathrm{~Hz}, 1 \mathrm{H}), 7.08$ (dd, $J=8.4,1.9 \mathrm{~Hz}, 1 \mathrm{H}), 6.61$ (dd, $J$

2-(2-(6-Chloro-1H-indol-1-yl)allyl)cycloheptan-1-one (1p): $1.0 \mathrm{mmol}$ 15.2, 6.1, $1.3 \mathrm{~Hz}, 1 \mathrm{H}$ ), 2.57 (ddd, $J=15.2,8.1,0.9 \mathrm{~Hz}, 1 \mathrm{H}$ ), 2.44 (dddd, $J=9.8,8.1,6.1,3.4 \mathrm{~Hz}$, $1 \mathrm{H}), 2.35(\mathrm{dd}, J=7.8,4.8 \mathrm{~Hz}, 2 \mathrm{H}), 1.91-1.82(\mathrm{~m}, 1 \mathrm{H}), 1.79-1.65(\mathrm{~m}, 3 \mathrm{H}), 1.57-1.43(\mathrm{~m}$, $1 \mathrm{H}), 1.38-1.24(\mathrm{~m}, 3 \mathrm{H}) .{ }^{13} \mathrm{C} \mathrm{NMR}\left(101 \mathrm{MHz}, \mathrm{CD}_{3} \mathrm{C}(\mathrm{O}) \mathrm{CD}_{3}\right) \delta 213.3,144.0,137.2,128.8$, 128.6, 128.4, 122.8, 121.3, 111.8, 110.3, 103.9, 49.4, 43.4, 37.9, 31.0, 30.0, 29.2, 24.6. HRMS (ESI) $\mathrm{m} / \mathrm{z}:[\mathrm{M}+\mathrm{H}]^{+}$Calcd for $\mathrm{C}_{18} \mathrm{H}_{21} \mathrm{ClNO} 302.1312$, found 301.1329 .

\section{Cycloisomerization of $N$-vinyl indoles using Cobaloxime/H2 system}

General procedure for the cycloisomerization of $\mathrm{N}$-vinyl-indoles using Cobaloxime/ $\mathrm{H}_{2}$ system

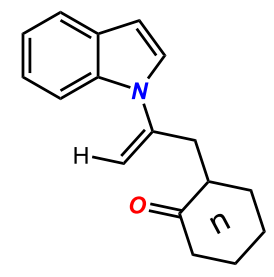

1

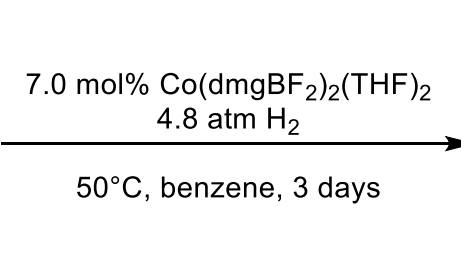

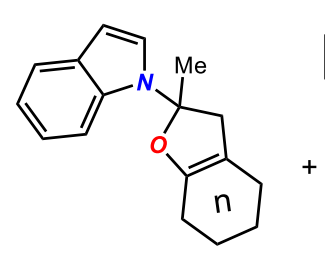

2

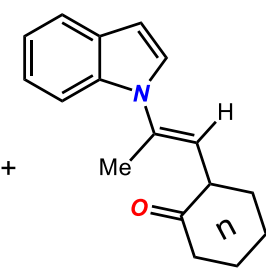

3

The cobalt or chromium catalysts and $N$-vinyl indole $(0.2 \mathrm{mmol})$ were weighed in a glass vial and transferred to a Fisher-Porter tube equipped with a magnetic stir-bar using $4 \mathrm{~mL}$ of dry and degassed benzene. The tube was then placed behind an appropriate blast shield before it was pressurized to $2.0 \mathrm{~atm}$ of $\mathrm{H}_{2}$. The vessel was then immediately vented and repressurized and this operation was repeated twenty times, to push out the inert atmosphere. The vessel was pressurized to $2.0 \mathrm{~atm}$ of $\mathrm{H}_{2}$ for 30 seconds. If no change in pressure was observed, the tube was pressurized to 4.8 atm of $\mathrm{H}_{2}$, and stirred at $60{ }^{\circ} \mathrm{C}$ for 3 days. (NOTE: The pressurized Fisher- 
Porter tube should always be placed behind an appropriate blast shield!!!) After the indicated time, the Fisher-Porter tube was cooled to room temperature, $\mathrm{H}_{2}$ gas was vented slowly, then the apparatus was disassembled. The crude reaction mixture was filtered through a short silica gel plug, and the plug was washed with ethyl acetate. The resulting filtrate was concentrated and the crude reaction mixture was analysed by ${ }^{1} \mathrm{H}$ NMR $\left(\mathrm{CDCl}_{3}, \mathrm{CD}_{2} \mathrm{Cl}_{2}, \mathrm{C}_{6} \mathrm{D}_{6}\right.$ or $\left.\mathrm{CD}_{3} \mathrm{C}(\mathrm{O}) \mathrm{CD}_{3}\right)$ to determine product distribution and selectivity. Unless stated otherwise, the crude product was purified by chromatography on silica gel, concentrated under reduced pressure and stored neat or as a solution in acetone.

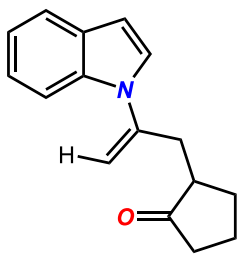

$1 \mathrm{~b}$

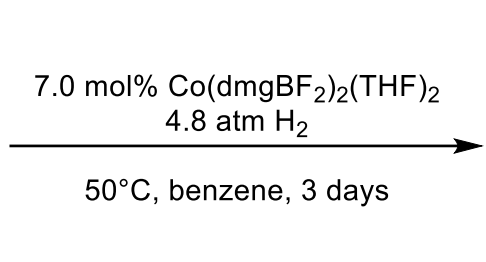$$
\text { . }
$$

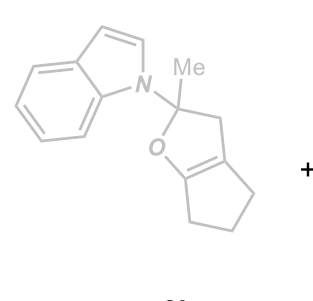

$2 \mathbf{b}$

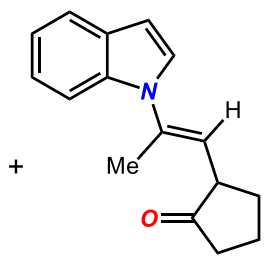

3b

\section{(E)-2-(2-(1H-indol-1-yl)prop-1-en-1-yl)cyclopentan-1-one (3b)}

According to general process, $\mathbf{1 b}(0.2 \mathrm{mmol})$ was subjected to the reaction condition. The product was purified through chromatography (hexane / EtOAc $=10: 1$ ) afford $\mathbf{3 b}$ as a colorless oil. Yield 65\% (31.1 mg). ${ }^{1} \mathrm{H}$ NMR (400 MHz, $\left.\mathrm{CD}_{3} \mathrm{C}(\mathrm{O}) \mathrm{CD}_{3}\right) \delta 7.56(\mathrm{~d}, J=9.1 \mathrm{~Hz}, 2 \mathrm{H}), 7.34(\mathrm{~d}$, $J=3.3 \mathrm{~Hz}, 1 \mathrm{H}$ ), 7.15 (ddd, $J=8.1,7.0,1.3 \mathrm{~Hz}, 1 \mathrm{H}), 7.05$ (ddd, $J=8.1,7.0,1.0 \mathrm{~Hz}, 1 \mathrm{H}), 6.53$ (dd, $J=3.3,0.8 \mathrm{~Hz}, 1 \mathrm{H}), 5.49$ (dd, $J=8.8,1.2 \mathrm{~Hz}, 1 \mathrm{H}), 3.36-3.26(\mathrm{~m}, 1 \mathrm{H}), 2.48-2.39(\mathrm{~m}, 1 \mathrm{H})$, $2.38-2.29(\mathrm{~m}, 1 \mathrm{H}), 2.23(\mathrm{~d}, J=1.3 \mathrm{~Hz}, 3 \mathrm{H}), 2.22-2.15(\mathrm{~m}, 1 \mathrm{H}), 2.15-2.08(\mathrm{~m}, 1 \mathrm{H}), 2.01-$ $1.91(\mathrm{~m}, 1 \mathrm{H}), 1.91-1.79(\mathrm{~m}, 1 \mathrm{H}) .{ }^{13} \mathrm{C}$ NMR $\left(125 \mathrm{MHz}, \mathrm{CD}_{3} \mathrm{C}(\mathrm{O}) \mathrm{CD}_{3}\right) \delta$ 216.6, 136.1, 136.0, 129.3, 126.9, 121.8, 121.7, 120.8, 119.8, 111.41, 102.4, 48.9, 37.0, 30.7, 20.9, 16.9. HRMS (ESI) $\mathrm{m} / \mathrm{z}:[\mathrm{M}+\mathrm{H}]^{+}$Calcd for $\mathrm{C}_{16} \mathrm{H}_{18} \mathrm{NO} 240.1388$, found 240.1409 .

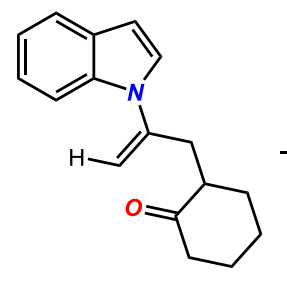

$1 \mathbf{a}$

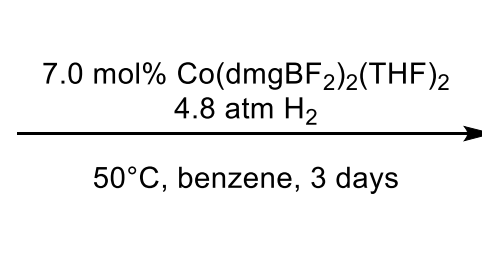

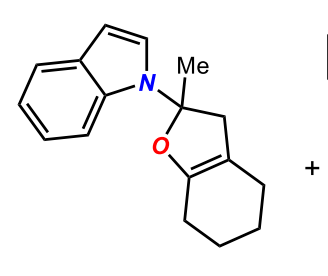

$2 a$

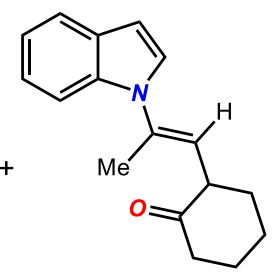

$3 a$

According to general process, 1a $(0.2 \mathrm{mmol})$ was subjected to the reaction condition. The product was purified through chromatography (hexane / EtOAc $=10: 1$ ) afford: $2 \mathbf{a}$ as a colorless oil. Yield 40\% (20.2 mg). 3a was isolated as a colorless oil. Yield 45\% (22.8 mg). 


\section{1-(2-Methyl-2,3,4,5,6,7-hexahydrobenzofuran-2-yl)-1H-indole (2a)}

${ }^{1} \mathrm{H}$ NMR $\left(500 \mathrm{MHz}, \mathrm{CDCl}_{3}\right) \delta 7.63(\mathrm{~d}, J=7.7 \mathrm{~Hz}, 1 \mathrm{H}), 7.43-7.38(\mathrm{~m}, 2 \mathrm{H}), 7.20-7.16(\mathrm{~m}, 1 \mathrm{H})$, $7.15-7.10(\mathrm{~m}, 1 \mathrm{H}), 6.48$ (d, $J=3.4 \mathrm{~Hz}, 1 \mathrm{H}), 3.22$ (d, J = 15.2 Hz, 1H), 2.79 (d, $J=15.4 \mathrm{~Hz}$, $1 \mathrm{H}), 2.18(\mathrm{dt}, J=6.0,3.1 \mathrm{~Hz}, 2 \mathrm{H}), 2.11-2.03(\mathrm{~m}, 2 \mathrm{H}), 1.97(\mathrm{~s}, 3 \mathrm{H}), 1.86-1.77(\mathrm{~m}, 2 \mathrm{H}), 1.77-$ $1.68(\mathrm{~m}, 2 \mathrm{H}) .{ }^{13} \mathrm{C} \mathrm{NMR}\left(125 \mathrm{MHz}, \mathrm{CDCl}_{3}\right) \delta 149.6,134.3,130.3,124.8,121.7,121.2,119.8$, 112.1, 105.5, 101.4, 96.0, 46.0, 27.9, 23.3, 23.1, 23.0, 22.9. HRMS (ESI) m/z: $[\mathrm{M}+\mathrm{H}]^{+}$Calcd for $\mathrm{C}_{17} \mathrm{H}_{20} \mathrm{NO} 254.1545$, found 254.1554.

(E)-2-(2-(1H-indol-1-yl)prop-1-en-1-yl)cyclohexan-1-one (3a)

${ }^{1} \mathrm{H}$ NMR (400 MHz, $\left.\mathrm{CD}_{3} \mathrm{C}(\mathrm{O}) \mathrm{CD}_{3}\right) \delta 7.70(\mathrm{dd}, J=8.3,0.9 \mathrm{~Hz}, 1 \mathrm{H}), 7.56(\mathrm{~d}, J=7.8 \mathrm{~Hz}, 1 \mathrm{H})$, $7.34(\mathrm{~d}, J=3.3 \mathrm{~Hz}, 1 \mathrm{H}), 7.20-7.12(\mathrm{~m}, 1 \mathrm{H}), 7.05(\mathrm{ddd}, J=7.8,6.9,0.8 \mathrm{~Hz}, 1 \mathrm{H}), 6.53(\mathrm{dd}, J=$ 3.3, $0.9 \mathrm{~Hz}, 1 \mathrm{H}), 5.79(\mathrm{dd}, J=9.2,1.2 \mathrm{~Hz}, 1 \mathrm{H}), 3.64-3.53(\mathrm{~m}, 1 \mathrm{H}), 2.51(\mathrm{tdd}, J=12.6,5.8,1.0$ $\mathrm{Hz}, 1 \mathrm{H}), 2.43-2.36(\mathrm{~m}, 1 \mathrm{H}), 2.27-2.20(\mathrm{~m}, 1 \mathrm{H}), 2.16(\mathrm{~d}, J=1.2 \mathrm{~Hz}, 3 \mathrm{H}), 2.13-2.08(\mathrm{~m}, 1 \mathrm{H})$, $1.96-1.87(\mathrm{~m}, 2 \mathrm{H}), 1.79-1.66(\mathrm{~m}, 2 \mathrm{H}) .{ }^{13} \mathrm{C} \mathrm{NMR}\left(101 \mathrm{MHz}, \mathrm{CD}_{3} \mathrm{C}(\mathrm{O}) \mathrm{CD}_{3}\right) \delta 209.5,136.7$, $134.8,130.0$, 127.5, 122.9, 122.6, 121.5, 120.6, 112.3, 103.1, 50.5, 42.4, 35.6, 28.4, 25.3, 17.3. HRMS (ESI) m/z: [M + H $]^{+}$Calcd for $\mathrm{C}_{17} \mathrm{H}_{20} \mathrm{NO} 254.1545$, found 254.1559.
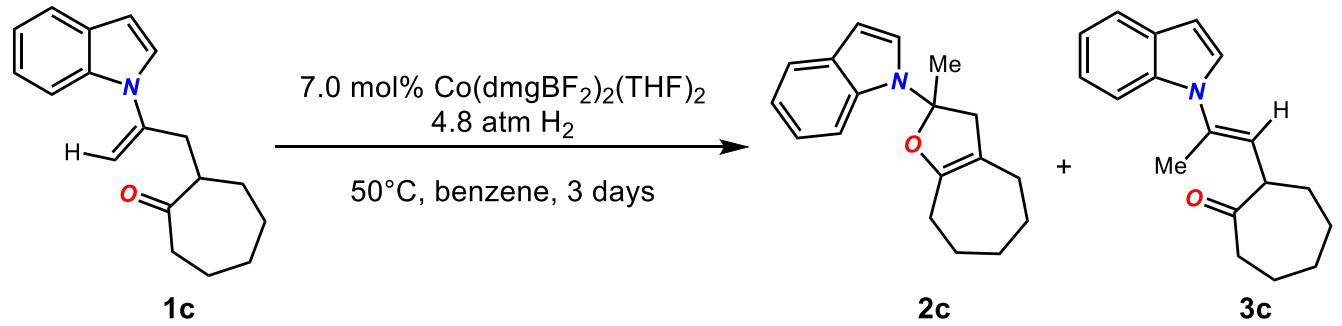

According to general process, 1c $(0.2 \mathrm{mmol})$ was subjected to the reaction condition. The product was purified through chromatography (hexane / EtOAc $=10: 1$ ) afford 2c as a colorless oil. Yield 46\% (24.6 mg). 3c was isolated as a colorless oil. Yield 35\% (18.7 mg).

\section{1-(2-Methyl-3,4,5,6,7,8-hexahydro-2H-cyclohepta[b]furan-2-yl)-1H-indole (2c)}

${ }^{1} \mathrm{H}$ NMR $\left(400 \mathrm{MHz}, \mathrm{CD}_{3} \mathrm{C}(\mathrm{O}) \mathrm{CD}_{3}\right) \delta 7.58-7.54(\mathrm{~m}, 1 \mathrm{H}), 7.50(\mathrm{dq}, J=8.3,0.9 \mathrm{~Hz}, 1 \mathrm{H}), 7.47(\mathrm{~d}$, $J=3.4 \mathrm{~Hz}, 1 \mathrm{H}), 7.12(\mathrm{ddd}, J=8.3,7.0,1.3 \mathrm{~Hz}, 1 \mathrm{H}), 7.04(\mathrm{ddd}, J=8.0,7.0,1.1 \mathrm{~Hz}, 1 \mathrm{H}), 6.43$ $(\mathrm{dd}, J=3.4,0.9 \mathrm{~Hz}, 1 \mathrm{H}), 3.24(\mathrm{~d}, J=15.5 \mathrm{~Hz}, 1 \mathrm{H}), 2.99-2.88(\mathrm{~m}, 1 \mathrm{H}), 2.32(\mathrm{t}, J=3.8 \mathrm{~Hz}, 2 \mathrm{H})$, $2.21-2.08(\mathrm{~m}, 2 \mathrm{H}), 1.91(\mathrm{~s}, 3 \mathrm{H}), 1.72-1.68(\mathrm{~m}, 5 \mathrm{H}), 1.33-1.27(\mathrm{~m}, 1 \mathrm{H}) .{ }^{13} \mathrm{C} \mathrm{NMR}(101 \mathrm{MHz}$, $\left.\mathrm{CD}_{3} \mathrm{C}(\mathrm{O}) \mathrm{CD}_{3}\right) \delta 149.6,134.3,130.3,124.8,121.6,121.2,119.8,112.1,105.5,101.4,96.0,46.0$, 
27.9, 23.3, 23.1, 23.0, 22.9. HRMS (ESI) m/z: $[\mathrm{M}+\mathrm{H}]^{+}$Calcd for $\mathrm{C}_{18} \mathrm{H}_{22} \mathrm{NO} 268.1701$, found 268.1714 .

\section{(E)-2-(2-(1H-indol-1-yl)prop-1-en-1-yl)cycloheptan-1-one (3c)}

${ }^{1} \mathrm{H}$ NMR $\left(400 \mathrm{MHz}, \mathrm{CD}_{3} \mathrm{C}(\mathrm{O}) \mathrm{CD}_{3}\right) \delta 7.57(\mathrm{td}, J=8.0,1.0 \mathrm{~Hz}, 2 \mathrm{H}), 7.32(\mathrm{~d}, J=3.3 \mathrm{~Hz}, 1 \mathrm{H})$, 7.15 (ddd, $J=8.4,7.0,1.3 \mathrm{~Hz}, 1 \mathrm{H}), 7.05(\mathrm{ddd}, J=8.0,7.0,1.1 \mathrm{~Hz}, 1 \mathrm{H}), 6.52(\mathrm{dd}, J=3.3,0.8 \mathrm{~Hz}$, $1 \mathrm{H}), 5.82(\mathrm{dd}, J=9.4,1.2 \mathrm{~Hz}, 1 \mathrm{H}), 3.72(\mathrm{td}, J=9.5,4.0 \mathrm{~Hz}, 1 \mathrm{H}), 2.65-2.49(\mathrm{~m}, 2 \mathrm{H}), 2.21(\mathrm{~d}, J$ $=1.2 \mathrm{~Hz}, 3 \mathrm{H}), 1.93-1.61(\mathrm{~m}, 7 \mathrm{H}), 1.52-1.42(\mathrm{~m}, 1 \mathrm{H}) .{ }^{13} \mathrm{C} \mathrm{NMR}\left(125 \mathrm{MHz}, \mathrm{CD}_{3} \mathrm{C}(\mathrm{O}) \mathrm{CD}_{3}\right) \delta$ 212.4, 136.7, 134.5, 130.0, 127.7, 124.4, 122.6, 121.5, 120.6, 112.1, 103.1, 51.9, 43.4, 33.2, 29.1, 25.1, 17.5. HRMS (ESI) $\mathrm{m} / \mathrm{z}:[\mathrm{M}+\mathrm{H}]^{+}$Calcd for $\mathrm{C}_{18} \mathrm{H}_{22} \mathrm{NO} 268.1701$, found 268.1722.

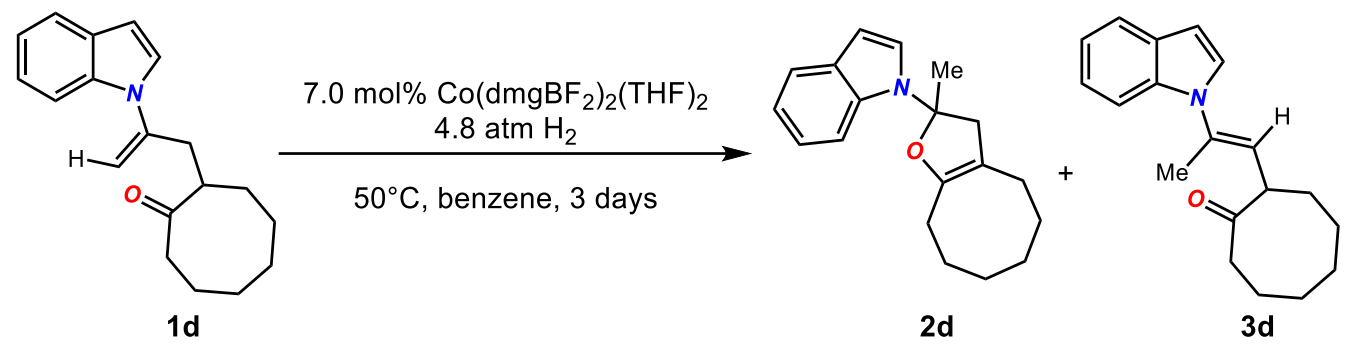

According to general process, $1 \mathrm{~d}(0.2 \mathrm{mmol})$ was subjected to the reaction condition. The product was purified through chromatography (hexane / EtOAc $=10: 1$ ) afford $\mathbf{2 d}$ as a colorless oil. Yield 53\% (29.8 mg). 3d was isolated as a colorless oil. Yield 28\% (15.7 mg).

\section{1-(2-Methyl-3,4,5,6,7,8-hexahydro-2H-cyclohepta[b]furan-2-yl)-1H-indole (2d)}

${ }^{1} \mathrm{H}$ NMR $\left(400 \mathrm{MHz}, \mathrm{CD}_{3} \mathrm{C}(\mathrm{O}) \mathrm{CD}_{3}\right) \delta 7.55(\mathrm{td}, J=7.0,1.1 \mathrm{~Hz}, 2 \mathrm{H}), 7.49(\mathrm{~d}, J=3.4 \mathrm{~Hz}, 1 \mathrm{H})$, 7.12 (ddd, $J=8.2,7.0,1.4 \mathrm{~Hz}, 1 \mathrm{H}), 7.04(\mathrm{ddd}, J=7.9,7.1,1.1 \mathrm{~Hz}, 1 \mathrm{H}), 6.44(\mathrm{dd}, J=3.4,0.8 \mathrm{~Hz}$, $1 \mathrm{H}), 3.25(\mathrm{~d}, J=15.7 \mathrm{~Hz}, 1 \mathrm{H}), 2.94(\mathrm{~d}, J=15.6 \mathrm{~Hz}, 1 \mathrm{H}), 2.34(\mathrm{tt}, J=15.4,7.2 \mathrm{~Hz}, 2 \mathrm{H}), 2.23(\mathrm{q}$, $J=5.4,4.3 \mathrm{~Hz}, 2 \mathrm{H}), 1.91(\mathrm{~s}, 3 \mathrm{H}), 1.78-1.70(\mathrm{~m}, 2 \mathrm{H}), 1.67-1.56(\mathrm{~m}, 6 \mathrm{H}) .{ }^{13} \mathrm{C} \mathrm{NMR}(101 \mathrm{MHz}$, $\left.\mathrm{CD}_{3} \mathrm{C}(\mathrm{O}) \mathrm{CD}_{3}\right) \delta 149.6,134.2,130.2,124.6,121.0,120.7,119.3,112.2,105.8,100.8,95.0,46.7$, 27.2, 26.9, 26.4, 26.0, 25.6, 25.0, 23.7. HRMS (ESI) $\mathrm{m} / \mathrm{z}:[\mathrm{M}+\mathrm{H}]^{+}$Calcd for $\mathrm{C}_{19} \mathrm{H}_{24} \mathrm{NO}$ 282.1858 , found 282.1858 .

\section{(E)-2-(2-(1H-indol-1-yl)prop-1-en-1-yl)cycloheptan-1-one (3d)}

${ }^{1} \mathrm{H}$ NMR $\left(400 \mathrm{MHz}, \mathrm{CD}_{3} \mathrm{C}(\mathrm{O}) \mathrm{CD}_{3}\right) \delta 7.55(\mathrm{td}, J=7.9,1.0 \mathrm{~Hz}, 2 \mathrm{H}), 7.32(\mathrm{~d}, J=3.3 \mathrm{~Hz}, 1 \mathrm{H})$, 7.15 (ddd, $J=8.2,7.0,1.3 \mathrm{~Hz}, 1 \mathrm{H}), 7.05(\mathrm{ddd}, J=8.0,7.0,1.1 \mathrm{~Hz}, 1 \mathrm{H}), 6.52(\mathrm{dd}, J=3.3,0.8 \mathrm{~Hz}$, $1 \mathrm{H}), 5.78(\mathrm{dd}, J=9.4,1.2 \mathrm{~Hz}, 1 \mathrm{H}), 3.72(\mathrm{td}, J=9.8,3.7 \mathrm{~Hz}, 1 \mathrm{H}), 2.59(\mathrm{ddd}, J=13.2,7.7,3.5 \mathrm{~Hz}$, $1 \mathrm{H}$ ), 2.46 (ddd, $J=13.2,10.6,3.5 \mathrm{~Hz}, 1 \mathrm{H}), 2.23(\mathrm{~d}, J=1.2 \mathrm{~Hz}, 3 \mathrm{H}$ ), 2.13 (ddd, $J=14.3,7.2,3.7$ Hz, 1H), $2.02-1.94(\mathrm{~m}, 1 \mathrm{H}), 1.93-1.45(\mathrm{~m}, 7 \mathrm{H}), 1.32$ (dddd, $J=10.0,6.1,4.4,2.2 \mathrm{~Hz}, 1 \mathrm{H})$. 
${ }^{13} \mathrm{C}$ NMR $\left(101 \mathrm{MHz}, \mathrm{CD}_{3} \mathrm{C}(\mathrm{O}) \mathrm{CD}_{3}\right) \delta 215.7,136.6,135.0,130.1,127.7,123.5,122.6,121.6$, 120.6, 112.0, 103.2, 50.9, 41.5, 33.1, 27.7, 27.2, 26.4, 25.4, 17.5. HRMS (ESI) m/z: $[\mathrm{M}+\mathrm{H}]^{+}$ Calcd for $\mathrm{C}_{19} \mathrm{H}_{24} \mathrm{NO} 282.1858$, found 282.1861.
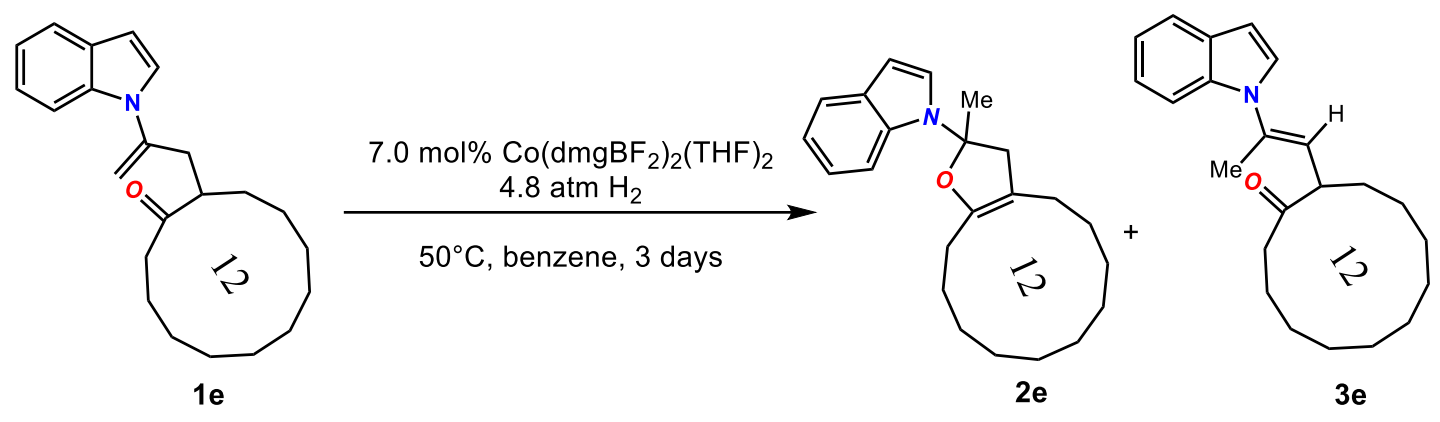

According to general process, 1 e $(0.2 \mathrm{mmol})$ was subjected to the reaction condition. The product was purified through chromatography (hexane / EtOAc $=10: 1$ ) afford $\mathbf{2 e}$ as a colorless oil. Yield 52\% (35.1 mg). 32 was isolated as a colorless oil. Yield 29\% (19.5 mg).

\section{1-(2-Methyl-2,3,4,5,6,7,8,9,10,11,12,13-dodecahydrocyclododeca[b]furan-2-yl)-1H-indole(2e)}

${ }^{1} \mathrm{H}$ NMR $\left(400 \mathrm{MHz}, \mathrm{CD}_{2} \mathrm{Cl}_{2}\right) \delta 7.63-7.56(\mathrm{~m}, 1 \mathrm{H}), 7.45(\mathrm{dd}, J=8.2,1.1 \mathrm{~Hz}, 1 \mathrm{H}), 7.39(\mathrm{~d}, J=$ $3.4 \mathrm{~Hz}, 1 \mathrm{H}), 7.17-7.11(\mathrm{~m}, 1 \mathrm{H}), 7.08(\mathrm{td}, J=7.4,7.0,1.1 \mathrm{~Hz}, 1 \mathrm{H}), 6.46(\mathrm{dd}, J=3.4,0.9 \mathrm{~Hz}$, 1H), $3.20(\mathrm{~d}, J=15.8 \mathrm{~Hz}, 1 \mathrm{H}), 2.76(\mathrm{~d}, J=15.7 \mathrm{~Hz}, 1 \mathrm{H}), 2.35-2.13(\mathrm{~m}, 4 \mathrm{H}), 1.91(\mathrm{~s}, 3 \mathrm{H}), 1.68$ $-1.56(\mathrm{~m}, 2 \mathrm{H}), 1.45-1.27$ (m, 15H). ${ }^{13} \mathrm{C}$ NMR $\left(101 \mathrm{MHz}, \mathrm{CD}_{3} \mathrm{C}(\mathrm{O}) \mathrm{CD}_{3}\right) \delta$ 149.9, 135.4, 131.1, 125.7, 122.0, 121.6, 120.4, 113.2, 108.4, 101.9, 95.6, 45.5, 27.9, 26.0, 25.4, 25.4, 25.1, 25.0, 24.8, 23.3, 22.95, 22.74, 22.56. HRMS (ESI) $\mathrm{m} / \mathrm{z}:[\mathrm{M}+\mathrm{H}]^{+}$Calcd for $\mathrm{C}_{23} \mathrm{H}_{32} \mathrm{NO} 338.2484$, found 338.2485 .

\section{(E)-2-(2-(1H-indol-1-yl)prop-1-en-1-yl)cyclododecan-1-one (3e)}

${ }^{1} \mathrm{H}$ NMR (400 MHz, $\left.\mathrm{CD}_{2} \mathrm{Cl}_{2}\right) \delta 7.59(\mathrm{~d}, J=7.7 \mathrm{~Hz}, 1 \mathrm{H}), 7.47(\mathrm{~d}, J=8.4 \mathrm{~Hz}, 1 \mathrm{H}), 7.17(\mathrm{~d}, J=3.4$ $\mathrm{Hz}, 2 \mathrm{H}), 7.08(\mathrm{t}, J=7.4 \mathrm{~Hz}, 1 \mathrm{H}), 6.54(\mathrm{~d}, J=3.3 \mathrm{~Hz}, 1 \mathrm{H}), 5.72(\mathrm{~d}, J=9.8 \mathrm{~Hz}, 1 \mathrm{H}), 3.64(\mathrm{td}, J=$ 9.9, $3.6 \mathrm{~Hz}, 1 \mathrm{H}), 2.66(\mathrm{td}, J=8.2,7.5,3.5 \mathrm{~Hz}, 1 \mathrm{H}), 2.58-2.52(\mathrm{~m}, 1 \mathrm{H}), 2.25-2.19(\mathrm{~m}, 3 \mathrm{H})$, $1.98(\mathrm{ddd}, J=13.4,9.7,6.1 \mathrm{~Hz}, 2 \mathrm{H}), 1.83(\mathrm{t}, J=12.1 \mathrm{~Hz}, 2 \mathrm{H}), 1.70(\mathrm{~d}, J=9.3 \mathrm{~Hz}, 3 \mathrm{H}), 1.36-$ $1.30(\mathrm{~m}, 11 \mathrm{H}) .{ }^{13} \mathrm{C}$ NMR $\left(101 \mathrm{MHz}, \mathrm{CD}_{2} \mathrm{Cl}_{2}\right) \delta 210.9,134.6,129.0,126.7,122.4,121.9,120.8$, 119.8, 118.2, 111.0, 102.3, 51.1, 37.3, 30.8, 29.7, 25.9, 25.5, 24.1, 23.9, 23.6, 22.3, 22.0, 17.2. HRMS (ESI) m/z: [M+ H $]^{+}$Calcd for $\mathrm{C}_{23} \mathrm{H}_{32} \mathrm{NO} 338.2484$, found 338.2482. 


\section{Cycloisomerization of $\mathrm{N}$-vinyl indoles with $\mathrm{Co}(\mathrm{III}) /$ silane system}

The Co(III)-salen complexes A-I were prepared according to known procedures. ${ }^{4,5}$ Silane was used as a stock solution in Benzene.

\section{General procedure for Cycloisomerization of $N$-vinyl-indoles using $\mathrm{Co}(\mathrm{III}) /$ silane system}

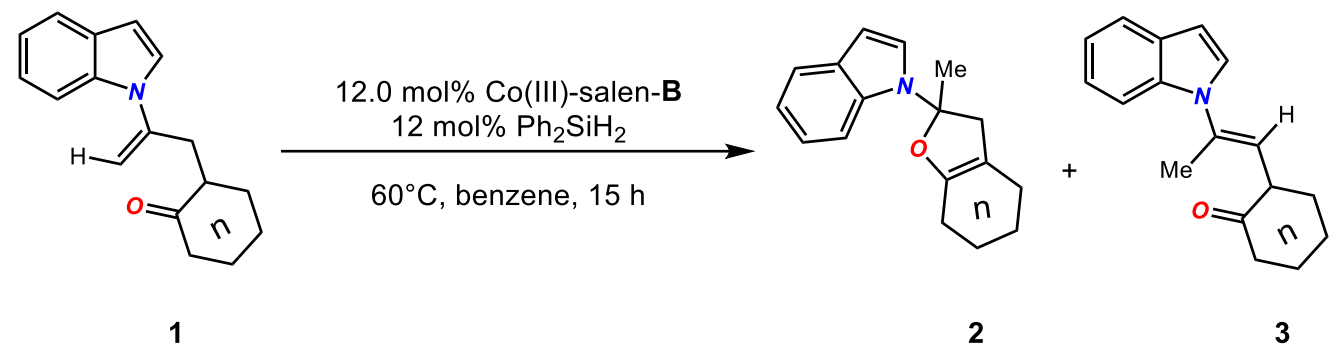

In the glove box, to an oven-dried $6 \mathrm{~mL}$ vial equipped with a stir bar was added the Indole substrates 1 (0.2 mmol) and $\mathrm{Co}(\mathrm{III})$-salen $\mathbf{B}(12 \mathrm{~mol} \%)$. A stock solution of $\mathrm{Ph}_{2} \mathrm{SiH}_{2}$ (2.0 mL, 12 mol\%) was added. The vial was sealed with a white cap and further sealed by black tape. The reaction mixture was removed from glove box, put in pre-heated oil bath $\left(60{ }^{\circ} \mathrm{C}\right)$ for the indicated time. The reaction mixture was cooled to room temperature, filtered through a short plug of silicon gel, and eluted with EtOAc. The resulting filtrate was concentrated and the crude reaction mixture was analysed by ${ }^{1} \mathrm{H}$ NMR $\left(\mathrm{CDCl}_{3}, \mathrm{CD}_{2} \mathrm{Cl}_{2}\right.$ or $\left.\mathrm{CD}_{3} \mathrm{C}(\mathrm{O}) \mathrm{CD}_{3}\right)$ to determine product distribution and selectivity. Ratio reported here is refereed to cycloisomerization (2)/isomerization (3). Unless stated otherwise, the crude product was purified by chromatoaphy on silica gel, concentrated under reduced pressure and stored neat or as a solution in acetone.

\section{$\underline{\text { Representative Procedure for Cycloisomerization reaction on } 1.0 \mathrm{mmol} \text { scale. }}$}

In the glove box, to an oven-dried $25 \mathrm{~mL}$ vial equipped with a stir bar was added the Indole substrates 1 f $(1.0 \mathrm{mmol})$ and $\mathrm{Co}(\mathrm{III})$-salen $\mathbf{B}(12 \mathrm{~mol} \%)$. A stock solution of $\mathrm{Ph}_{2} \mathrm{SiH}_{2}(10.0 \mathrm{~mL}$, $12 \mathrm{~mol} \%$ ) was added. The vial was sealed with a white cap and further sealed by black tape. The reaction mixture was removed from glove box, put in pre-heated oil bath $\left(60^{\circ} \mathrm{C}\right)$ for the indicated time. The reaction mixture was cooled to room temperature, filtered through a short plug of silicon gel, and eluted with EtOAc. The resulting filtrate was concentrated and the crude reaction mixture was analysed by ${ }^{1} \mathrm{H}$ NMR $\left(\mathrm{CD}_{3} \mathrm{C}(\mathrm{O}) \mathrm{CD}_{3}\right)$ to determine product distribution and selectivity. Purification by chromatography on silica gel (hexane / EtOAc $=10: 1$ ) afforded $2 \mathbf{f}$ as a colorless oil. Yield $81 \%(244.1 \mathrm{mg}) .{ }^{1} \mathrm{H}$ NMR $\left(400 \mathrm{MHz}, \mathrm{CD}_{3} \mathrm{C}(\mathrm{O}) \mathrm{CD}_{3}\right) \delta 7.46-7.39(\mathrm{~m}$, 
2H), $7.34(\mathrm{dt}, J=1.7,0.9 \mathrm{~Hz}, 1 \mathrm{H}), 6.95(\mathrm{dd}, J=8.5,1.7 \mathrm{~Hz}, 1 \mathrm{H}), 6.33(\mathrm{dd}, J=3.4,0.9 \mathrm{~Hz}, 1 \mathrm{H})$,

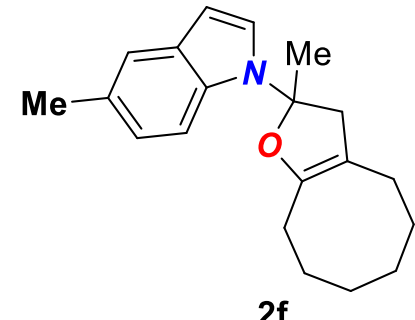

$2 f$ $3.23(\mathrm{~d}, J=15.6 \mathrm{~Hz}, 1 \mathrm{H}), 2.91(\mathrm{~d}, J=15.6 \mathrm{~Hz}, 1 \mathrm{H}), 2.38(\mathrm{~s}, 3 \mathrm{H}), 2.37$ $-2.31(\mathrm{~m}, 2 \mathrm{H}), 2.26-2.19(\mathrm{~m}, 2 \mathrm{H}), 1.89(\mathrm{~s}, 3 \mathrm{H}), 1.77-1.70(\mathrm{~m}, 2 \mathrm{H})$, $1.68-1.56(\mathrm{~m}, 6 \mathrm{H}) .{ }^{13} \mathrm{C} \mathrm{NMR}\left(125 \mathrm{MHz}, \mathrm{CD}_{3} \mathrm{C}(\mathrm{O}) \mathrm{CD}_{3}\right) \delta 150.6$, 133.6, 131.5, 129.1, 125.6, 123.6, 121.3, 112.8, 106.7, 101.3, 95.9, 47.6, 28.2, 27.9, 27.4, 27.0, 26.5, 26.0, 24.6, 21.4. HRMS (ESI) m/z:

$[\mathrm{M}+\mathrm{H}]^{+}$Calcd for $\mathrm{C}_{20} \mathrm{H}_{26} \mathrm{NO} 296.2014$, found 296.2019.

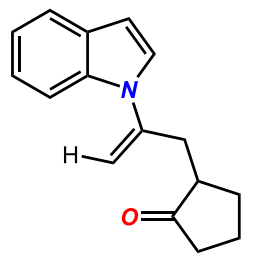

$1 \mathrm{~b}$

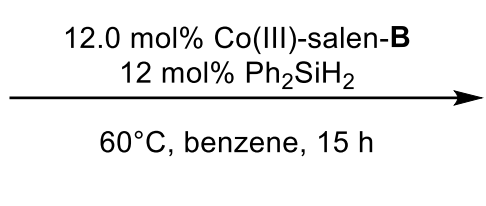

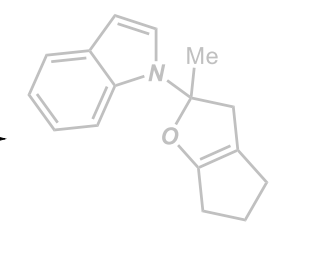

2b

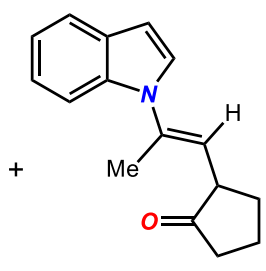

$3 b$

\section{(E)-2-(2-(1H-indol-1-yl)prop-1-en-1-yl)cyclopentan-1-one (3b)}

According to general procedure, $\mathbf{1 b}(0.2 \mathrm{mmol})$ was subjected to the reaction condition. The product was purified through chromatography (hexane / EtOAc $=10: 1$ ) afford $\mathbf{3 b}$ as a colorless oil. Yield 66\% (32.0 mg). ${ }^{1} \mathrm{H}$ NMR (400 MHz, $\left.\mathrm{CD}_{3} \mathrm{C}(\mathrm{O}) \mathrm{CD}_{3}\right) \delta 7.56(\mathrm{~d}, J=9.1 \mathrm{~Hz}, 2 \mathrm{H}), 7.34(\mathrm{~d}$, $J=3.3 \mathrm{~Hz}, 1 \mathrm{H}$ ), 7.15 (ddd, $J=8.1,7.0,1.3 \mathrm{~Hz}, 1 \mathrm{H}), 7.05$ (ddd, $J=8.1,7.0,1.0 \mathrm{~Hz}, 1 \mathrm{H}$ ), 6.53 $(\mathrm{dd}, J=3.3,0.8 \mathrm{~Hz}, 1 \mathrm{H}), 5.49(\mathrm{dd}, J=8.8,1.2 \mathrm{~Hz}, 1 \mathrm{H}), 3.36-3.26(\mathrm{~m}, 1 \mathrm{H}), 2.48-2.39(\mathrm{~m}, 1 \mathrm{H})$, $2.38-2.29$ (m, 1H), 2.23 (d, $J=1.3 \mathrm{~Hz}, 3 \mathrm{H}), 2.22-2.15(\mathrm{~m}, 1 \mathrm{H}), 2.15-2.08(\mathrm{~m}, 1 \mathrm{H}), 2.01-$ $1.91(\mathrm{~m}, 1 \mathrm{H}), 1.91-1.79(\mathrm{~m}, 1 \mathrm{H}) .{ }^{13} \mathrm{C}$ NMR $\left(125 \mathrm{MHz}, \mathrm{CD}_{3} \mathrm{C}(\mathrm{O}) \mathrm{CD}_{3}\right) \delta$ 216.6, 136.1, 136.0, 129.3, 126.9, 121.8, 121.7, 120.8, 119.8, 111.41, 102.4, 48.9, 37.0, 30.7, 20.9, 16.9.

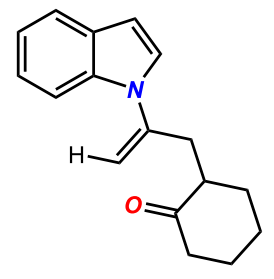

$1 a$

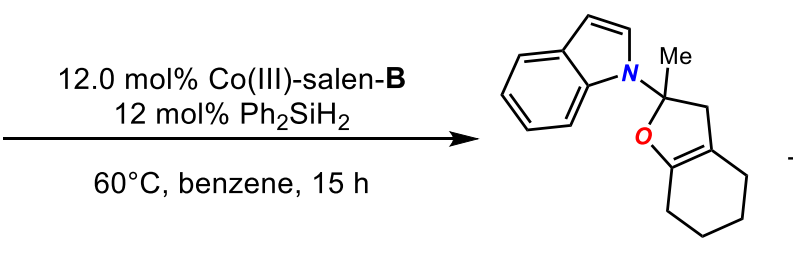

$2 a$

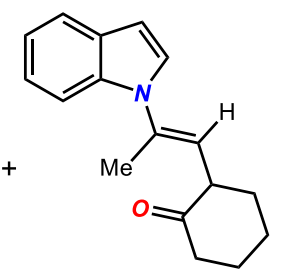

$3 a$

According to general process, 1a $(0.2 \mathrm{mmol})$ was subjected to the reaction condition. The product was purified through chromatography (hexane / EtOAc $=10: 1$ ) afford 2a as a colorless oil. Yield 50\% (25.3 mg). 3a was isolated as a colorless oil. Yield 30\% (15.2 mg).

\section{1-(2-Methyl-2,3,4,5,6,7-hexahydrobenzofuran-2-yl)-1H-indole (2a)}


${ }^{1} \mathrm{H}$ NMR $\left(500 \mathrm{MHz}, \mathrm{CDCl}_{3}\right) \delta 7.63(\mathrm{~d}, J=7.7 \mathrm{~Hz}, 1 \mathrm{H}), 7.43-7.38(\mathrm{~m}, 2 \mathrm{H}), 7.20-7.16(\mathrm{~m}, 1 \mathrm{H})$, $7.15-7.10(\mathrm{~m}, 1 \mathrm{H}), 6.48$ (d, $J=3.4 \mathrm{~Hz}, 1 \mathrm{H}), 3.22$ (d, J = 15.2 Hz, 1H), 2.79 (d, $J=15.4 \mathrm{~Hz}$, $1 \mathrm{H}), 2.18(\mathrm{dt}, J=6.0,3.1 \mathrm{~Hz}, 2 \mathrm{H}), 2.11-2.03(\mathrm{~m}, 2 \mathrm{H}), 1.97(\mathrm{~s}, 3 \mathrm{H}), 1.86-1.77(\mathrm{~m}, 2 \mathrm{H}), 1.77-$ $1.68(\mathrm{~m}, 2 \mathrm{H}) .{ }^{13} \mathrm{C}$ NMR $\left(125 \mathrm{MHz}, \mathrm{CDCl}_{3}\right) \delta 149.6,134.3,130.3,124.8,121.7,121.2,119.8$, 112.1, 105.5, 101.4, 96.0, 46.0, 27.9, 23.3, 23.1, 23.0, 22.9.

\section{(E)-2-(2-(1H-indol-1-yl)prop-1-en-1-yl)cyclopentan-1-one (3a)}

${ }^{1} \mathrm{H}$ NMR (400 MHz, CD $\left.{ }_{3} \mathrm{C}(\mathrm{O}) \mathrm{CD}_{3}\right) \delta 7.70(\mathrm{dd}, J=8.3,0.9 \mathrm{~Hz}, 1 \mathrm{H}), 7.56(\mathrm{~d}, J=7.8 \mathrm{~Hz}, 1 \mathrm{H})$, $7.34(\mathrm{~d}, J=3.3 \mathrm{~Hz}, 1 \mathrm{H}), 7.20-7.12(\mathrm{~m}, 1 \mathrm{H}), 7.05(\mathrm{ddd}, J=7.8,6.9,0.8 \mathrm{~Hz}, 1 \mathrm{H}), 6.53(\mathrm{dd}, J=$ 3.3, $0.9 \mathrm{~Hz}, 1 \mathrm{H}), 5.79(\mathrm{dd}, J=9.2,1.2 \mathrm{~Hz}, 1 \mathrm{H}), 3.64-3.53(\mathrm{~m}, 1 \mathrm{H}), 2.51$ (tdd, $J=12.6,5.8,1.0$ $\mathrm{Hz}, 1 \mathrm{H}), 2.43-2.36(\mathrm{~m}, 1 \mathrm{H}), 2.27-2.20(\mathrm{~m}, 1 \mathrm{H}), 2.16(\mathrm{~d}, J=1.2 \mathrm{~Hz}, 3 \mathrm{H}), 2.13-2.08(\mathrm{~m}, 1 \mathrm{H})$, $1.96-1.87(\mathrm{~m}, 2 \mathrm{H}), 1.79-1.66(\mathrm{~m}, 2 \mathrm{H}) .{ }^{13} \mathrm{C} \mathrm{NMR}\left(101 \mathrm{MHz}, \mathrm{CD}_{3} \mathrm{C}(\mathrm{O}) \mathrm{CD}_{3}\right) \delta 209.5,136.7$, $134.8,130.0,127.5,122.9,122.6,121.5,120.6,112.3,103.1,50.5,42.4,35.6,28.4,25.3,17.3$.

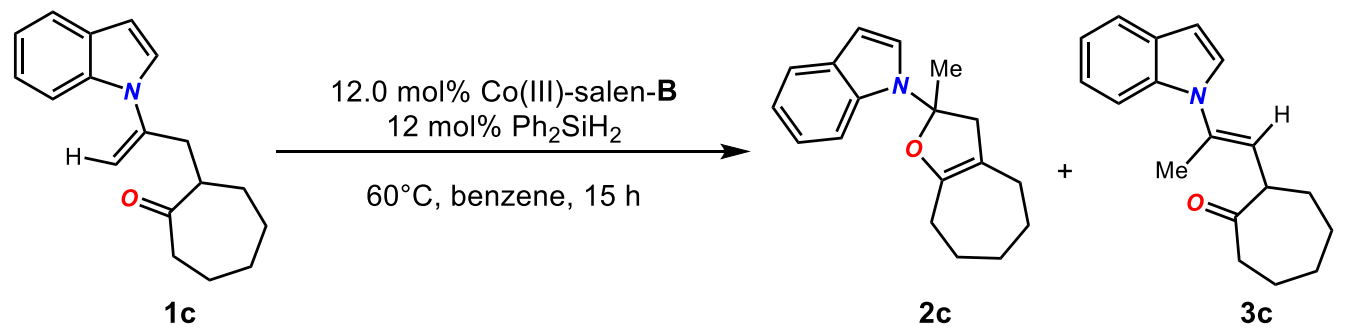

According to general process, 1c $(0.2 \mathrm{mmol})$ was subjected to the reaction condition. The product was purified through chromatography (hexane / EtOAc $=10: 1$ ) afford 2c as a colorless oil. Yield 83\% (44.3 mg). 3c was isolated as a colorless oil. Yield 11\% (5.8 mg).

\section{1-(2-Methyl-3,4,5,6,7,8-hexahydro-2H-cyclohepta[b]furan-2-yl)-1H-indole (2c)}

${ }^{1} \mathrm{H}$ NMR $\left(400 \mathrm{MHz}, \mathrm{CD}_{3} \mathrm{C}(\mathrm{O}) \mathrm{CD}_{3}\right) \delta 7.58-7.54(\mathrm{~m}, 1 \mathrm{H}), 7.50(\mathrm{dq}, J=8.3,0.9 \mathrm{~Hz}, 1 \mathrm{H}), 7.47(\mathrm{~d}$, $J=3.4 \mathrm{~Hz}, 1 \mathrm{H}), 7.12(\mathrm{ddd}, J=8.3,7.0,1.3 \mathrm{~Hz}, 1 \mathrm{H}), 7.04(\mathrm{ddd}, J=8.0,7.0,1.1 \mathrm{~Hz}, 1 \mathrm{H}), 6.43$ $(\mathrm{dd}, J=3.4,0.9 \mathrm{~Hz}, 1 \mathrm{H}), 3.24(\mathrm{~d}, J=15.5 \mathrm{~Hz}, 1 \mathrm{H}), 2.99-2.88(\mathrm{~m}, 1 \mathrm{H}), 2.32(\mathrm{t}, J=3.8 \mathrm{~Hz}, 2 \mathrm{H})$, $2.21-2.08(\mathrm{~m}, 2 \mathrm{H}), 1.91(\mathrm{~s}, 3 \mathrm{H}), 1.72-1.68(\mathrm{~m}, 5 \mathrm{H}), 1.33-1.27(\mathrm{~m}, 1 \mathrm{H}) .{ }^{13} \mathrm{C} \mathrm{NMR}(101 \mathrm{MHz}$, $\left.\mathrm{CD}_{3} \mathrm{C}(\mathrm{O}) \mathrm{CD}_{3}\right) \delta 149.6,134.3,130.3,124.8,121.6,121.2,119.8,112.1,105.5,101.4,96.0,46.0$, 27.9, 23.3, 23.1, 23.0, 22.9.

\section{(E)-2-(2-(1H-indol-1-yl)prop-1-en-1-yl)cycloheptan-1-one (3c)}

${ }^{1} \mathrm{H}$ NMR (400 MHz, $\left.\mathrm{CD}_{3} \mathrm{C}(\mathrm{O}) \mathrm{CD}_{3}\right) \delta 7.57(\mathrm{td}, J=8.0,1.0 \mathrm{~Hz}, 2 \mathrm{H}), 7.32(\mathrm{~d}, J=3.3 \mathrm{~Hz}, 1 \mathrm{H})$, 7.15 (ddd, $J=8.4,7.0,1.3 \mathrm{~Hz}, 1 \mathrm{H}), 7.05(\mathrm{ddd}, J=8.0,7.0,1.1 \mathrm{~Hz}, 1 \mathrm{H}), 6.52(\mathrm{dd}, J=3.3,0.8 \mathrm{~Hz}$, $1 \mathrm{H}), 5.82(\mathrm{dd}, J=9.4,1.2 \mathrm{~Hz}, 1 \mathrm{H}), 3.72(\mathrm{td}, J=9.5,4.0 \mathrm{~Hz}, 1 \mathrm{H}), 2.65-2.49(\mathrm{~m}, 2 \mathrm{H}), 2.21(\mathrm{~d}, J$ 
$=1.2 \mathrm{~Hz}, 3 \mathrm{H}), 1.93-1.61(\mathrm{~m}, 7 \mathrm{H}), 1.52-1.42(\mathrm{~m}, 1 \mathrm{H}) .{ }^{13} \mathrm{C} \mathrm{NMR}\left(125 \mathrm{MHz}, \mathrm{CD}_{3} \mathrm{C}(\mathrm{O}) \mathrm{CD}_{3}\right) \delta$ 212.4, 136.7, 134.5, 130.0, 127.7, 124.4, 122.6, 121.5, 120.6, 112.1, 103.1, 51.9, 43.4, 33.2, 29.1, 25.1, 17.5.

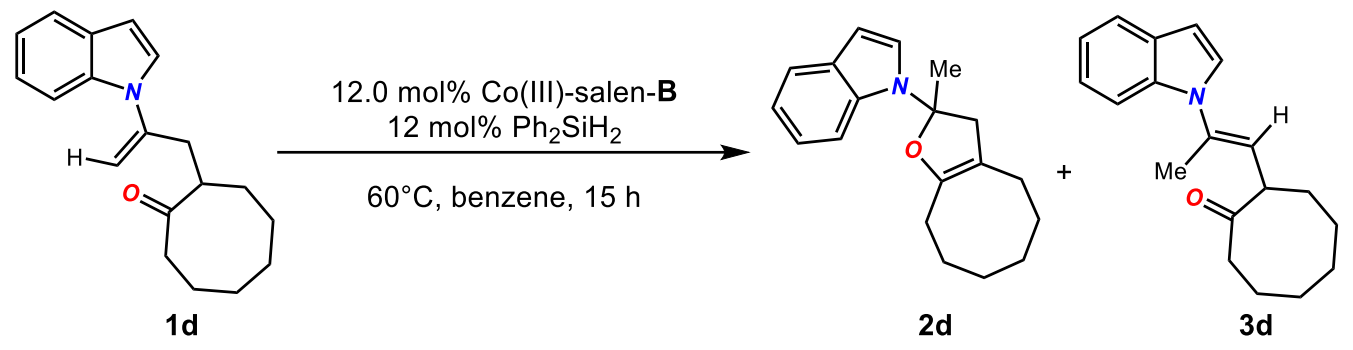

According to general process, $1 \mathbf{d}(0.2 \mathrm{mmol})$ was subjected to the reaction condition. The product was purified through chromatography (hexane / EtOAc $=10: 1$ ) afford $\mathbf{2 d}$ as a colorless oil. Yield $85 \%$ (47.8 mg). 3d was isolated as a colorless oil. Yield 10\% (5.6 mg).

\section{1-(2-Methyl-3,4,5,6,7,8-hexahydro-2H-cyclohepta[b]furan-2-yl)-1H-indole (2d)}

${ }^{1} \mathrm{H}$ NMR $\left(400 \mathrm{MHz}, \mathrm{CD}_{3} \mathrm{C}(\mathrm{O}) \mathrm{CD}_{3}\right) \delta 7.55(\mathrm{td}, J=7.0,1.1 \mathrm{~Hz}, 2 \mathrm{H}), 7.49(\mathrm{~d}, J=3.4 \mathrm{~Hz}, 1 \mathrm{H})$, 7.12 (ddd, $J=8.2,7.0,1.4 \mathrm{~Hz}, 1 \mathrm{H}), 7.04(\mathrm{ddd}, J=7.9,7.1,1.1 \mathrm{~Hz}, 1 \mathrm{H}), 6.44(\mathrm{dd}, J=3.4,0.8 \mathrm{~Hz}$, $1 \mathrm{H}), 3.25(\mathrm{~d}, J=15.7 \mathrm{~Hz}, 1 \mathrm{H}), 2.94(\mathrm{~d}, J=15.6 \mathrm{~Hz}, 1 \mathrm{H}), 2.34(\mathrm{tt}, J=15.4,7.2 \mathrm{~Hz}, 2 \mathrm{H}), 2.23(\mathrm{q}$, $J=5.4,4.3 \mathrm{~Hz}, 2 \mathrm{H}), 1.91(\mathrm{~s}, 3 \mathrm{H}), 1.78-1.70(\mathrm{~m}, 2 \mathrm{H}), 1.67-1.56(\mathrm{~m}, 6 \mathrm{H}) .{ }^{13} \mathrm{C} \mathrm{NMR}(101 \mathrm{MHz}$, $\left.\mathrm{CD}_{3} \mathrm{C}(\mathrm{O}) \mathrm{CD}_{3}\right) \delta 149.6,134.2,130.2,124.6,121.0,120.7,119.3,112.2,105.8,100.8,95.0,46.7$, 27.2, 26.9, 26.4, 26.0, 25.6, 25.0, 23.7.

\section{(E)-2-(2-(1H-indol-1-yl)prop-1-en-1-yl)cycloheptan-1-one (3d)}

${ }^{1} \mathrm{H}$ NMR $\left(400 \mathrm{MHz}, \mathrm{CD}_{3} \mathrm{C}(\mathrm{O}) \mathrm{CD}_{3}\right) \delta 7.55(\mathrm{td}, J=7.9,1.0 \mathrm{~Hz}, 2 \mathrm{H}), 7.32(\mathrm{~d}, J=3.3 \mathrm{~Hz}, 1 \mathrm{H})$, $7.15(\mathrm{ddd}, J=8.2,7.0,1.3 \mathrm{~Hz}, 1 \mathrm{H}), 7.05(\mathrm{ddd}, J=8.0,7.0,1.1 \mathrm{~Hz}, 1 \mathrm{H}), 6.52(\mathrm{dd}, J=3.3,0.8 \mathrm{~Hz}$, $1 \mathrm{H}), 5.78(\mathrm{dd}, J=9.4,1.2 \mathrm{~Hz}, 1 \mathrm{H}), 3.72(\mathrm{td}, J=9.8,3.7 \mathrm{~Hz}, 1 \mathrm{H}), 2.59(\mathrm{ddd}, J=13.2,7.7,3.5 \mathrm{~Hz}$, 1H), 2.46 (ddd, $J=13.2,10.6,3.5 \mathrm{~Hz}, 1 \mathrm{H}), 2.23$ (d, $J=1.2 \mathrm{~Hz}, 3 \mathrm{H}$ ), 2.13 (ddd, $J=14.3,7.2,3.7$ Hz, 1H), $2.02-1.94$ (m, 1H), $1.93-1.45$ (m, 7H), 1.32 (dddd, $J=10.0,6.1,4.4,2.2 \mathrm{~Hz}, 1 \mathrm{H}$ ). ${ }^{13} \mathrm{C}$ NMR (101 MHz, $\left.\mathrm{CD}_{3} \mathrm{C}(\mathrm{O}) \mathrm{CD}_{3}\right) \delta 215.7,136.6,135.0,130.1,127.7,123.5,122.6,121.6$, $120.6,112.0,103.2,50.9,41.5,33.1,27.7,27.2,26.4,25.4,17.5$. 


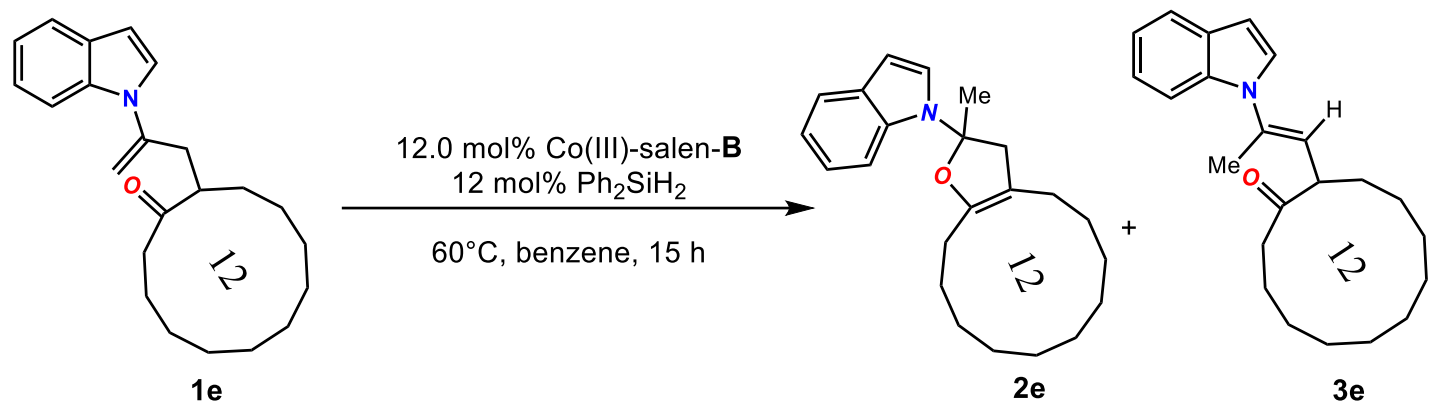

According to general process, 1e $(0.2 \mathrm{mmol})$ was subjected to the reaction condition. The product was purified through chromatography (hexane / EtOAc $=10: 1$ ) afford $2 \mathbf{e}$ as a colorless oil. Yield 72\% (48.5 mg). 3e was isolated as a colorless oil. Yield 22\% (14.8 mg).

\section{1-(2-Methyl-2,3,4,5,6,7,8,9,10,11,12,13-dodecahydrocyclododeca[b]furan-2-yl)-1H-indole(2e)}

${ }^{1} \mathrm{H}$ NMR $\left(400 \mathrm{MHz}, \mathrm{CD}_{2} \mathrm{Cl}_{2}\right) \delta 7.63-7.56(\mathrm{~m}, 1 \mathrm{H}), 7.45(\mathrm{dd}, J=8.2,1.1 \mathrm{~Hz}, 1 \mathrm{H}), 7.39(\mathrm{~d}, J=$ $3.4 \mathrm{~Hz}, 1 \mathrm{H}), 7.17-7.11(\mathrm{~m}, 1 \mathrm{H}), 7.08(\mathrm{td}, J=7.4,7.0,1.1 \mathrm{~Hz}, 1 \mathrm{H}), 6.46(\mathrm{dd}, J=3.4,0.9 \mathrm{~Hz}$, 1H), $3.20(\mathrm{~d}, J=15.8 \mathrm{~Hz}, 1 \mathrm{H}), 2.76(\mathrm{~d}, J=15.7 \mathrm{~Hz}, 1 \mathrm{H}), 2.35-2.13(\mathrm{~m}, 4 \mathrm{H}), 1.91(\mathrm{~s}, 3 \mathrm{H}), 1.68$ - 1.56 (m, 2H), 1.45 - 1.27 (m, 15H). ${ }^{13} \mathrm{C}$ NMR (101 MHz, $\left.\mathrm{CD}_{3} \mathrm{C}(\mathrm{O}) \mathrm{CD}_{3}\right) \delta 149.9,135.4,131.1$, 125.7, 122.0, 121.6, 120.4, 113.2, 108.4, 101.9, 95.6, 45.5, 27.9, 26.0, 25.4, 25.4, 25.1, 25.0, 24.8, 23.3, 23.0, 22.7, 22.6 .

\section{(E)-2-(2-(1H-indol-1-yl)prop-1-en-1-yl)cyclododecan-1-one (3e)}

${ }^{1} \mathrm{H}$ NMR (400 MHz, $\left.\mathrm{CD}_{2} \mathrm{Cl}_{2}\right) \delta 7.59(\mathrm{~d}, J=7.7 \mathrm{~Hz}, 1 \mathrm{H}), 7.47(\mathrm{~d}, J=8.4 \mathrm{~Hz}, 1 \mathrm{H}), 7.17(\mathrm{~d}, J=3.4$ $\mathrm{Hz}, 2 \mathrm{H}), 7.08$ (t, $J=7.4 \mathrm{~Hz}, 1 \mathrm{H}), 6.54(\mathrm{~d}, J=3.3 \mathrm{~Hz}, 1 \mathrm{H}), 5.72(\mathrm{~d}, J=9.8 \mathrm{~Hz}, 1 \mathrm{H}), 3.64(\mathrm{td}, J=$ 9.9, 3.6 Hz, 1H), $2.66(\mathrm{td}, J=8.2,7.5,3.5 \mathrm{~Hz}, 1 \mathrm{H}), 2.58-2.52(\mathrm{~m}, 1 \mathrm{H}), 2.25-2.19(\mathrm{~m}, 3 \mathrm{H})$, $1.98(\mathrm{ddd}, J=13.4,9.7,6.1 \mathrm{~Hz}, 2 \mathrm{H}), 1.83(\mathrm{t}, J=12.1 \mathrm{~Hz}, 2 \mathrm{H}), 1.70$ (d, $J=9.3 \mathrm{~Hz}, 3 \mathrm{H}), 1.36-$ $1.30(\mathrm{~m}, 11 \mathrm{H}) .{ }^{13} \mathrm{C}$ NMR $\left(101 \mathrm{MHz}, \mathrm{CD}_{2} \mathrm{Cl}_{2}\right) \delta 210.9,134.6,129.0,126.7,122.4,121.9,120.8$, $119.8,118.2,111.0,102.3,51.1,37.3,30.8,29.7,25.9,25.5,24.1,23.9,23.6,22.3,22.0,17.2$

2g-2p were prepared according to the general procedure, the ratio of cyclo-isomerization to isomerization was determined by the crude ${ }^{1} \mathrm{H}-\mathrm{NMR}$, the yield refers to the isolated yield of the major product.

Methyl 


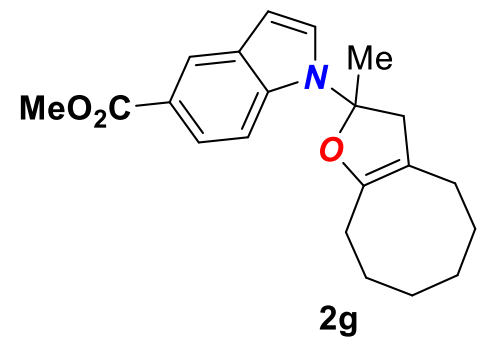

According to general procedure, $\mathbf{1 g}(0.1 \mathrm{mmol})$ was subjected to the reaction condition. Ratio 7.2:1, The product was purified through chromatography (hexane / EtOAc $=10: 1$ to 3:1) afford $\mathbf{2 g}$ as a colorless oil. Yield 56\% (19.0 mg). ${ }^{1} \mathrm{H}$ NMR $(400 \mathrm{MHz}$, $\left.\mathrm{CD}_{3} \mathrm{C}(\mathrm{O}) \mathrm{CD}_{3}\right) \delta 8.31(\mathrm{~d}, J=1.7 \mathrm{~Hz}, 1 \mathrm{H}), 7.81(\mathrm{dd}, J=8.8,1.7 \mathrm{~Hz}$, 1H), $7.66-7.60$ (m, 2H), 6.61 (dd, $J=3.4,0.8 \mathrm{~Hz}, 1 \mathrm{H}), 3.87$ (s, 3H), 3.24 (d, $J=15.8 \mathrm{~Hz}, 1 \mathrm{H})$, $3.01(\mathrm{~d}, J=16.0 \mathrm{~Hz}, 1 \mathrm{H}), 2.37(\mathrm{q}, J=6.2 \mathrm{~Hz}, 2 \mathrm{H}), 2.26-2.20(\mathrm{~m}, 2 \mathrm{H}), 1.94(\mathrm{~s}, 3 \mathrm{H}), 1.74(\mathrm{t}, J=$ $5.9 \mathrm{~Hz}, 2 \mathrm{H}), 1.65-1.56(\mathrm{~m}, 6 \mathrm{H}) .{ }^{13} \mathrm{C}$ NMR $\left(101 \mathrm{MHz}, \mathrm{CD}_{3} \mathrm{C}(\mathrm{O}) \mathrm{CD}_{3}\right) \delta 168.1,150.6,137.6$, 130.7, 127.4, 124.3, 123.1, 122.6, 112.9, 107.1, 103.3, 96.3, 51.9, 47.9, 28.1, 27.9, 27.4, 26.9, 26.5, 25.9, 24.6. HRMS (ESI) m/z: $[\mathrm{M}+\mathrm{H}]^{+}$Calcd for $\mathrm{C}_{21} \mathrm{H}_{26} \mathrm{NO}_{3} 340.1913$, found 340.1908 .

\section{4-Methoxy-1-(2-methyl-2,3,4,5,6,7,8,9-octahydrocycloocta[b]furan-2-yl)-1H-indole (2h)}

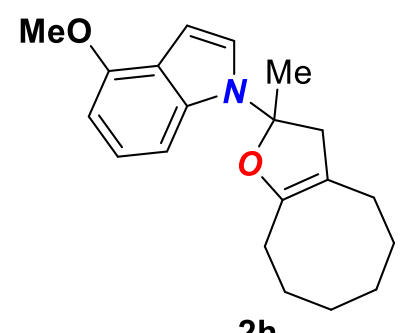

According to general procedure, $\mathbf{1 h}(0.1 \mathrm{mmol})$ was subjected to the reaction condition. Ratio 7.5:1, The product was purified through chromatography (hexane / EtOAc $=6: 1$ ) afford $2 \mathbf{h}$ as a colorless oil. Yield 75\% (23.3 mg). ${ }^{1} \mathrm{H}$ NMR (400 MHz, $\left.\mathrm{CD}_{3} \mathrm{C}(\mathrm{O}) \mathrm{CD}_{3}\right) \delta 7.38$ (d, $J$ $=3.4 \mathrm{~Hz}, 1 \mathrm{H}), 7.14(\mathrm{dd}, J=8.4,0.7 \mathrm{~Hz}, 1 \mathrm{H}), 7.04(\mathrm{t}, J=8.0 \mathrm{~Hz}, 1 \mathrm{H})$, $6.55(\mathrm{~d}, J=7.7 \mathrm{~Hz}, 1 \mathrm{H}), 6.48(\mathrm{~d}, J=3.4 \mathrm{~Hz}, 1 \mathrm{H}), 3.90(\mathrm{~s}, 3 \mathrm{H}), 3.23(\mathrm{~d}$, $J=15.6 \mathrm{~Hz}, 1 \mathrm{H}), 2.93(\mathrm{~d}, J=15.6 \mathrm{~Hz}, 1 \mathrm{H}), 2.40-2.27(\mathrm{~m}, 2 \mathrm{H}), 2.22(\mathrm{td}, J=6.5,4.1 \mathrm{~Hz}, 2 \mathrm{H})$, $1.90(\mathrm{~s}, 3 \mathrm{H}), 1.77-1.70(\mathrm{~m}, 2 \mathrm{H}), 1.65-1.53(\mathrm{~m}, 6 \mathrm{H}) \cdot{ }^{13} \mathrm{C} \mathrm{NMR}\left(101 \mathrm{MHz}, \mathrm{CD}_{3} \mathrm{C}(\mathrm{O}) \mathrm{CD}_{3}\right) \delta$ 153.46, 149.65, 135.54, 122.97, 122.04, 120.69, 105.90, 105.63, 99.38, 98.06, 95.10, 54.50, 46.80, 27.26, 26.99, 26.52, 26.04, 25.62, 25.03, 23.73. HRMS (ESI) m/z: $[\mathrm{M}+\mathrm{H}]^{+}$Calcd for $\mathrm{C}_{20} \mathrm{H}_{26} \mathrm{NO}_{2} 312.1964$, found 312.1962 .

Methyl 1-(2-methyl-2,3,4,5,6,7,8,9-octahydrocycloocta[b]furan-2-yl)-1H-indole-4carboxylate (2i)

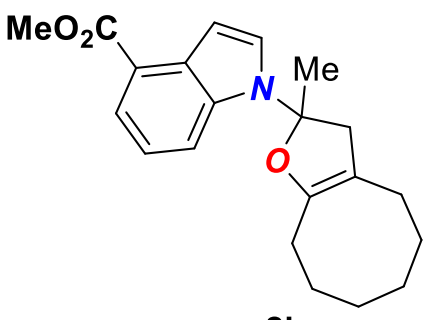

2i

According to general procedure, $\mathbf{1 i}(0.1 \mathrm{mmol})$ was subjected to the reaction condition. Ratio 6.7:1, The product was purified through chromatography (hexane / EtOAc $=10: 1$ to 3:1) afford $\mathbf{2} \mathbf{i}$ as a colorless oil. Yield $61 \% \quad(20.7 \quad \mathrm{mg}) .{ }^{1} \mathrm{H}$ NMR $(400 \mathrm{MHz}$, $\left.\mathrm{CD}_{3} \mathrm{C}(\mathrm{O}) \mathrm{CD}_{3}\right) \delta 7.84(\mathrm{~d}, J=7.8 \mathrm{~Hz}, 2 \mathrm{H}), 7.68(\mathrm{~d}, J=3.4 \mathrm{~Hz}, 1 \mathrm{H})$, 
$7.23(\mathrm{t}, J=7.9 \mathrm{~Hz}, 1 \mathrm{H}), 7.09(\mathrm{~d}, J=3.3 \mathrm{~Hz}, 1 \mathrm{H}), 3.92(\mathrm{~s}, 3 \mathrm{H}), 3.24(\mathrm{~d}, J=15.8 \mathrm{~Hz}, 1 \mathrm{H}), 3.00(\mathrm{~d}$, $J=15.8 \mathrm{~Hz}, 1 \mathrm{H}), 2.37(\mathrm{dt}, J=14.4,7.7 \mathrm{~Hz}, 2 \mathrm{H}), 2.24(\mathrm{q}, J=5.6,4.7 \mathrm{~Hz}, 2 \mathrm{H}), 1.95(\mathrm{~s}, 3 \mathrm{H}), 1.73$ $(\mathrm{q}, J=6.0 \mathrm{~Hz}, 2 \mathrm{H}), 1.66-1.56(\mathrm{~m}, 6 \mathrm{H}) .{ }^{13} \mathrm{C} \mathrm{NMR}\left(101 \mathrm{MHz}, \mathrm{CD}_{3} \mathrm{C}(\mathrm{O}) \mathrm{CD}_{3}\right) \delta 168.0,150.6$, 136.0, 130.6, 127.9, 123.6, 122.5, 121.2, 118.0, 107.0, 103.0, 96.2, 51.8, 47.9, 28.1, 28.0, 27.4, 26.9, 26.5, 25.9, 24.6. HRMS (ESI) $\mathrm{m} / \mathrm{z}$ : $[\mathrm{M}+\mathrm{H}]^{+}$Calcd for $\mathrm{C}_{21} \mathrm{H}_{26} \mathrm{NO}_{3}$ 340.1913, found 340.1915 .

\section{1-(2-Methyl-2,3,4,5,6,7,8,9-octahydrocycloocta[b]furan-2-yl)-1H-indole-4-carbonitrile (2j)}

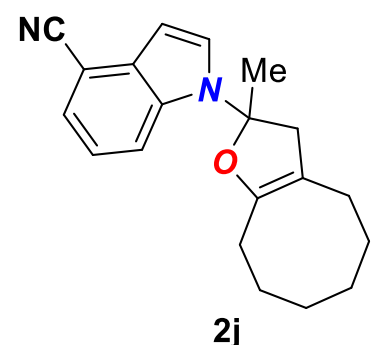

2j
According to general procedure, $\mathbf{1 j}(0.1 \mathrm{mmol})$ was subjected to the reaction condition. Ratio 6.1:1, The product was purified through chromatography (hexane / EtOAc $=7: 1$ ) afford $\mathbf{2} \mathbf{j}$ as a colorless oil. Yield 52\% (15.9 mg). ${ }^{1} \mathrm{H}$ NMR (400 MHz, $\left.\mathrm{CD}_{3} \mathrm{C}(\mathrm{O}) \mathrm{CD}_{3}\right) \delta 7.92(\mathrm{~d}, J=$ $8.4 \mathrm{~Hz}, 1 \mathrm{H}), 7.79(\mathrm{~d}, J=3.4 \mathrm{~Hz}, 1 \mathrm{H}), 7.52(\mathrm{dd}, J=7.4,0.8 \mathrm{~Hz}, 1 \mathrm{H}), 7.29$ $(\mathrm{dd}, J=8.5,7.4 \mathrm{~Hz}, 1 \mathrm{H}), 6.64(\mathrm{dd}, J=3.4,0.9 \mathrm{~Hz}, 1 \mathrm{H}), 3.24(\mathrm{~d}, J=15.9$

$\mathrm{Hz}, 1 \mathrm{H}), 3.04(\mathrm{~d}, J=16.0 \mathrm{~Hz}, 1 \mathrm{H}), 2.42-2.31(\mathrm{~m}, 2 \mathrm{H}), 2.23$ (q, $J=6.5,5.9 \mathrm{~Hz}, 2 \mathrm{H}), 1.96$ (s, $3 \mathrm{H}), 1.73(\mathrm{p}, J=5.9 \mathrm{~Hz}, 2 \mathrm{H}), 1.67-1.53(\mathrm{~m}, 6 \mathrm{H}) .{ }^{13} \mathrm{C} \mathrm{NMR}\left(101 \mathrm{MHz}, \mathrm{CD}_{3} \mathrm{C}(\mathrm{O}) \mathrm{CD}_{3}\right) \delta 150.6$, 134.9, 132.0, 129.0, 125.6, 122.0, 118.9, 118.3, 107.3, 103.9, 100.2, 96.5, 48.0, 28.1, 28.0, 27.4, 26.9, 26.5, 25.9, 24.6. HRMS (ESI) m/z: $[\mathrm{M}+\mathrm{H}]^{+}$Calcd for $\mathrm{C}_{20} \mathrm{H}_{23} \mathrm{~N}_{2} \mathrm{O}$ 307.1810, found 307.1816 .

6-Chloro-1-(2-methyl-2,3,4,5,6,7,8,9-octahydrocycloocta[b]furan-2-yl)-1H-indole (2k)

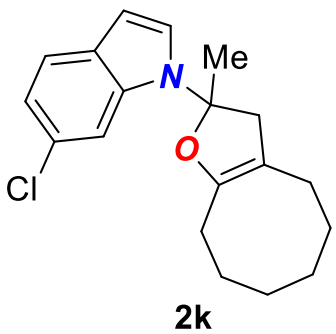

According to general procedure, $1 \mathbf{k}(0.1 \mathrm{mmol})$ was subjected to the reaction condition. Ratio $>20: 1$, The product was purified through chromatography (hexane / EtOAc $=10: 1$ ) afford $2 \mathbf{k}$ as a colorless oil. Yield $65 \%(15.8 \mathrm{mg})$. When the reaction was run at $80^{\circ} \mathrm{C}$, ratio $11: 1$. Yield $80 \%$ determined by ${ }^{1} \mathrm{H}-\mathrm{NMR} .{ }^{1} \mathrm{H}$ NMR $\left(400 \mathrm{MHz}, \mathrm{CD}_{3} \mathrm{C}(\mathrm{O}) \mathrm{CD}_{3}\right) \delta 7.60$ $(\mathrm{dd}, J=1.8,0.9 \mathrm{~Hz}, 1 \mathrm{H}), 7.56(\mathrm{~d}, J=8.4 \mathrm{~Hz}, 1 \mathrm{H}), 7.53$ (d, $J=3.4 \mathrm{~Hz}, 1 \mathrm{H}), 7.06$ (dd, $J=8.4,1.9$ Hz, 1H), 6.47 (dd, $J=3.4,0.9 \mathrm{~Hz}, 1 \mathrm{H}), 3.21$ (d, $J=15.8 \mathrm{~Hz}, 1 \mathrm{H}), 2.95$ (d, $J=15.8 \mathrm{~Hz}, 1 \mathrm{H}), 2.36$ $(\mathrm{tt}, J=6.1,1.6 \mathrm{~Hz}, 2 \mathrm{H}), 2.29-2.22(\mathrm{~m}, 2 \mathrm{H}), 1.93(\mathrm{~s}, 3 \mathrm{H}), 1.75(\mathrm{~d}, J=6.9 \mathrm{~Hz}, 2 \mathrm{H}), 1.68-1.58$ $(\mathrm{m}, 6 \mathrm{H}) .{ }^{13} \mathrm{C} \mathrm{NMR}\left(101 \mathrm{MHz}, \mathrm{CD}_{3} \mathrm{C}(\mathrm{O}) \mathrm{CD}_{3}\right) \delta 150.6,135.6,129.8,127.6,126.8,122.8,120.8$, 
113.1, 107.0, 102.2, 96.1, 47.8, 28.0, 27.8, 27.29, 27.0, 26.6, 26.0, 24.7. HRMS (ESI) m/z: [M + $\mathrm{H}]^{+}$Calcd for $\mathrm{C}_{19} \mathrm{H}_{23} \mathrm{ClNO} 316.1468$, found 316.1467 .

\section{6-Fluoro-1-(2-methyl-2,3,4,5,6,7,8,9-octahydrocycloocta[b]furan-2-yl)-1H-indole (2l)}

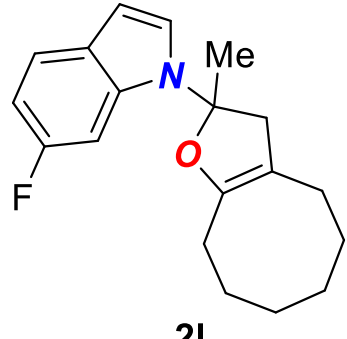

2I

According to general procedure, 11 ( $0.1 \mathrm{mmol})$ was subjected to the reaction condition. Ratio 10:1, The product was purified through chromatography (hexane / EtOAc $=10: 1$ ) afford $2 \mathbf{l}$ as a colorless oil. Yield 71\% (15.9 mg). ${ }^{1} \mathrm{H}$ NMR (400 MHz, $\left.\mathrm{CD}_{3} \mathrm{C}(\mathrm{O}) \mathrm{CD}_{3}\right) \delta 7.55$ (dd, $J=$ 8.6, $5.6 \mathrm{~Hz}, 1 \mathrm{H}), 7.49(\mathrm{~d}, J=3.5 \mathrm{~Hz}, 1 \mathrm{H}), 7.28(\mathrm{dd}, J=10.7,1.7 \mathrm{~Hz}, 1 \mathrm{H})$, $6.94-6.81(\mathrm{~m}, 1 \mathrm{H}), 6.46(\mathrm{~d}, J=3.5 \mathrm{~Hz}, 1 \mathrm{H}), 3.21(\mathrm{~d}, J=15.8 \mathrm{~Hz}, 1 \mathrm{H})$, $2.94(\mathrm{~d}, J=15.8 \mathrm{~Hz}, 1 \mathrm{H}), 2.37$ (ddd, $J=20.8,14.5,6.4 \mathrm{~Hz}, 2 \mathrm{H}), 2.29-2.19$ (m, 2H), 1.91 (s, $3 \mathrm{H}), 1.73(\mathrm{q}, J=6.5,6.0 \mathrm{~Hz}, 2 \mathrm{H}), 1.70-1.55(\mathrm{~m}, 6 \mathrm{H}) .{ }^{13} \mathrm{C} \mathrm{NMR}\left(125 \mathrm{MHz}, \mathrm{CD}_{3} \mathrm{C}(\mathrm{O}) \mathrm{CD}_{3}\right) \delta$ $159.1\left(\mathrm{~d}, J^{\mathrm{F}}=245.0 \mathrm{~Hz}\right), 149.7,134.2\left(\mathrm{~d}, J^{\mathrm{F}}=12.5 \mathrm{~Hz}\right), 126.8,125.4\left(\mathrm{~d}, J^{\mathrm{F}}=3.8 \mathrm{~Hz}\right), 121.6\left(\mathrm{~d}, J^{\mathrm{F}}\right.$ $=11.2 \mathrm{~Hz}), 107.8\left(\mathrm{~d}, J^{\mathrm{F}}=25.0 \mathrm{~Hz}\right), 106.0,101.2,98.6\left(\mathrm{~d}, J^{\mathrm{F}}=27.5 \mathrm{~Hz}\right), 95.2,46.5,27.2,26.9$, 26.5, 26.0, 25.6, 25.0, 23.7. ${ }^{19} \mathrm{~F}$ NMR (376 MHz, $\left.\mathrm{CD}_{3} \mathrm{C}(\mathrm{O}) \mathrm{CD}_{3}\right) \delta$-122.70. HRMS (ESI-) m/z: $[\mathrm{M}+\mathrm{H}]^{+}$Calcd for $\mathrm{C}_{19} \mathrm{H}_{22} \mathrm{FNO} 300.1764$, found 300.1778 .

\section{5-(4-Methoxyphenyl)-1-(2-methyl-2,3,4,5,6,7,8,9-octahydrocycloocta[b]furan-2-yl)-1H-} indole $(2 \mathrm{~m})$

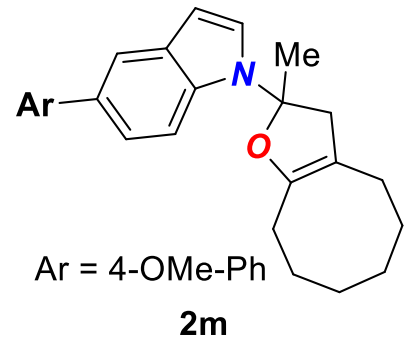

According to general procedure, $11(0.1 \mathrm{mmol})$ was subjected to the reaction condition. Ratio 6.8:1, The product was purified through chromatography (hexane / EtOAc $=4: 1$ ) afford $\mathbf{2} \mathbf{m}$ as a colorless oil. Yield 68\% (26.3 mg). ${ }^{1} \mathrm{H} \mathrm{NMR}\left(400 \mathrm{MHz}, \mathrm{CD}_{3} \mathrm{C}(\mathrm{O}) \mathrm{CD}_{3}\right) \delta 7.77(\mathrm{~d}, J$ $=1.8 \mathrm{~Hz}, 1 \mathrm{H}), 7.64-7.57(\mathrm{~m}, 3 \mathrm{H}), 7.51(\mathrm{~d}, J=3.4 \mathrm{~Hz}, 1 \mathrm{H}), 7.38(\mathrm{dd}$, $J=8.6,1.8 \mathrm{~Hz}, 1 \mathrm{H}), 7.00(\mathrm{~d}, J=8.8 \mathrm{~Hz}, 2 \mathrm{H}), 6.48(\mathrm{~d}, J=4.0 \mathrm{~Hz}, 1 \mathrm{H})$,

$3.84(\mathrm{~s}, 3 \mathrm{H}), 3.28(\mathrm{~d}, J=15.7 \mathrm{~Hz}, 1 \mathrm{H}), 3.04-2.91(\mathrm{~m}, 1 \mathrm{H}), 2.37$ (ddd, $J=8.3,7.0,5.3 \mathrm{~Hz}, 2 \mathrm{H})$, $2.25(\mathrm{q}, J=5.4,4.5 \mathrm{~Hz}, 2 \mathrm{H}), 1.94(\mathrm{~s}, 3 \mathrm{H}), 1.79-1.72(\mathrm{~m}, 2 \mathrm{H}), 1.67-1.55(\mathrm{~m}, 6 \mathrm{H}) .{ }^{13} \mathrm{C}$ NMR $\left(125 \mathrm{MHz}, \mathrm{CD}_{3} \mathrm{C}(\mathrm{O}) \mathrm{CD}_{3}\right) \delta 159.6,150.6,135.6,134.4,133.4,131.8,128.8,126.3,121.4,119.4$, 115.0, 113.4, 106.8, 102.2, 96.0, 55.6, 47.7, 28.2, 27.9, 27.4, 27.0, 26.6, 26.0, 24.6. HRMS (ESI) $\mathrm{m} / \mathrm{z}:[\mathrm{M}+\mathrm{H}]^{+}$Calcd for $\mathrm{C}_{26} \mathrm{H}_{30} \mathrm{NO}_{2} 388.2277$, found 388.2280. 
4-Methoxy-1-(2-methyl-3,4,5,6,7,8-hexahydro-2H-cyclohepta[b]furan-2-yl)-1H-indole (2n)

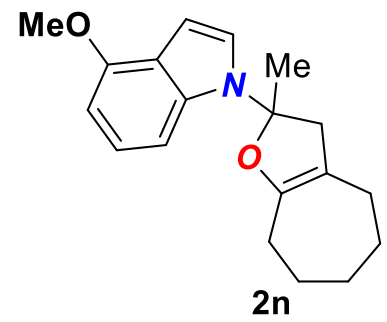

According to general procedure, $1 \mathrm{n}(0.1 \mathrm{mmol})$ was subjected to the reaction condition. Ratio 6.5:1, The product was purified through chromatography (hexane / EtOAc $=7: 1$ ) afford 2n as a colorless oil. Yield $69 \%$ (20.5 mg). ${ }^{1} \mathrm{H}$ NMR (400 MHz, $\left.\mathrm{CD}_{3} \mathrm{C}(\mathrm{O}) \mathrm{CD}_{3}\right) \delta 7.37$ (d, $J=$ $3.4 \mathrm{~Hz}, 1 \mathrm{H}), 7.14-7.02(\mathrm{~m}, 2 \mathrm{H}), 6.57$ (dd, $J=7.5,0.9 \mathrm{~Hz}, 1 \mathrm{H}), 6.49$ $(\mathrm{dd}, J=3.4,0.8 \mathrm{~Hz}, 1 \mathrm{H}), 3.92(\mathrm{~s}, 3 \mathrm{H}), 3.23(\mathrm{~d}, J=15.4 \mathrm{~Hz}, 1 \mathrm{H}), 2.94(\mathrm{~d}, J=15.7 \mathrm{~Hz}, 1 \mathrm{H}), 2.33$ $(\mathrm{s}, 2 \mathrm{H}), 2.13(\mathrm{~d}, J=5.3 \mathrm{~Hz}, 2 \mathrm{H}), 1.90(\mathrm{~s}, 3 \mathrm{H}), 1.80-1.64(\mathrm{~m}, 6 \mathrm{H}) .{ }^{13} \mathrm{C} \mathrm{NMR}(101 \mathrm{MHz}$, $\left.\mathrm{CD}_{3} \mathrm{C}(\mathrm{O}) \mathrm{CD}_{3}\right) \delta 153.5,150.6,135.6,134.1,130.7,127.9,123.0,122.2,108.0,105.6,99.4,98.1$, 94.9, 54.5, 48.6, 27.7, 26.9, 26.0, 25.8. HRMS (ESI) m/z: $[\mathrm{M}+\mathrm{H}]^{+}$Calcd for $\mathrm{C}_{19} \mathrm{H}_{24} \mathrm{NO}_{2}$ 298.1807, found 298.1805 .

Methyl 1-(2-methyl-3,4,5,6,7,8-hexahydro-2H-cyclohepta[b]furan-2-yl)-1H-indole-5carboxylate (2o)

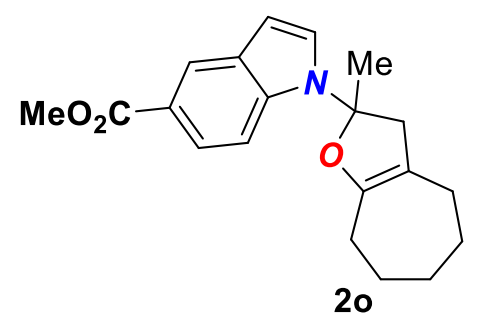

According to general procedure, $10(0.1 \mathrm{mmol})$ was subjected to the reaction condition. The product was purified through chromatography (hexane / EtOAc $=10: 1$ ) afford 20 as a colorless oil. Yield 58\% (18.9 mg). ${ }^{1} \mathrm{H}$ NMR (400 MHz, $\left.\mathrm{CD}_{3} \mathrm{C}(\mathrm{O}) \mathrm{CD}_{3}\right) \delta$ $8.32(\mathrm{dd}, J=1.7,0.7 \mathrm{~Hz}, 1 \mathrm{H}), 7.84(\mathrm{dd}, J=8.8,1.7 \mathrm{~Hz}, 1 \mathrm{H}), 7.67-$ $7.57(\mathrm{~m}, 2 \mathrm{H}), 6.62(\mathrm{dd}, J=3.5,0.8 \mathrm{~Hz}, 1 \mathrm{H}), 3.89(\mathrm{~s}, 3 \mathrm{H}), 3.24(\mathrm{~d}, J=15.9 \mathrm{~Hz}, 1 \mathrm{H}), 3.02(\mathrm{~d}, J=$ $17.9 \mathrm{~Hz}, 1 \mathrm{H}), 2.41-2.31(\mathrm{~m}, 2 \mathrm{H}), 2.19-2.10(\mathrm{~m}, 2 \mathrm{H}), 1.95(\mathrm{~s}, 3 \mathrm{H}), 1.72$ (q, J = 2.6 Hz, 6H). ${ }^{13} \mathrm{C}$ NMR (101 MHz, $\left.\mathrm{CD}_{3} \mathrm{C}(\mathrm{O}) \mathrm{CD}_{3}\right) \delta 168.1,151.6,137.6,130.7,127.4,124.2,123.2,122.6$, 112.9, 109.1, 103.2, 96.1, 51.9, 49.7, 28.6, 27.8, 26.9, 26.6. HRMS (ESI) m/z: $[\mathrm{M}+\mathrm{H}]^{+}$Calcd for $\mathrm{C}_{20} \mathrm{H}_{24} \mathrm{NO}_{3} 326.1756$, found 326.1764 .

\section{6-Chloro-1-(2-methyl-3,4,5,6,7,8-hexahydro-2H-cyclohepta[b]furan-2-yl)-1H-indole (2p)}

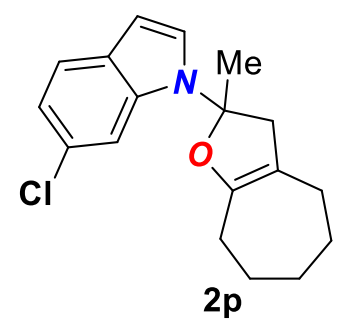

According to general procedure, 1p $(0.1 \mathrm{mmol})$ was subjected to the reaction condition. Ratio 13:1, The product was purified through chromatography (hexane / EtOAc $=10: 1$ ) afford $\mathbf{2 p}$ as a colorless oil. Yield $68 \%(20.5 \mathrm{mg}) .{ }^{1} \mathrm{H} \mathrm{NMR}\left(400 \mathrm{MHz}, \mathrm{CD}_{3} \mathrm{C}(\mathrm{O}) \mathrm{CD}_{3}\right) \delta 7.62-7.54(\mathrm{~m}, 2 \mathrm{H})$, $7.52(\mathrm{~d}, J=3.4 \mathrm{~Hz}, 1 \mathrm{H}), 7.08(\mathrm{dd}, J=8.5,1.8 \mathrm{~Hz}, 1 \mathrm{H}), 6.48(\mathrm{dd}, J=3.4$, 
$0.9 \mathrm{~Hz}, 1 \mathrm{H}), 3.19$ (d, $J=15.9 \mathrm{~Hz}, 1 \mathrm{H}), 2.98(\mathrm{~d}, J=16.0 \mathrm{~Hz}, 1 \mathrm{H}), 2.39-2.27$ (m, 2H), 2.16 (t, $J$ $=7.1 \mathrm{~Hz}, 2 \mathrm{H}), 1.94(\mathrm{~s}, 3 \mathrm{H}), 1.82-1.65(\mathrm{~m}, 6 \mathrm{H}) .{ }^{13} \mathrm{C} \mathrm{NMR}\left(101 \mathrm{MHz}, \mathrm{CD}_{3} \mathrm{C}(\mathrm{O}) \mathrm{CD}_{3}\right) \delta 150.8$, $128.8,127.9,126.8,125.9,121.8,119.8,112.2$, 108.1, 101.2, 95.0, 48.6, 27.7, 26.9, 26.0, 25.7. HRMS (ESI) m/z: [M + H] $]^{+}$Calcd for $\mathrm{C}_{18} \mathrm{H}_{21} \mathrm{ClNO} 301.1312$, found 301.1314. 


\section{Mechanistic Studies}

A. Incorporation of deuterium.

Deuterium incorporation experiments were run according to the general procedure under $\mathrm{H}_{2}$ except under $\mathrm{D}_{2}$. The $\mathrm{D}$ incorporation was determined from crude ${ }^{1} \mathrm{H}-\mathrm{NMR}$ of the reaction mixture (measuring the amount of ${ }^{1} \mathrm{H}$ signal was missing) and further verified by ${ }^{2} \mathrm{H}-\mathrm{NMR}$.

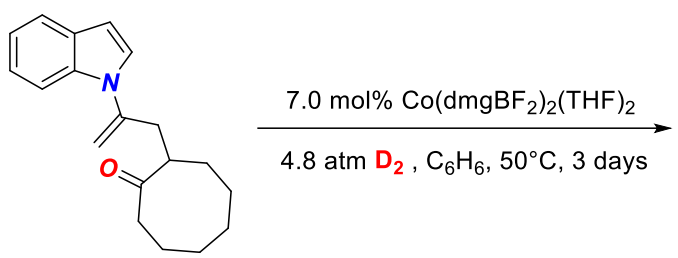

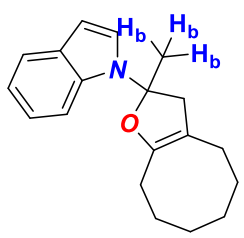

$H_{b}: 30 \% D$ incorporation

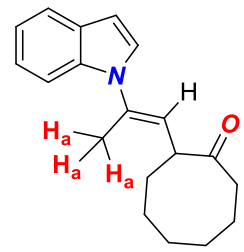

$H_{a}: 21 \%$ D incorporation

B. Reaction time and the ratio of product:

Inside glovebox, indole substrates $1(0.1 \mathrm{mmol})$ and Co(III)-salen $\mathbf{B}(12 \mathrm{~mol} \%)$ were weight out, followed by addition of $\mathrm{Ph}_{2} \mathrm{SiH}_{2}$ in stock solution in $\mathrm{C}_{6} \mathrm{D}_{6}(1.0 \mathrm{~mL}, 12 \mathrm{~mol} \%)$, then the reaction mixture was transfer into a $J$-Young tube, and the $J$-Young tube was placed under pre-heated oil bath $\left(60^{\circ} \mathrm{C}\right)$, crude ${ }^{1} \mathrm{H}-\mathrm{NMR}$ was taken for indicated time, the results are showing below.
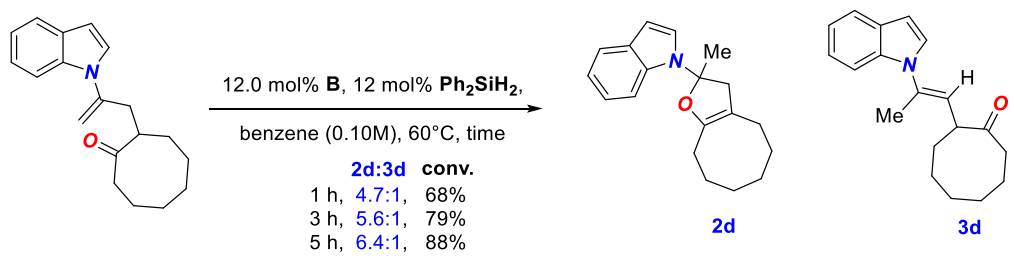

C. Interconversion of products:

Those four controlled reactions were run according to the general procedure under salen$\mathrm{Co}(\mathrm{III}) /$ silane conditions. The yield of the product was determined by ${ }^{1} \mathrm{HNMR}$ of the crude reaction mixture with internal standard. 

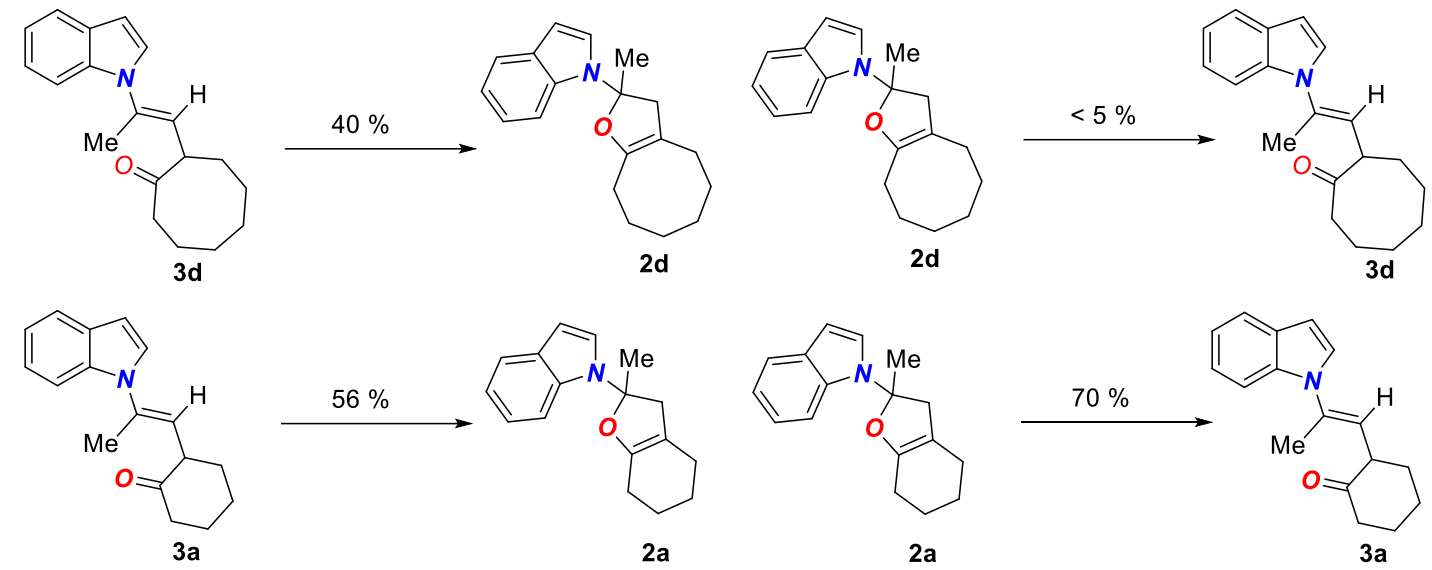

SI-33 


\section{References}

1. Yao, C.; Dahmen, T.; Gansäuer, A.; Norton, J. Anti-Markovnikov alcohols via epoxide hydrogenation through cooperative catalysis. Science 2019, 364, 764-767.

2. Li, G.; Estes, D. P.; Norton, J. R.; Ruccolo, S.; Sattler, A.; Sattler, W. Dihydrogen Activation by Cobaloximes with Various Axial Ligands. Inorg. Chem. 2014, 53, 10743-10747.

3. Floriani, C.; Puppis, M.; Calderazzo, F. N, N'-Ethylenebis (salicylideneiminato) cobalt (II). Reduction, oxidation and conversion into alkyl and acyl derivatives. J. Organomet. Chem. 1968, 12, 209-223.

4. Schaus, S. E.; Brandes, B. D.; Larrow, J. F.; Tokunaga, M.; Hansen, K. B.; Gould, A. E.; Furrow, M. E.; Jacobsen, E. N. Highly selective hydrolytic kinetic resolution of terminal epoxides catalyzed by chiral (salen) CoIII complexes. Practical synthesis of enantioenriched terminal epoxides and 1, 2-diols. J. Am. Chem. Soc. 2002, 124, 1307-1315.

5. Tanyeli, C.; Akhmedov, İ. M.; Yazıcıŏlu, E. Y. One-pot synthesis of N-substituted 2methyl-4, 5, 6, 7-tetrahydroindole derivatives. Tetrahedron Lett. 2004, 45, 9627-9629.

6. Pan, Y.; Lu, H.; Fang, Y.; Fang, X.; Chen, L.; Qian, J.; Wang, J.; Li, C. Synthesis of pyrroles via copper-catalyzed coupling of amines with bromoenones. Synthesis 2007, 2007, 12421246.

7. Li, H.; Boonnak, N.; Padwa, A. N-Alkenyl Indoles as Useful Intermediates for Alkaloid Synthesis. J. Org. Chem. 2011, 76, 9488-9496. 


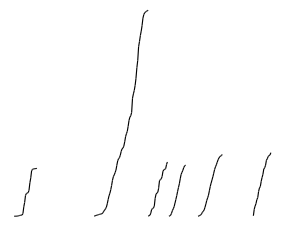

${ }^{1} \mathrm{H}$ NMR $\left(400 \mathrm{MHz}, \mathrm{CDCl}_{3}\right)$
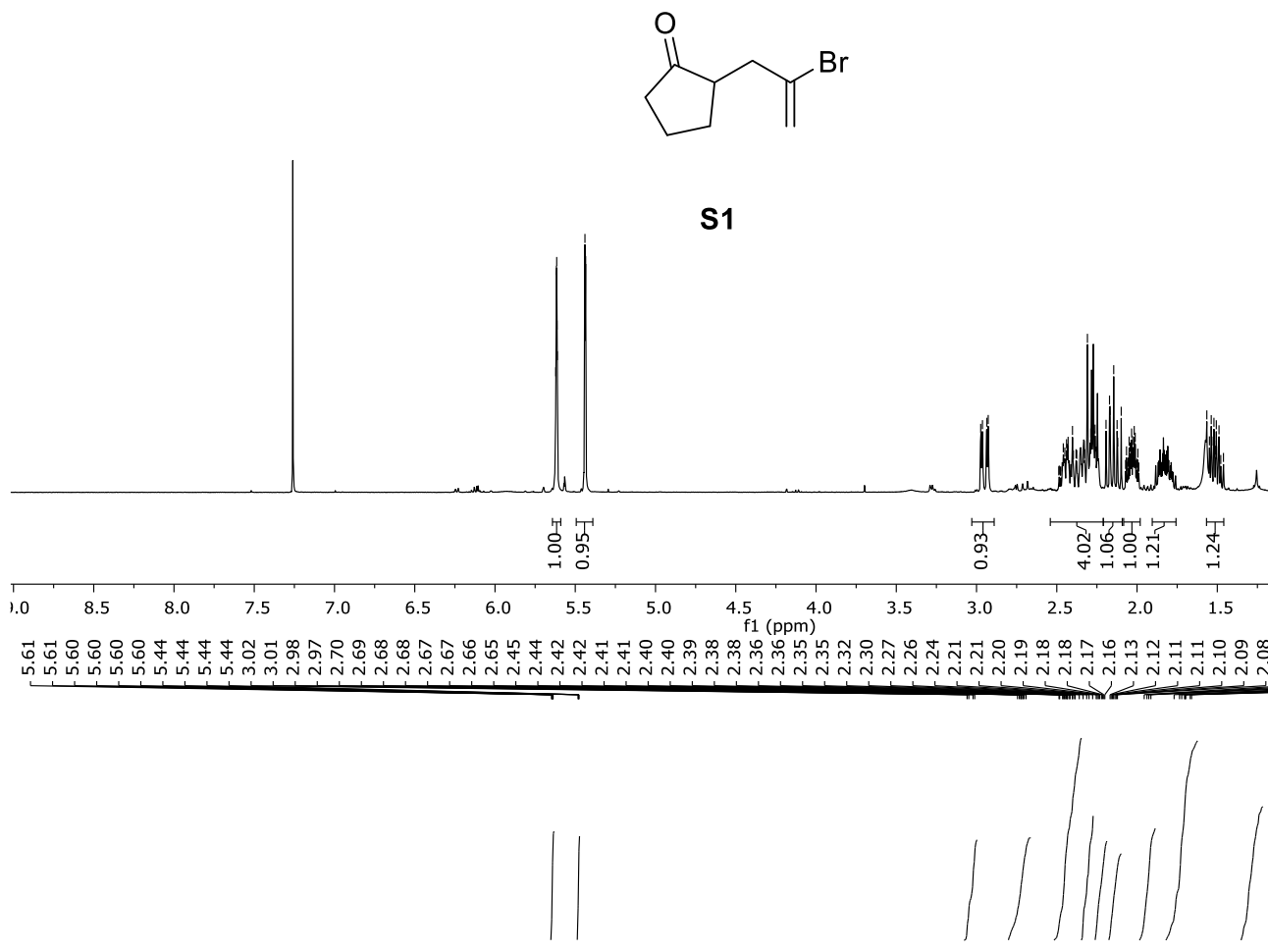

${ }^{1} \mathrm{H}$ NMR $\left(400 \mathrm{MHz}, \mathrm{CDCl}_{3}\right)$<smiles>C=C(Br)CC1CCCCC1=O</smiles>

S2

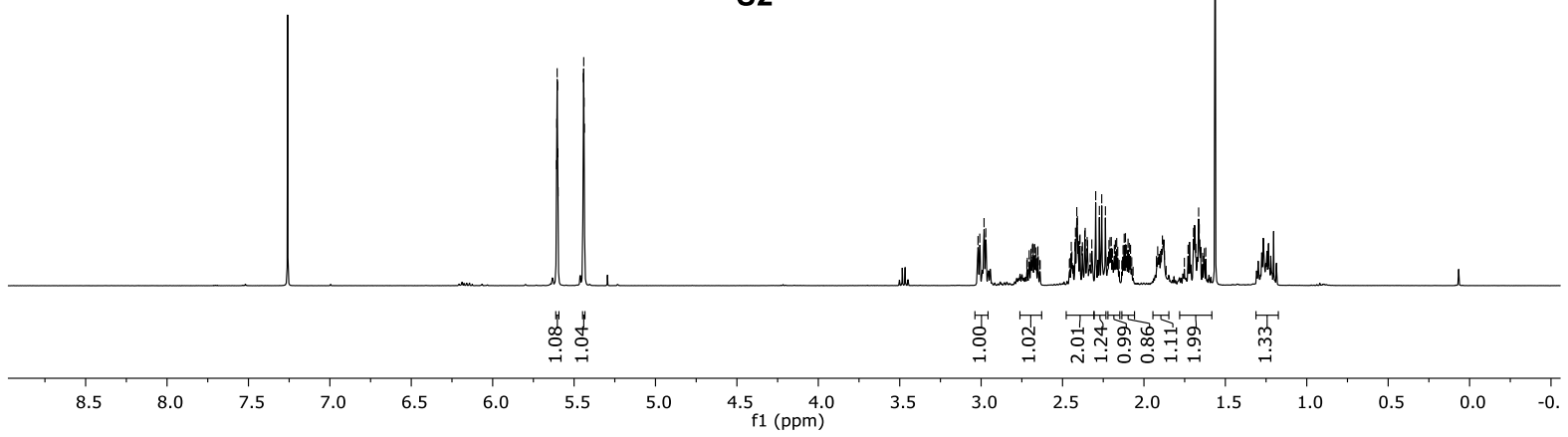

SI-35 
${ }^{1} \mathrm{H}$ NMR $\left(400 \mathrm{MHz}, \mathrm{CDCl}_{3}\right)$<smiles>C=C(Br)CC1CCCCCC1=O</smiles>

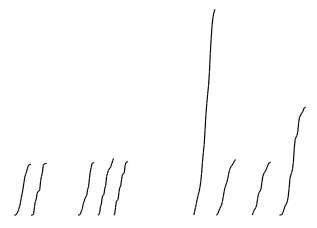

S3

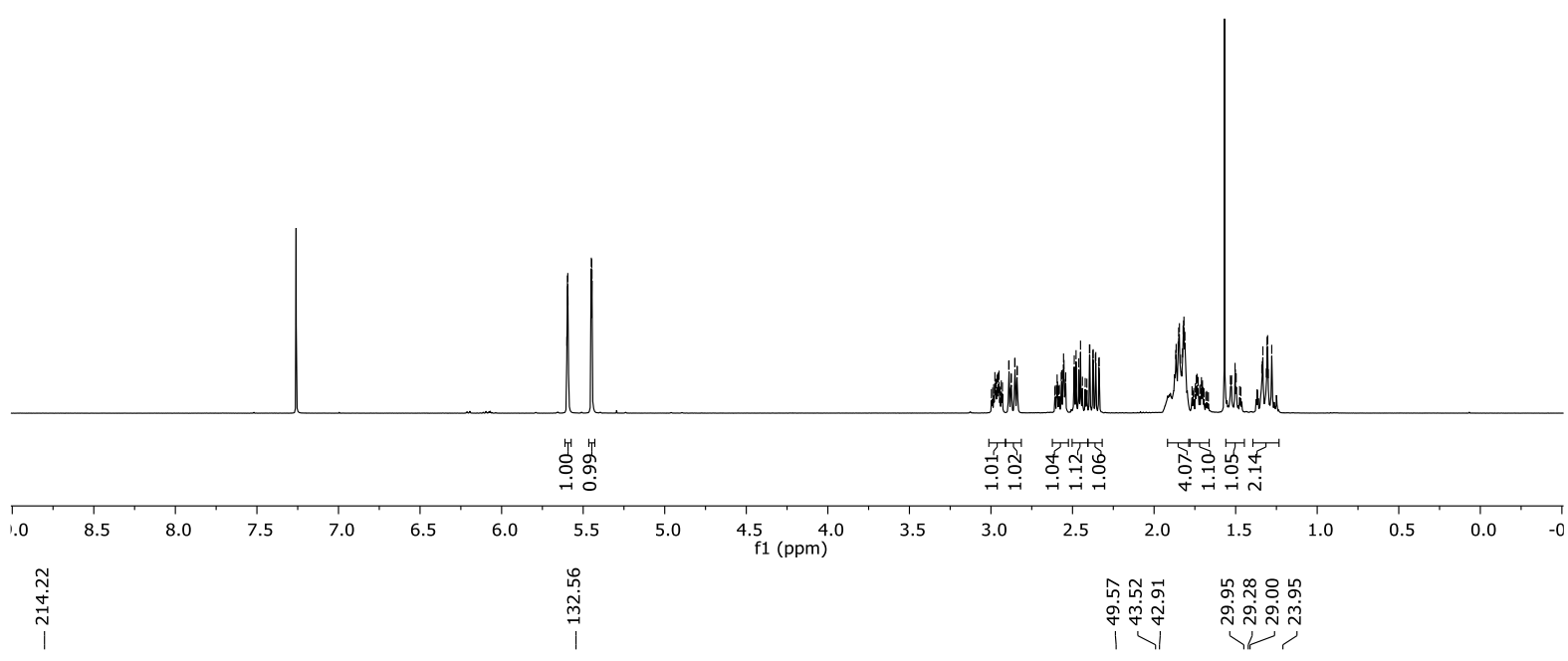

${ }^{13} \mathrm{C}$ NMR $\left(101 \mathrm{MHz}, \mathrm{CDCl}_{3}\right)$<smiles>C=C(Br)CC1CCCCCC1=O</smiles>

S3
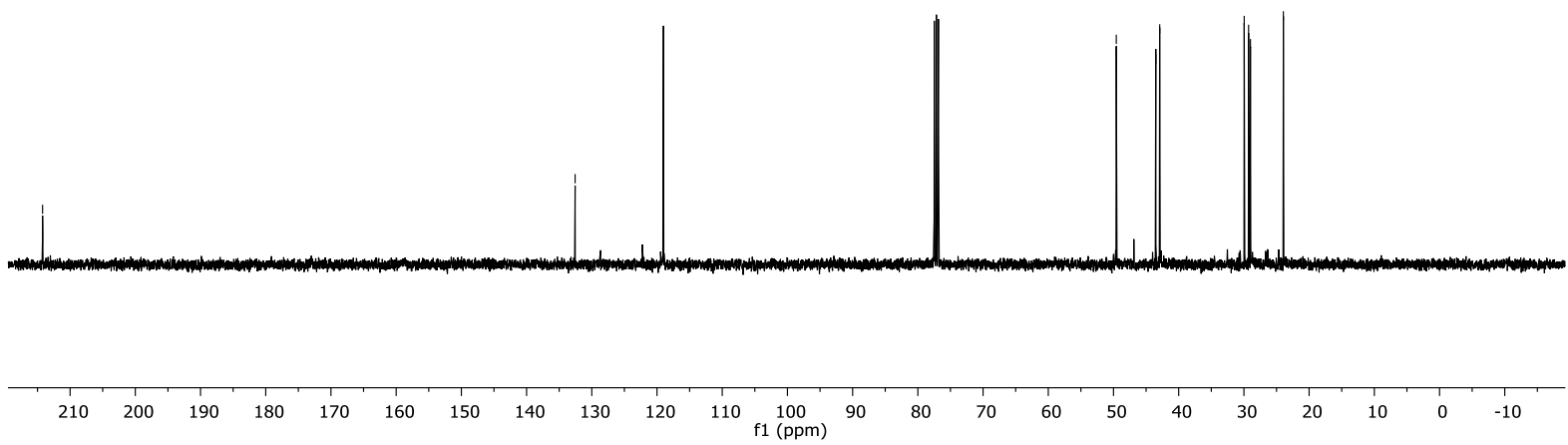

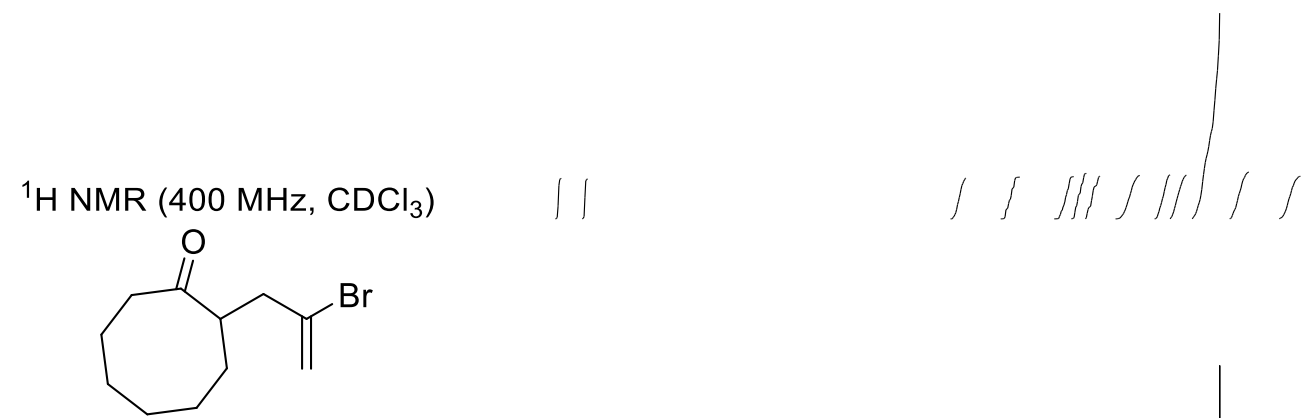

S4

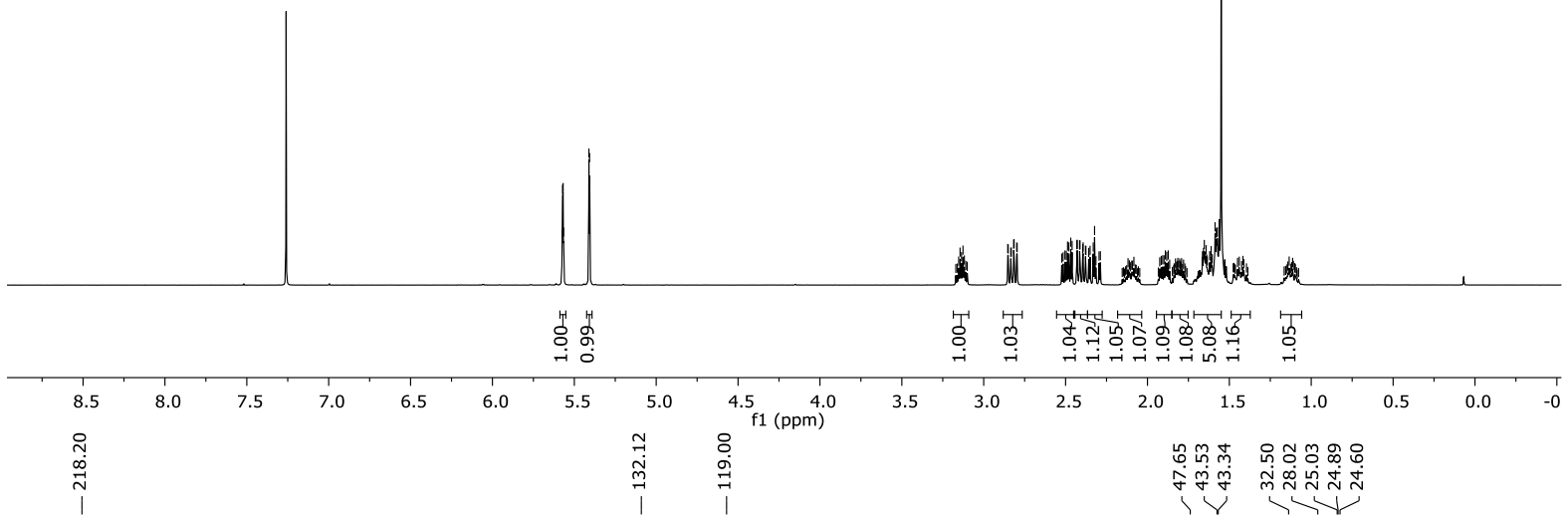

${ }^{13} \mathrm{C}$ NMR $\left(101 \mathrm{MHz}, \mathrm{CDCl}_{3}\right)$<smiles>C=C(Br)CC1CCCCCCC1=O</smiles>

S4
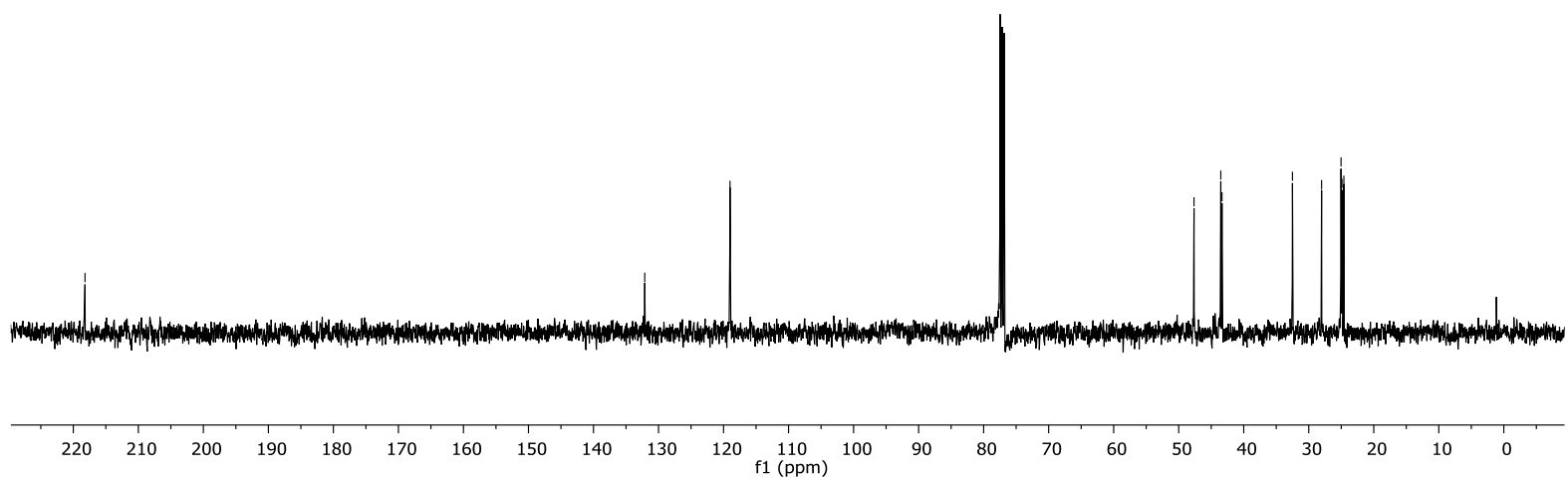
${ }^{1} \mathrm{H}$ NMR $\left(400 \mathrm{MHz}, \mathrm{CDCl}_{3}\right)$<smiles>C=C(Br)CC1CCCCCCCCCCC1=O</smiles>

S5

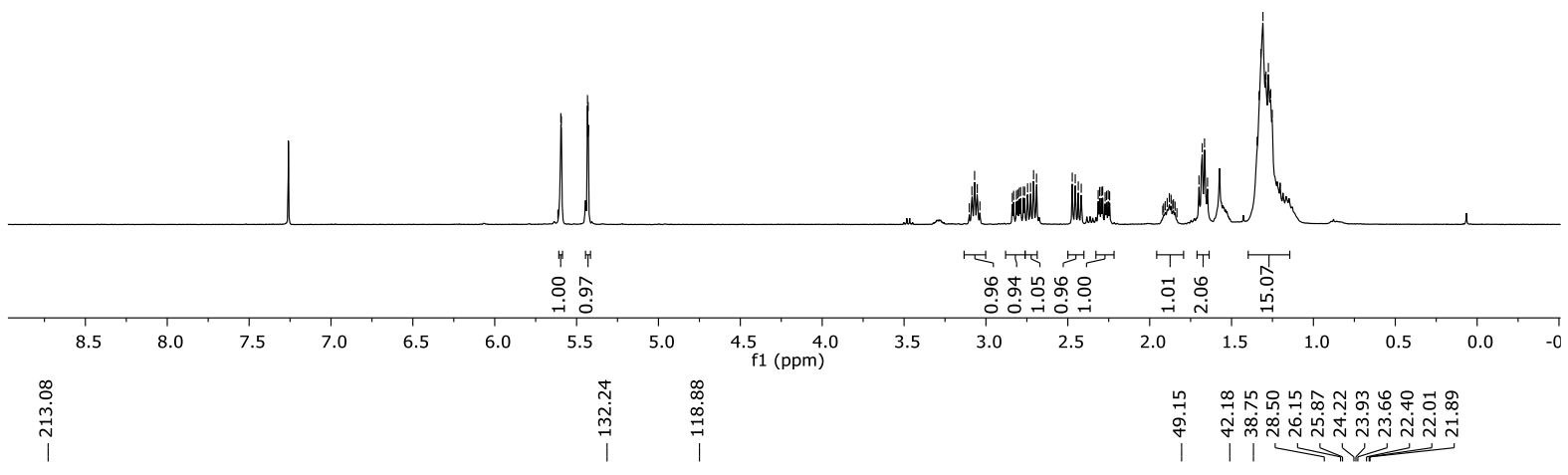

${ }^{13} \mathrm{C}$ NMR $\left(101 \mathrm{MHz}, \mathrm{CDCl}_{3}\right)$<smiles>C=C(Br)CC1CCCCCCCCCCC1=O</smiles>

S5
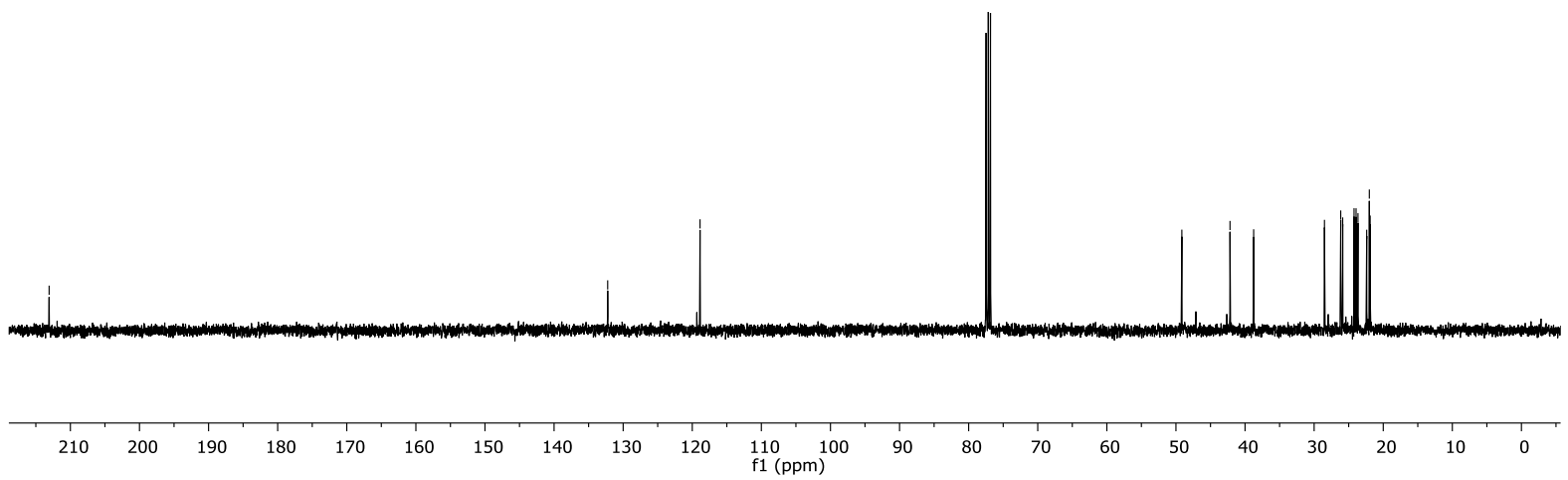

SI-38 


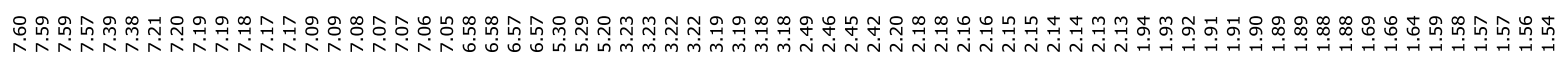

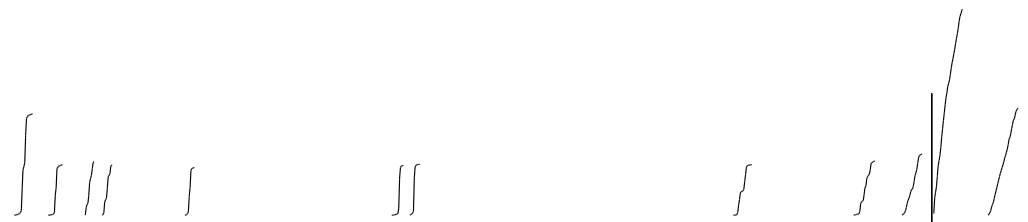

${ }^{1} \mathrm{H}$ NMR $\left(400 \mathrm{MHz}, \mathrm{CD}_{3} \mathrm{C}(\mathrm{O}) \mathrm{CD}_{3}\right)$

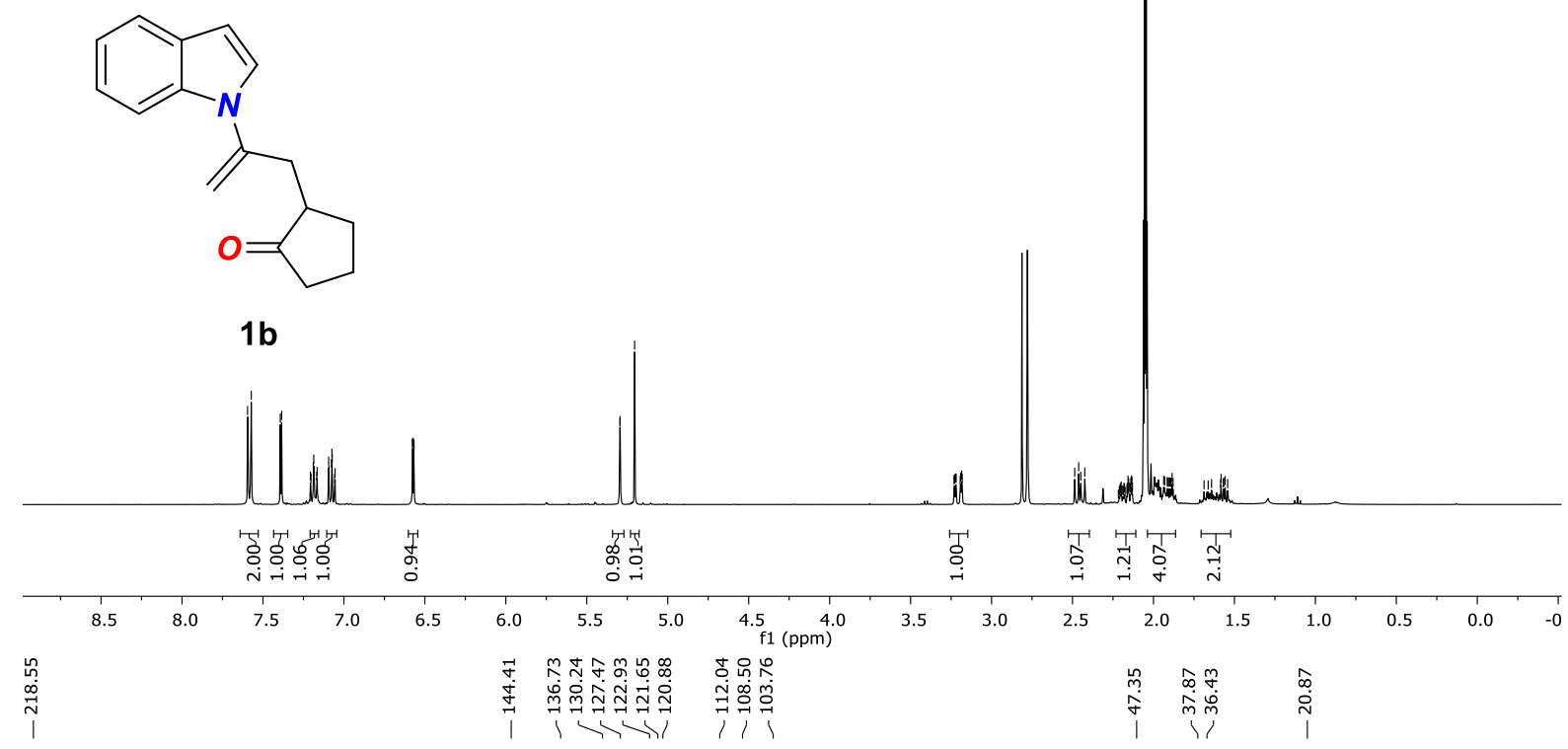

${ }^{13} \mathrm{C}$ NMR $\left(101 \mathrm{MHz}, \mathrm{CD}_{3} \mathrm{C}(\mathrm{O}) \mathrm{CD}_{3}\right)$
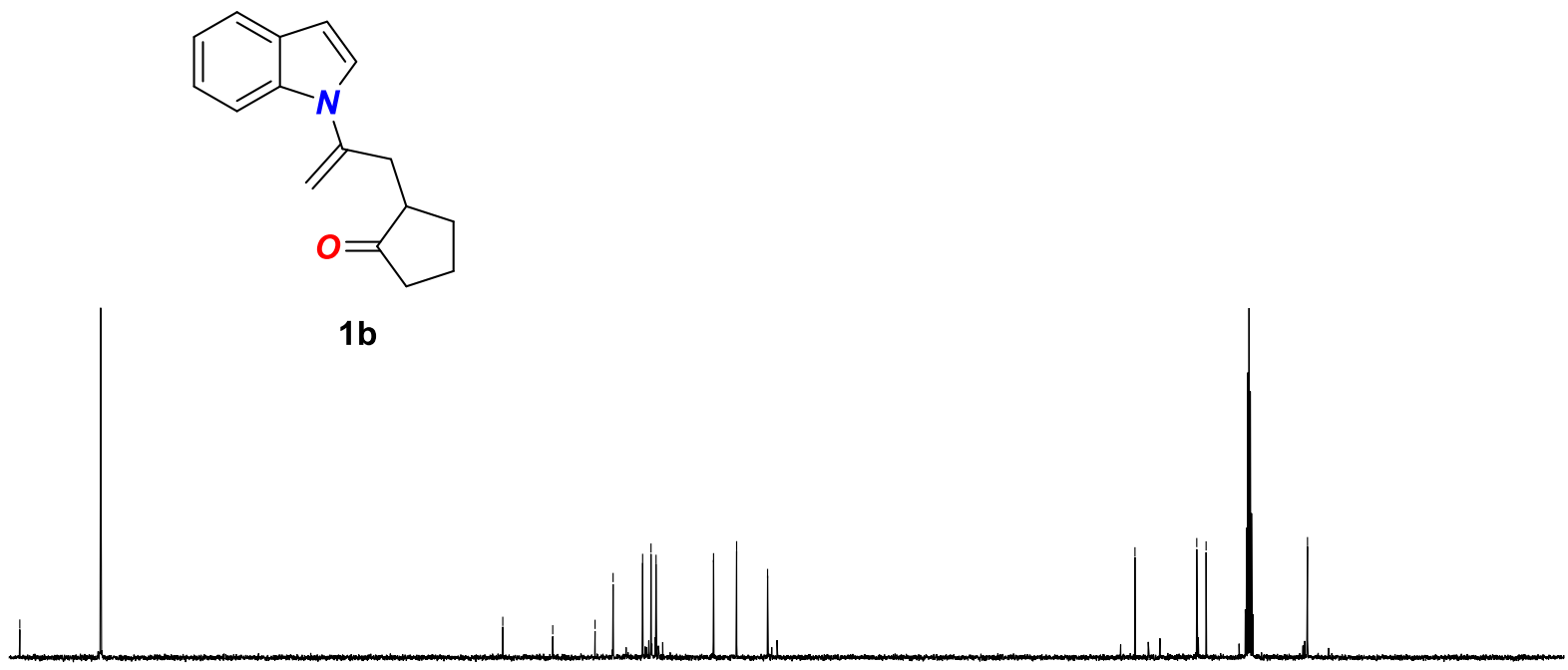

\begin{tabular}{llllllllllllllllllllllll}
\hline 20 & 210 & 200 & 190 & 180 & 170 & 160 & 150 & 140 & 130 & 120 & 110 & 100 & 90 & 80 & 70 & 60 & 50 & 40 & 30 & 20 & 10 & 0 & -10
\end{tabular} 


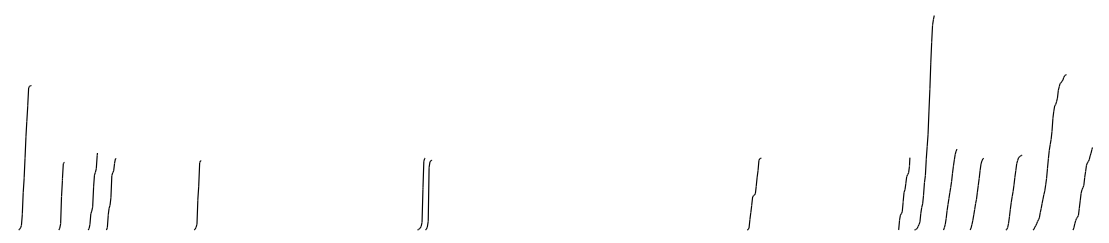

${ }^{1} \mathrm{H}$ NMR $\left(400 \mathrm{MHz}, \mathrm{CD}_{3} \mathrm{C}(\mathrm{O}) \mathrm{CD}_{3}\right)$

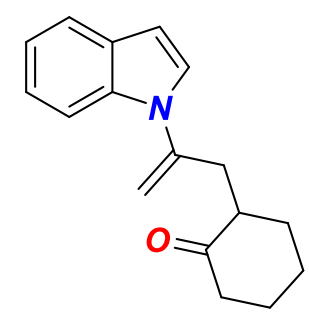

$1 \mathrm{a}$

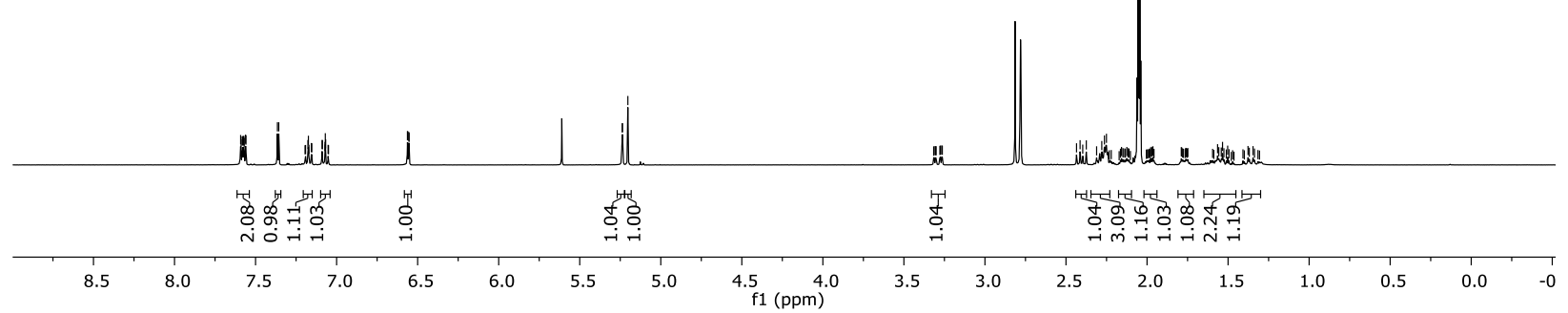




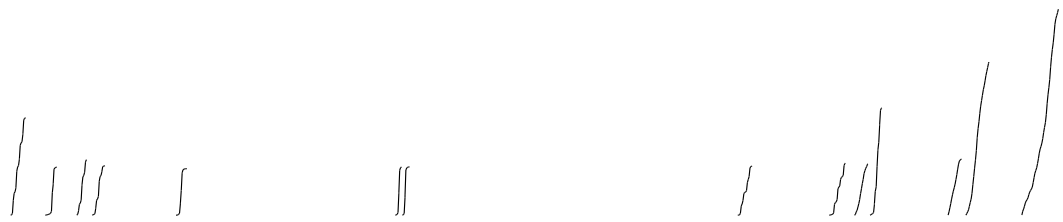

${ }^{1} \mathrm{H}$ NMR $\left(400 \mathrm{MHz}, \mathrm{CD}_{3} \mathrm{C}(\mathrm{O}) \mathrm{CD}_{3}\right)$

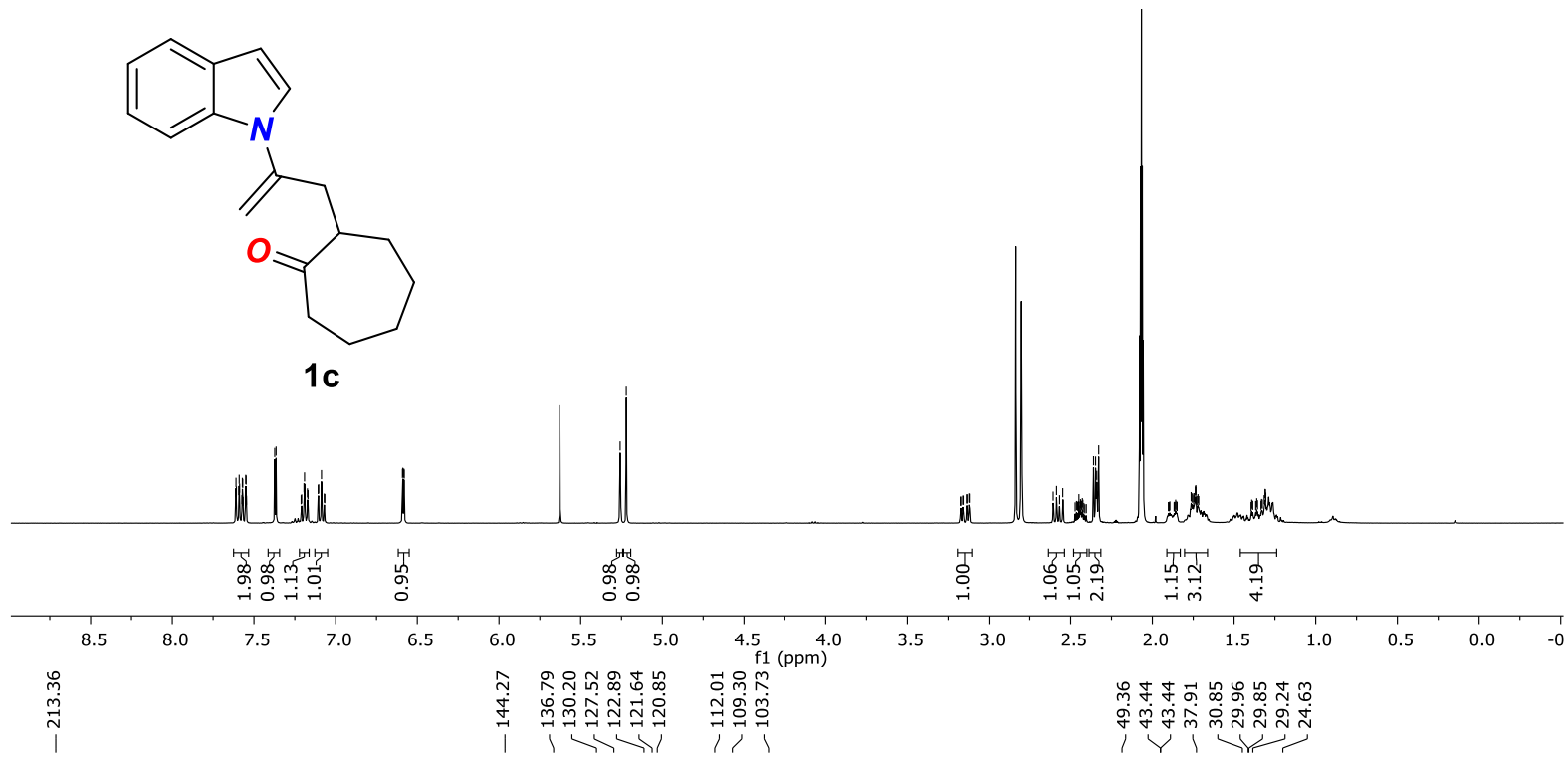

${ }^{13} \mathrm{C}$ NMR $\left(101 \mathrm{MHz}, \mathrm{CD}_{3} \mathrm{C}(\mathrm{O}) \mathrm{CD}_{3}\right)$

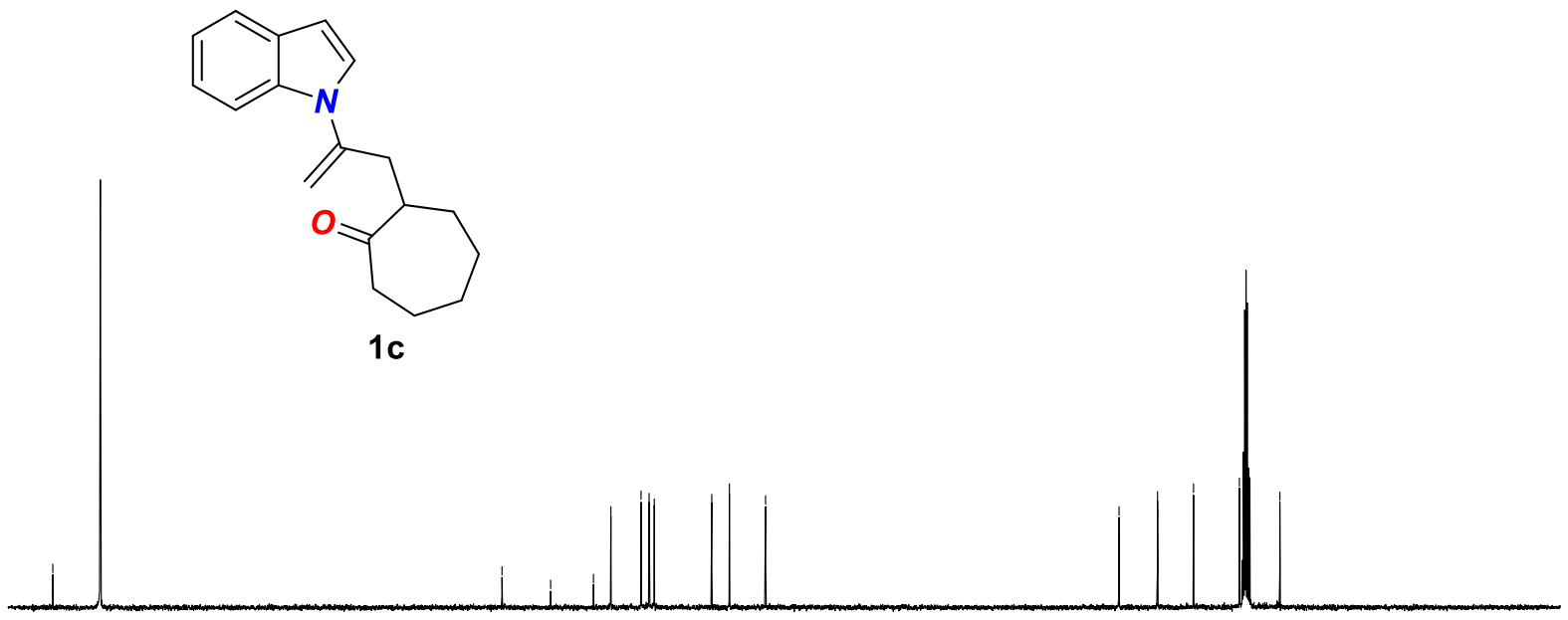

\begin{tabular}{llllllllllllllllllllllllllllll}
\hline 20 & 210 & 200 & 190 & 180 & 170 & 160 & 150 & 140 & 130 & 120 & 110 & 100 & 90 & 80 & 70 & 60 & 50 & 40 & 30 & 20 & 10 & 0 & -10
\end{tabular} 

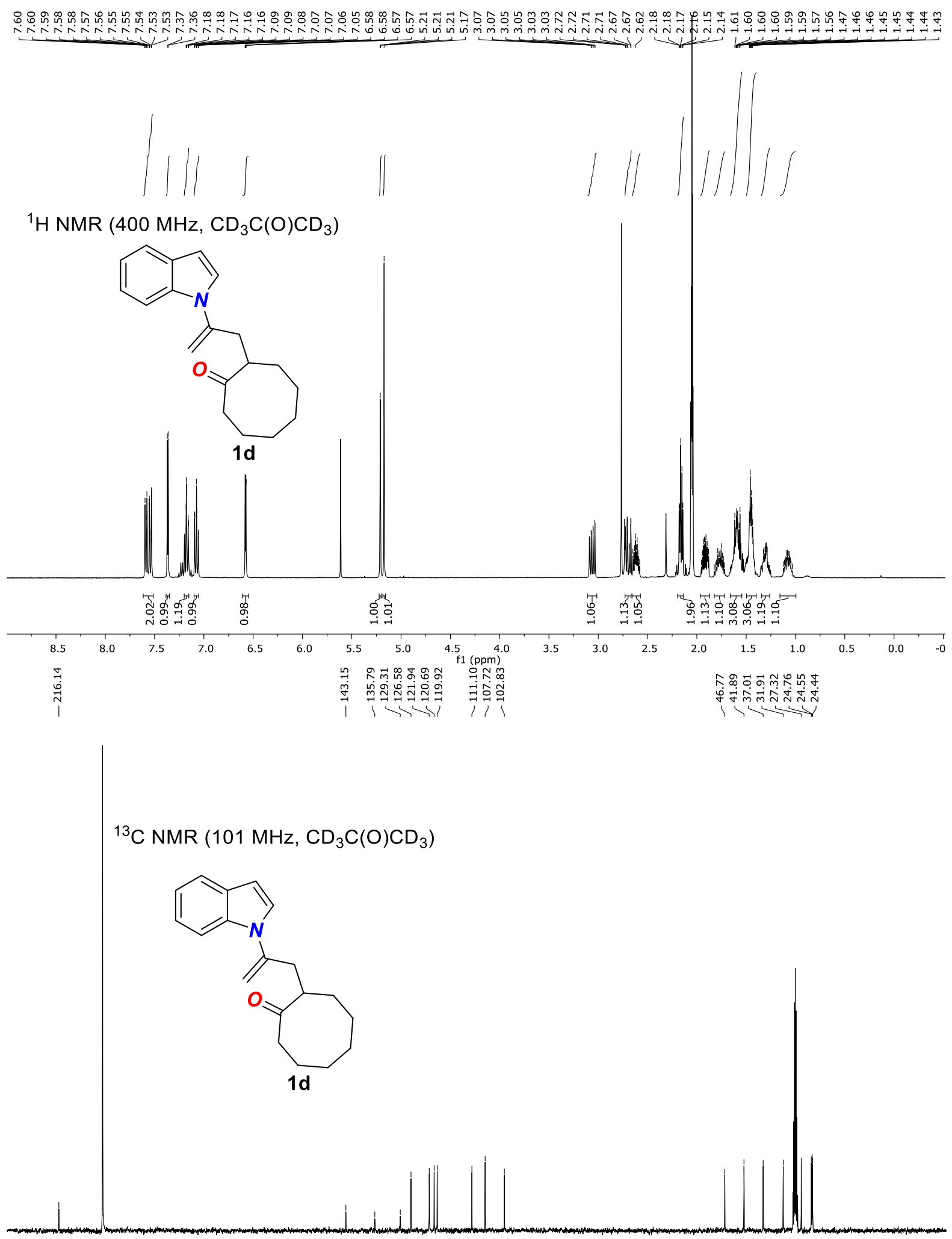

$\begin{array}{llllllllllllllllllllllllllllllllllll}220 & 210 & 200 & 190 & 180 & 170 & 160 & 150 & 140 & 130 & 120 & 110 & 100 & 90 & 80 & 70 & 60 & 50 & 40 & 30 & 20 & 10 & 0 & 0\end{array}$ 
${ }^{1} \mathrm{H}$ NMR $\left(400 \mathrm{MHz}, \mathrm{CD}_{3} \mathrm{C}(\mathrm{O}) \mathrm{CD}_{3}\right)$

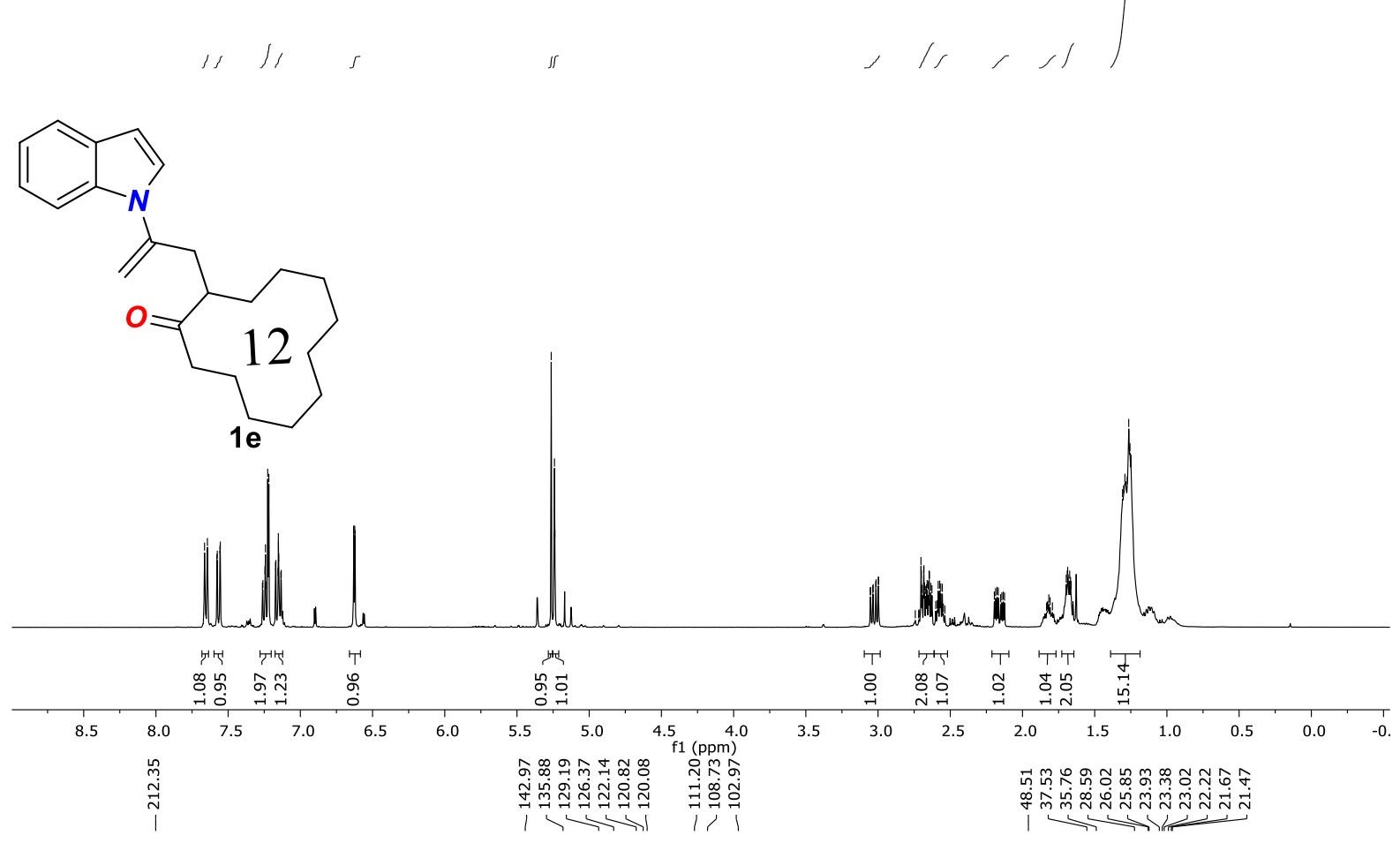

${ }^{13} \mathrm{C}$ NMR $\left(101 \mathrm{MHz}, \mathrm{CD}_{3} \mathrm{C}(\mathrm{O}) \mathrm{CD}_{3}\right)$
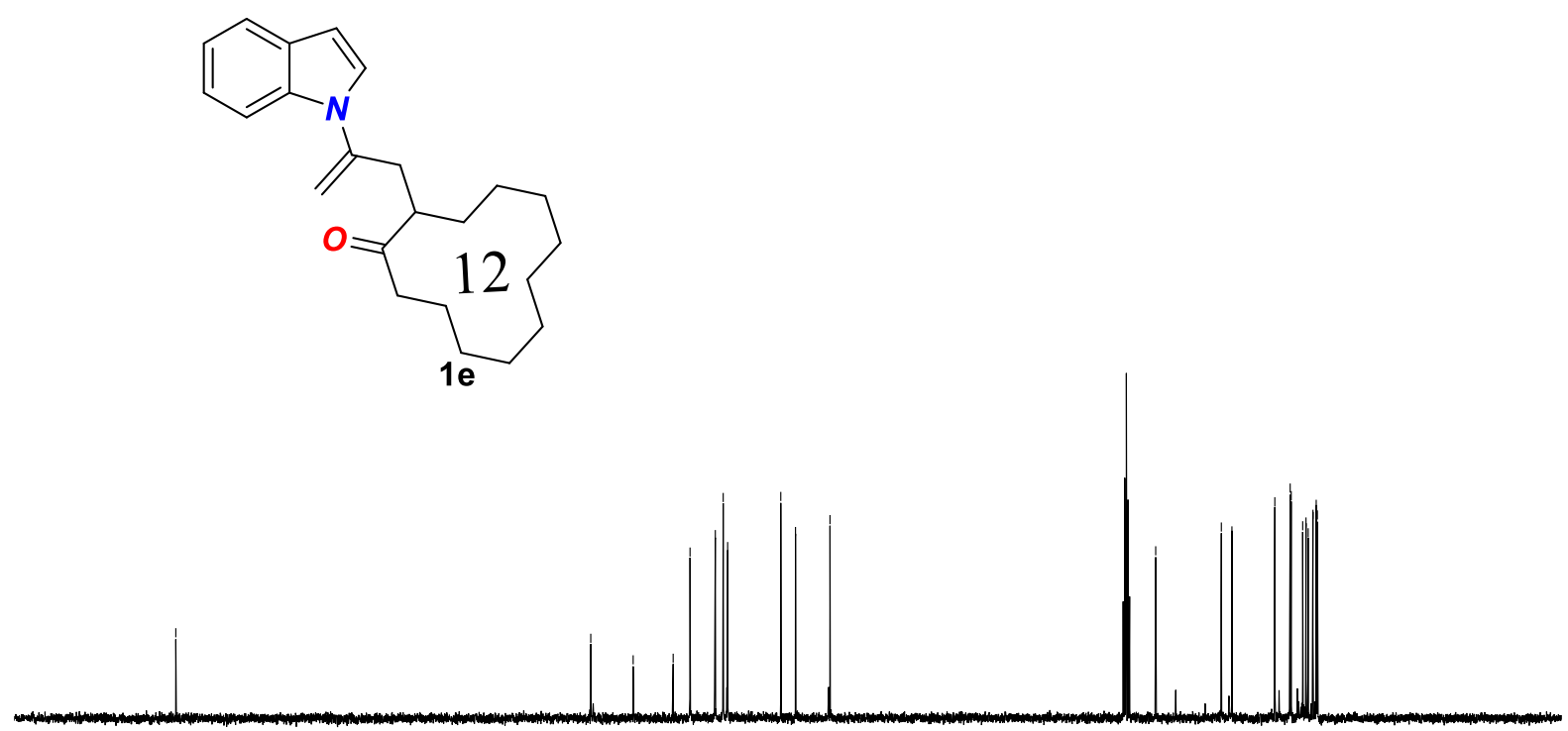

$\begin{array}{lllllllllllllllllllllllllllll}230 & 220 & 210 & 200 & 190 & 180 & 170 & 160 & 150 & 140 & 130 & 120 & 110 & 100 & 90 & 80 & 70 & 60 & 50 & 40 & 30 & 20 & 10 & 0 & -10\end{array}$ 


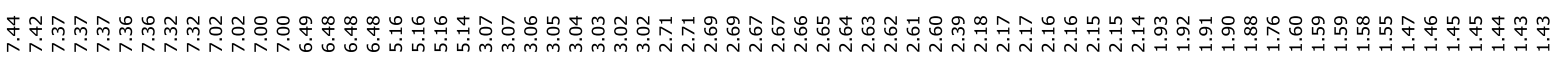

${ }^{1} \mathrm{H}$ NMR $\left(400 \mathrm{MHz}, \mathrm{CD}_{3} \mathrm{C}(\mathrm{O}) \mathrm{CD}_{3}\right)$<smiles>C#CCCC=C</smiles><smiles>C=C</smiles>

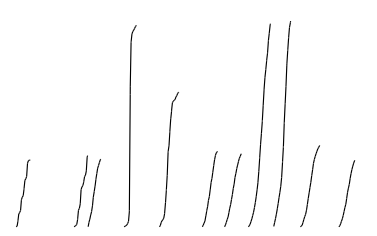

Me

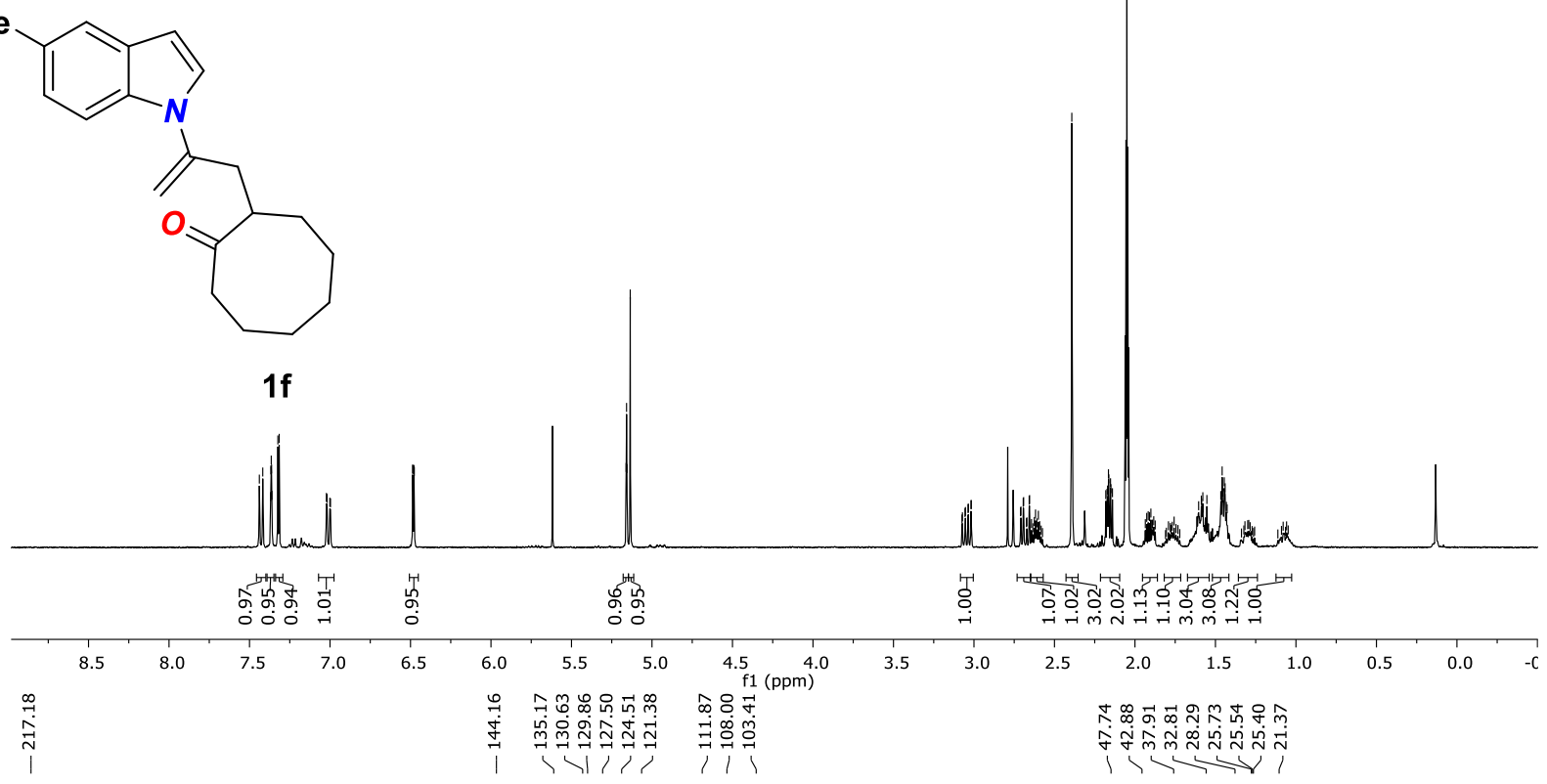

${ }^{13} \mathrm{C}$ NMR $\left(101 \mathrm{MHz}, \mathrm{CD}_{3} \mathrm{C}(\mathrm{O}) \mathrm{CD}_{3}\right)$

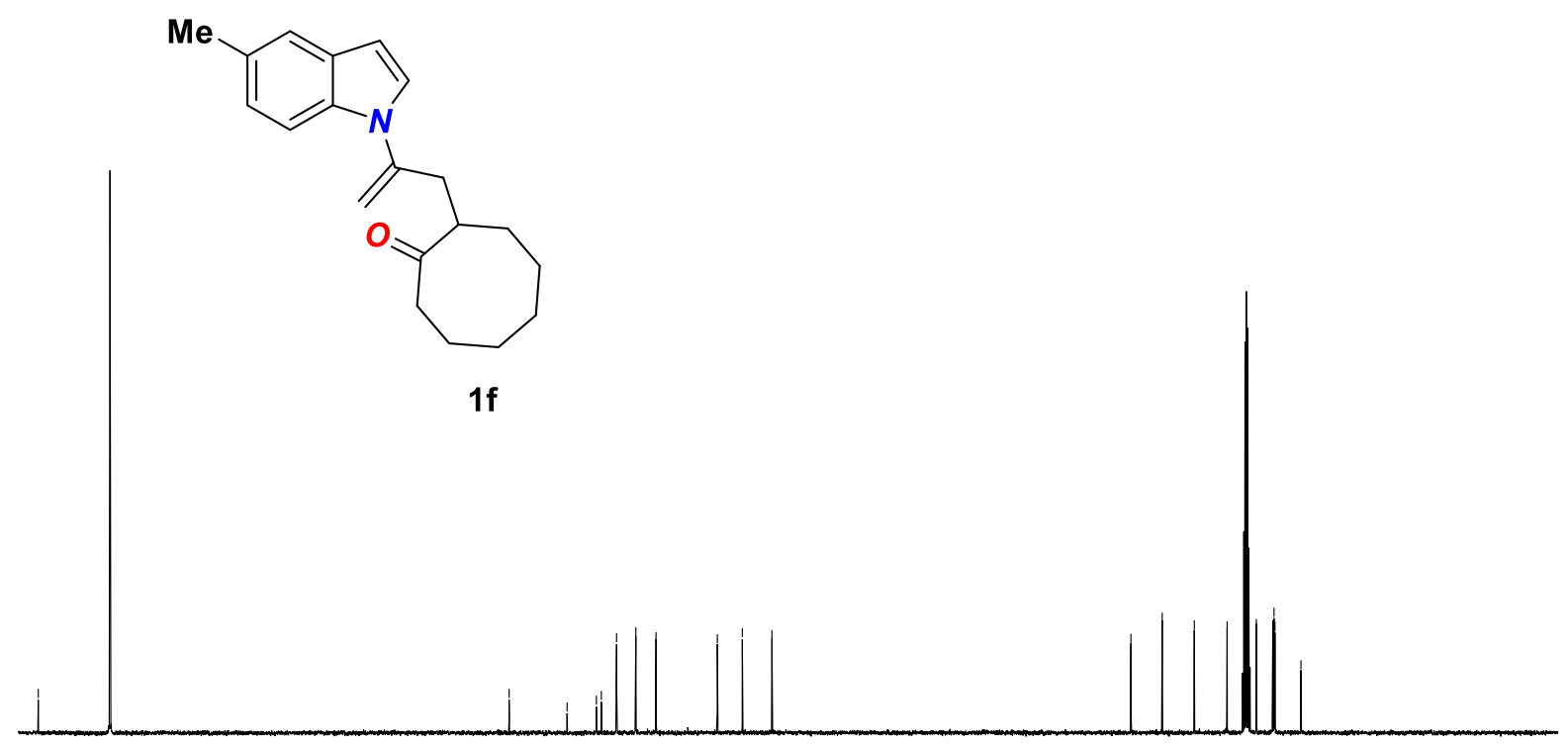

$\begin{array}{llllllllllllllllllllllllllllllllllll}20 & 210 & 200 & 190 & 180 & 170 & 160 & 150 & 140 & 130 & 120 & 110 & 100 & 90 & 80 & 70 & 60 & 50 & 40 & 30 & 20 & 10 & 0 & -10 & \end{array}$ 
${ }^{1} \mathrm{H}$ NMR $\left(400 \mathrm{MHz}, \mathrm{CD}_{2} \mathrm{Cl}_{2}\right)$

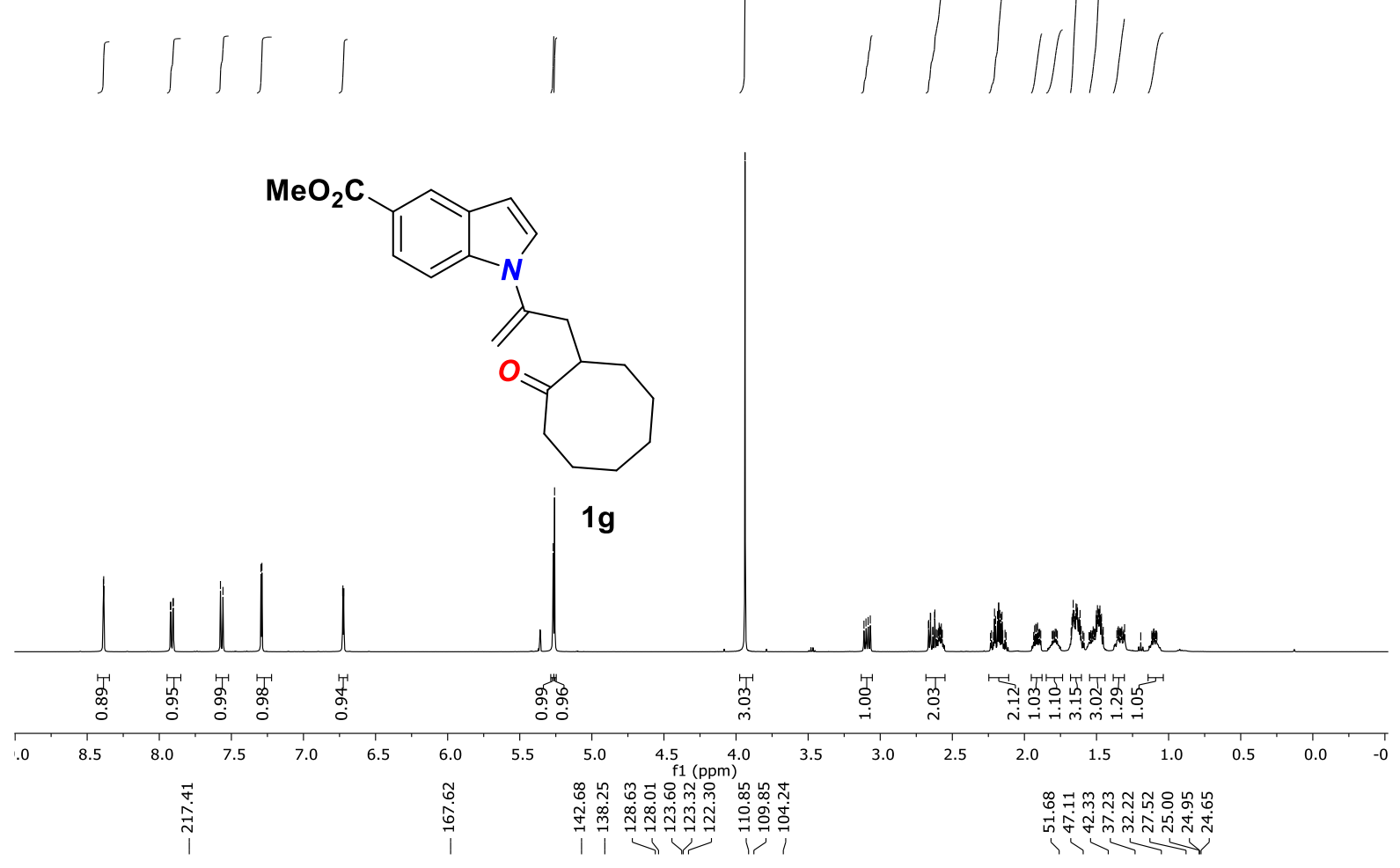

${ }^{13} \mathrm{C}$ NMR $\left(101 \mathrm{MHz}, \mathrm{CD}_{2} \mathrm{Cl}_{2}\right)$

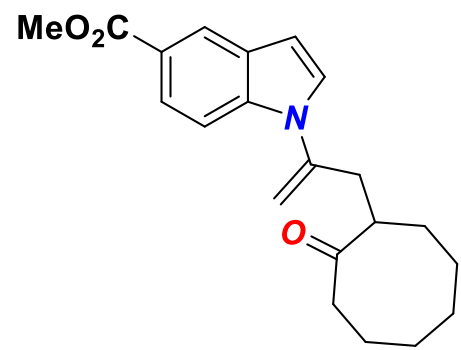

1g

$\begin{array}{lllllllllllllllllllllllllllllll}150 & 240 & 230 & 220 & 210 & 200 & 190 & 180 & 170 & 160 & 150 & 140 & 130 & 120 & 110 & 100 & 90 & 80 & 70 & 60 & 50 & 40 & 30 & 20 & 10 & 0 & -1\end{array}$ 


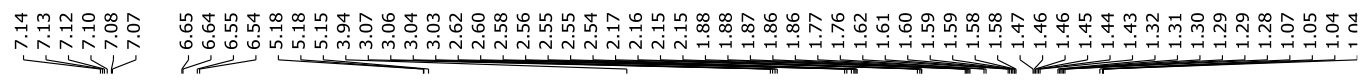

${ }^{1} \mathrm{H}$ NMR $\left(400 \mathrm{MHz}, \mathrm{CD}_{2} \mathrm{Cl}_{2}\right)$
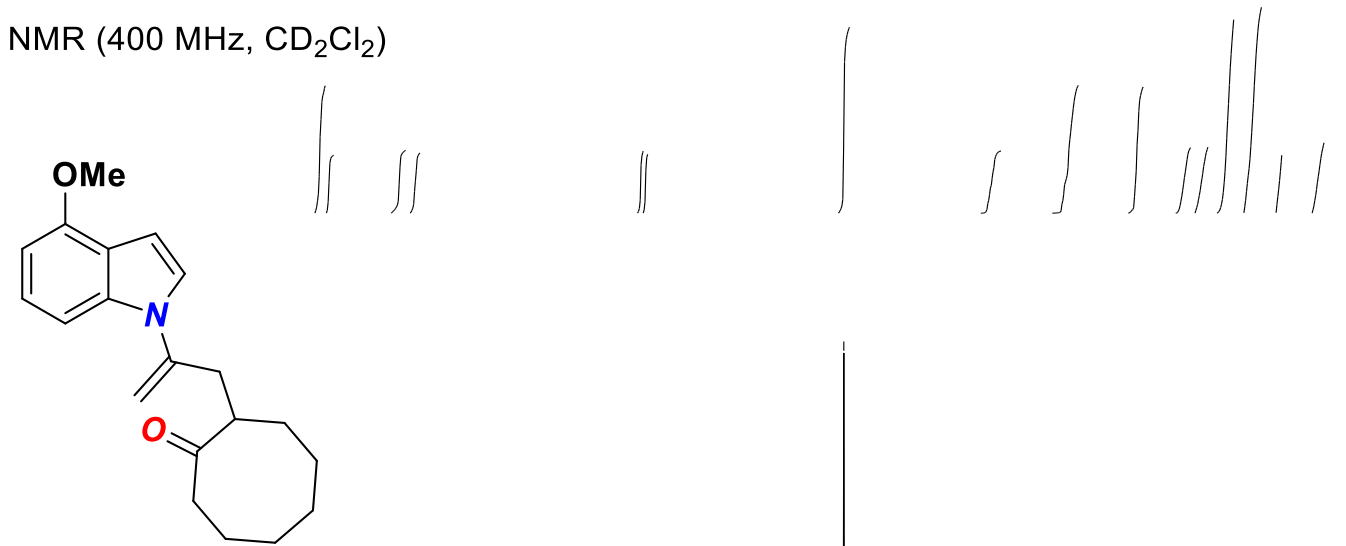

$1 \mathrm{~h}$

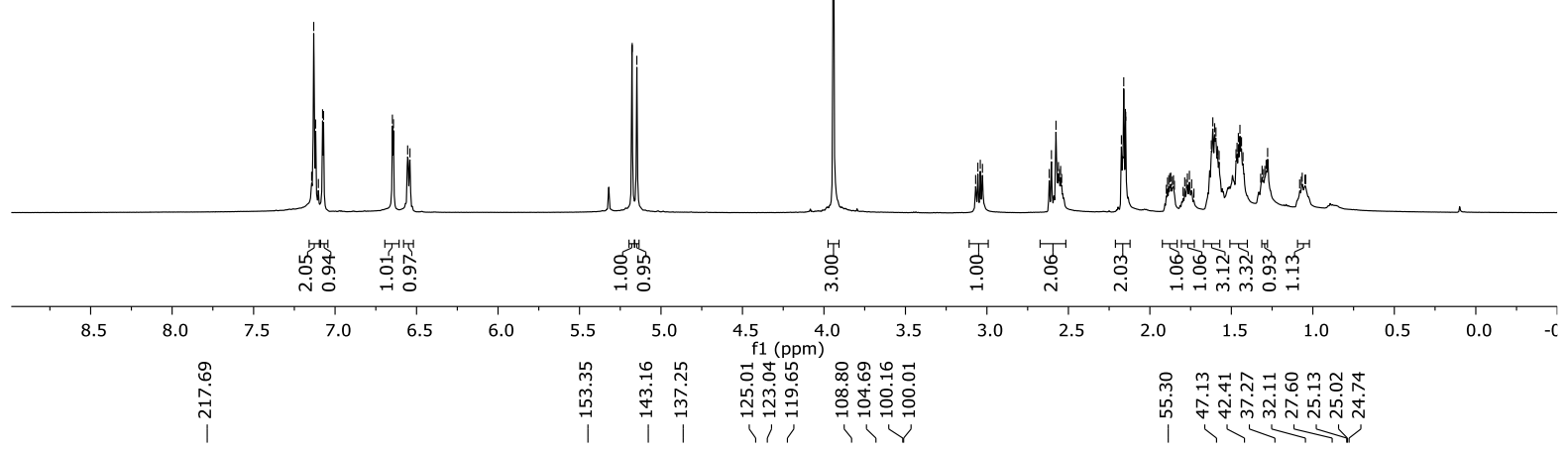

${ }^{13} \mathrm{C}$ NMR $\left(101 \mathrm{MHz}, \mathrm{CD}_{2} \mathrm{Cl}_{2}\right)$

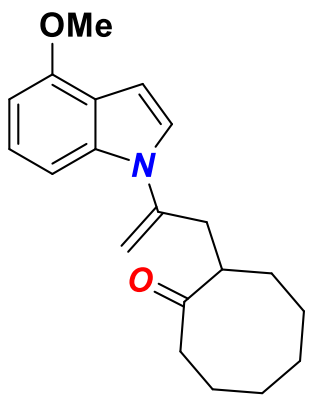

$1 \mathrm{~h}$

$\begin{array}{lllllllllllll}.50 & 240 & 230 & 220 & 210 & 200 & 190 & 180 & 170 & 160 & 150 & 140 & 130 \\ \mathrm{f} 1(\mathrm{ppm}) & 120 & 110\end{array}$ 
${ }^{1} \mathrm{H}$ NMR $\left(400 \mathrm{MHz}, \mathrm{CD}_{3} \mathrm{C}(\mathrm{O}) \mathrm{CD}_{3}\right)$<smiles>C=CCCC</smiles>
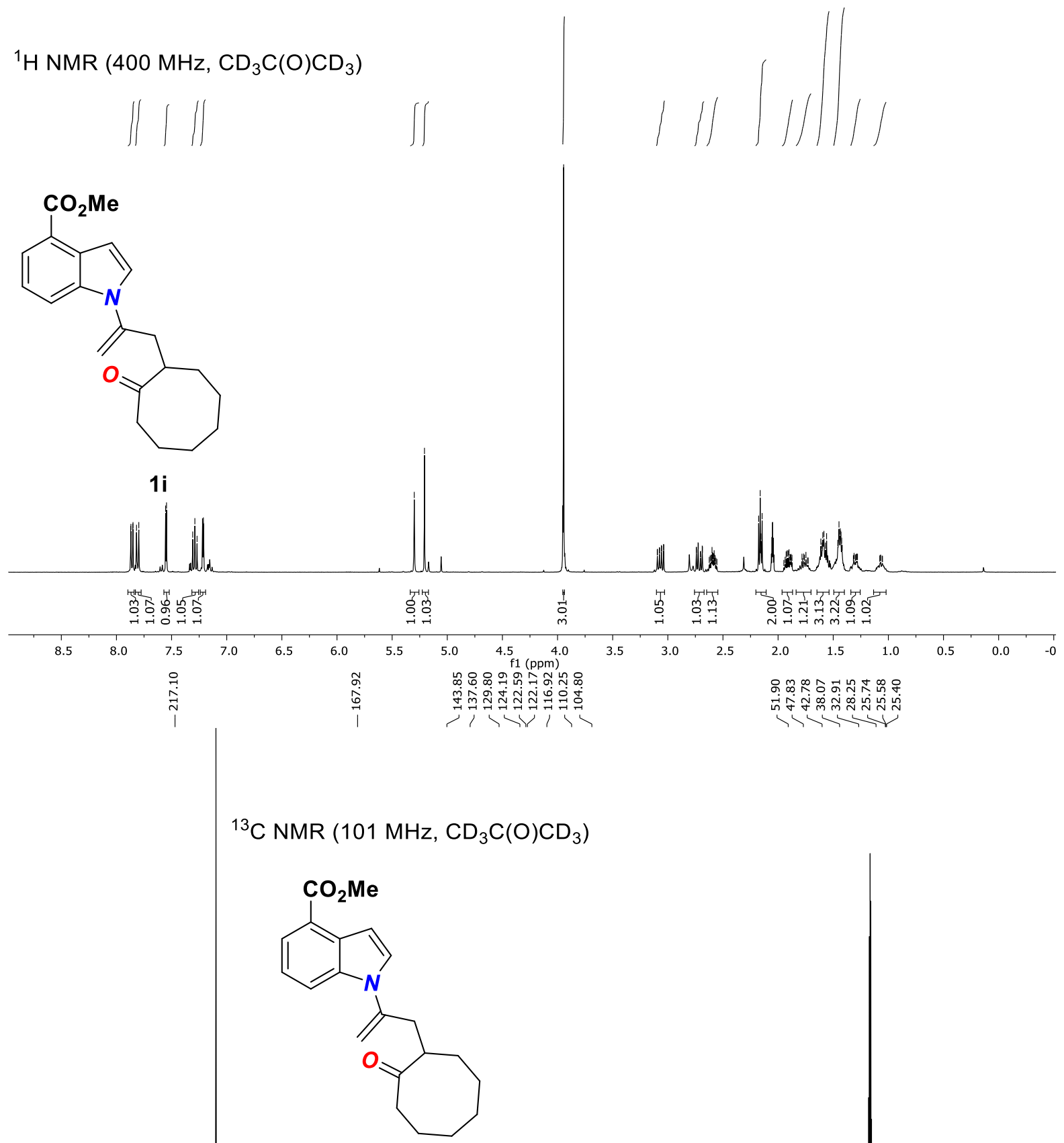

$1 \mathrm{i}$

$\begin{array}{llllllllllllllllllllllllllllllllllllllllll}260 & 250 & 240 & 230 & 220 & 210 & 200 & 190 & 180 & 170 & 160 & 150 & 140 & 130 & 120 & 110 & 100 & 90 & 80 & 70 & 60 & 50 & 40 & 30 & 20 & 10 & 0 & -10 & --4\end{array}$ 


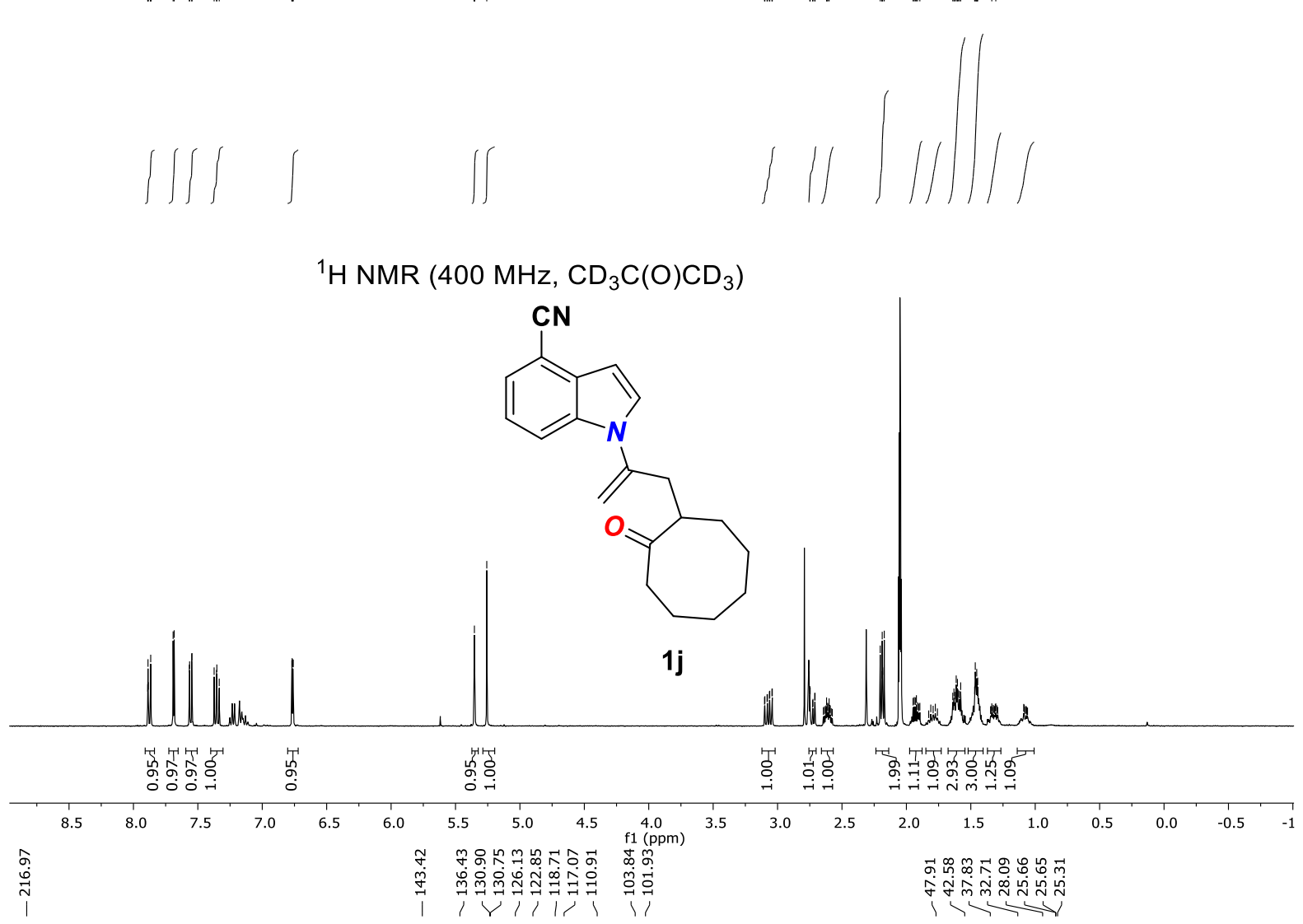

${ }^{13} \mathrm{C}$ NMR $\left(101 \mathrm{MHz}, \mathrm{CD}_{3} \mathrm{C}(\mathrm{O}) \mathrm{CD}_{3}\right)$

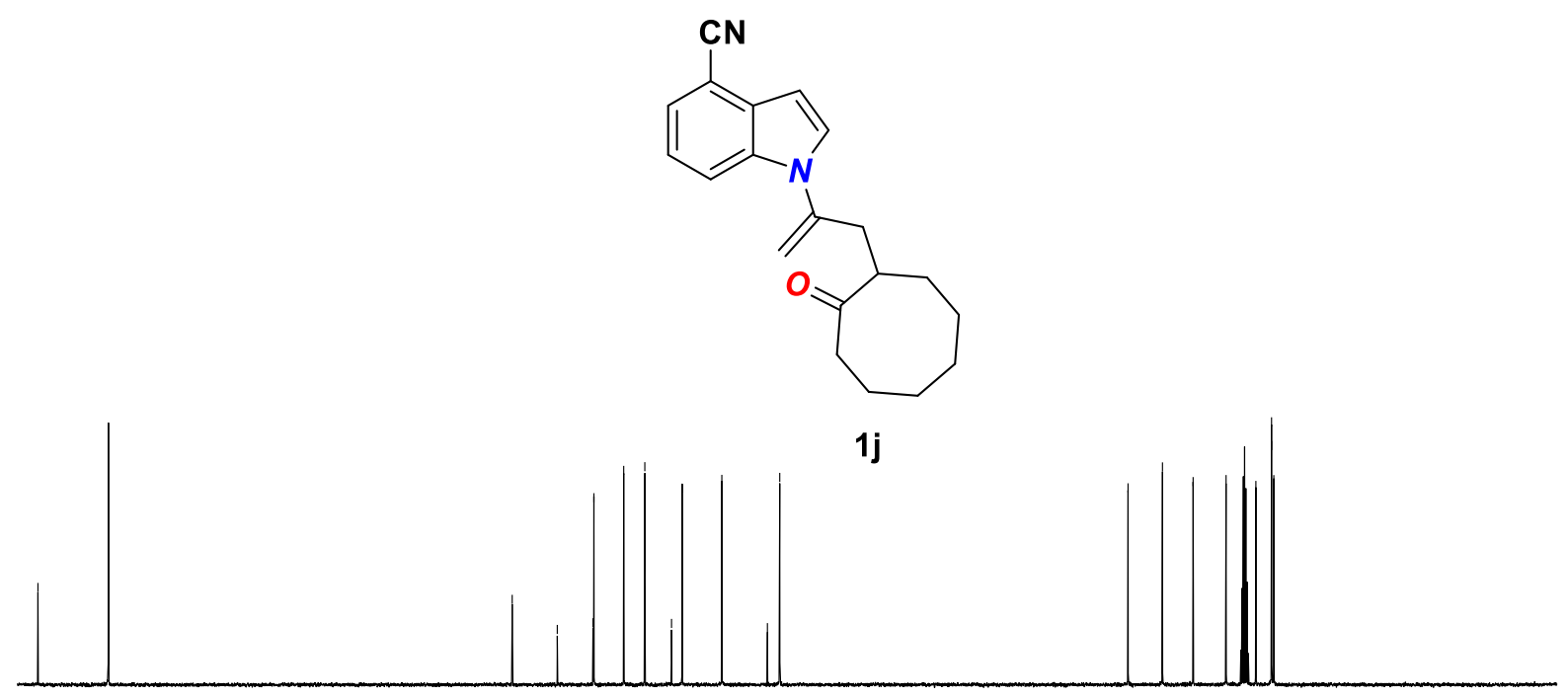

\begin{tabular}{|lllllllllllllllllllllllll}
\hline 20 & 210 & 200 & 190 & 180 & 170 & 160 & 150 & 140 & 130 & 120 & 110 & $\begin{array}{c}100 \\
\mathrm{f} 1(\mathrm{ppm})\end{array}$ & 90 & 80 & 70 & 60 & 50 & 40 & 30 & 20 & 10 & 0 & -10
\end{tabular} 


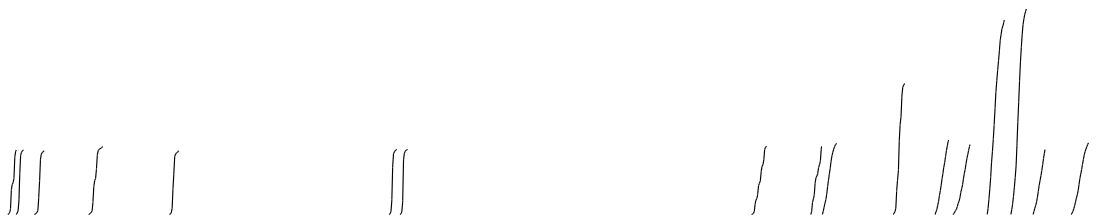

${ }^{1} \mathrm{H}$ NMR $\left(400 \mathrm{MHz}, \mathrm{CD}_{3} \mathrm{C}(\mathrm{O}) \mathrm{CD}_{3}\right)$
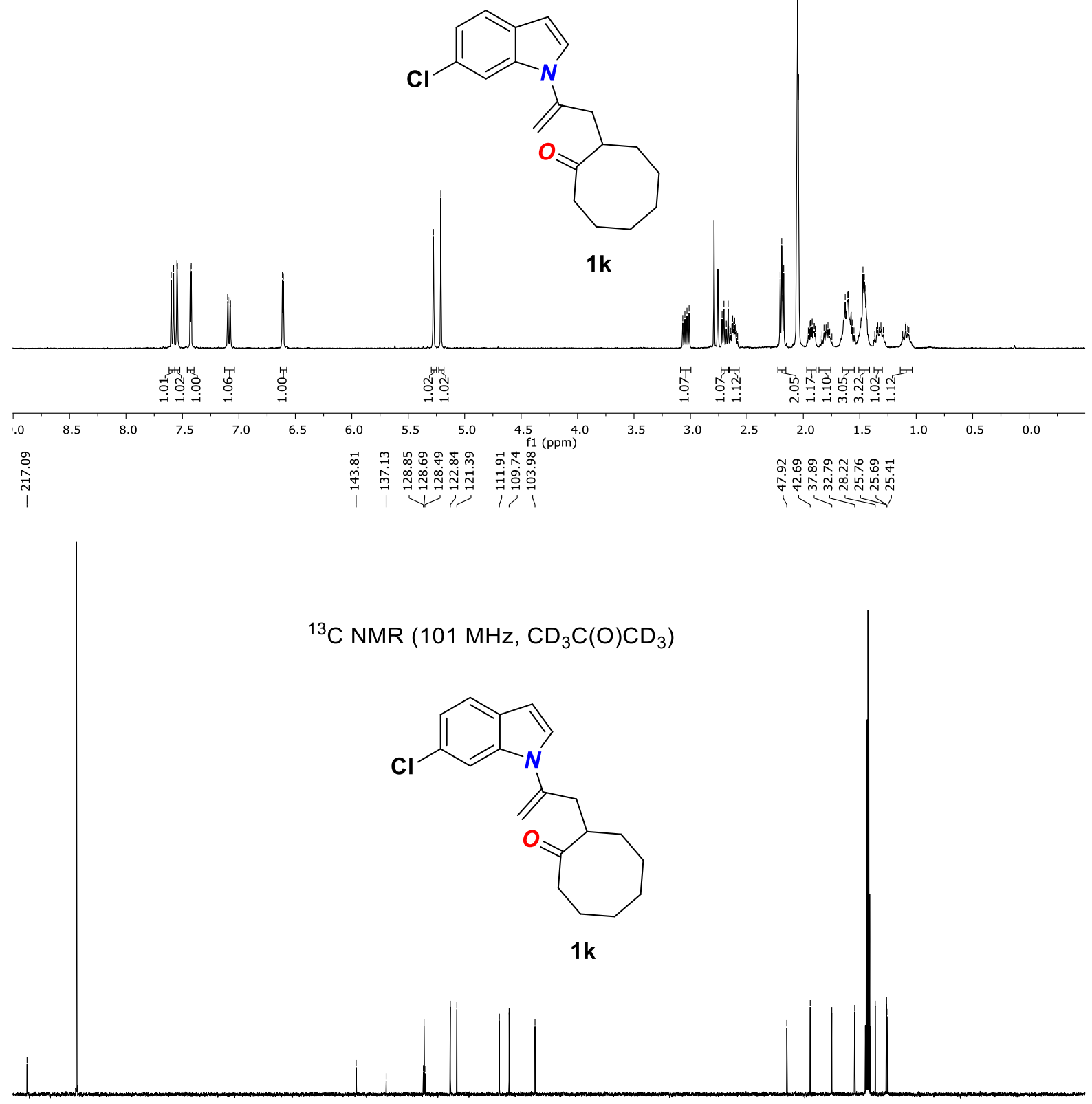

\begin{tabular}{lllllllllllllllllllllllllllllllllll}
\hline 20 & 210 & 200 & 190 & 180 & 170 & 160 & 150 & 140 & 130 & 120 & 110 & 100 & 90 & 80 & 70 & 60 & 50 & 40 & 30 & 20 & 10 & 0 & -10
\end{tabular} 


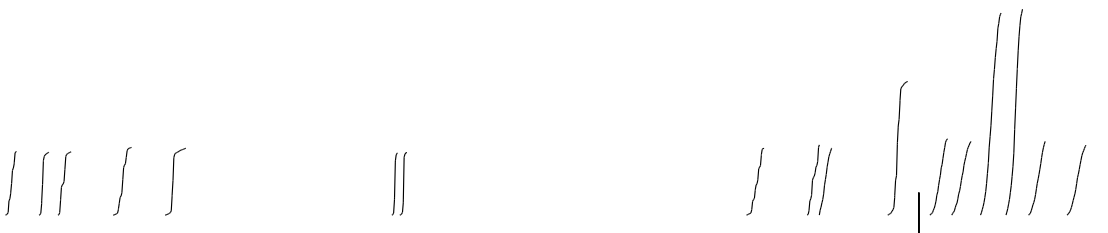

${ }^{1} \mathrm{H}$ NMR $\left(400 \mathrm{MHz}, \mathrm{CD}_{3} \mathrm{C}(\mathrm{O}) \mathrm{CD}_{3}\right)$

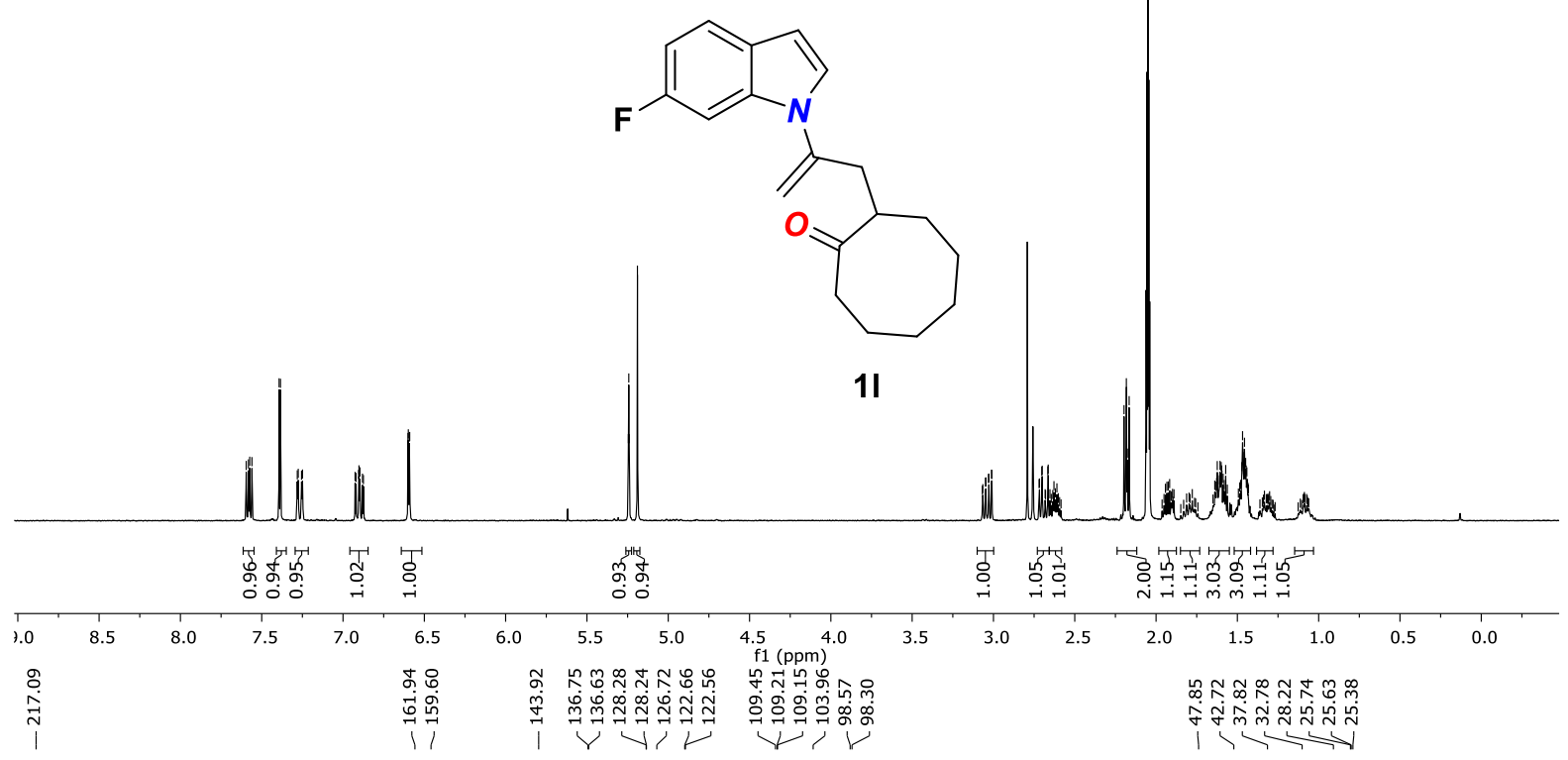

${ }^{13} \mathrm{C}$ NMR $\left(101 \mathrm{MHz}, \mathrm{CD}_{3} \mathrm{C}(\mathrm{O}) \mathrm{CD}_{3}\right)$

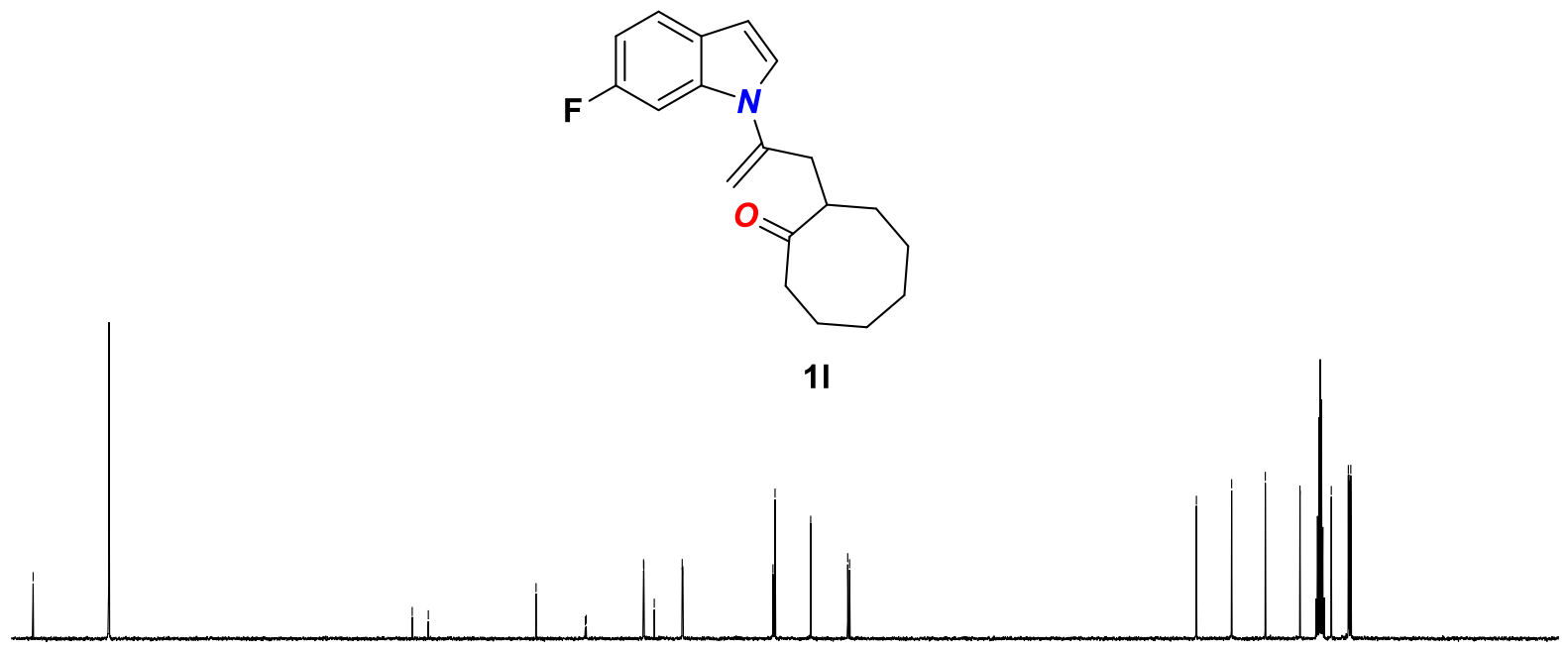

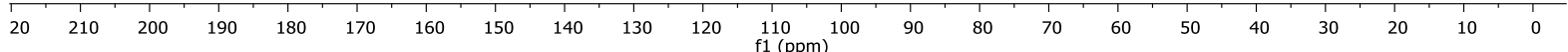


${ }^{19} \mathrm{~F} \mathrm{NMR}\left(376 \mathrm{MHz}, \mathrm{CD}_{3} \mathrm{C}(\mathrm{O}) \mathrm{CD}_{3}\right)$

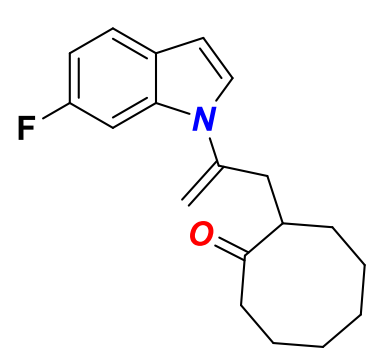

11

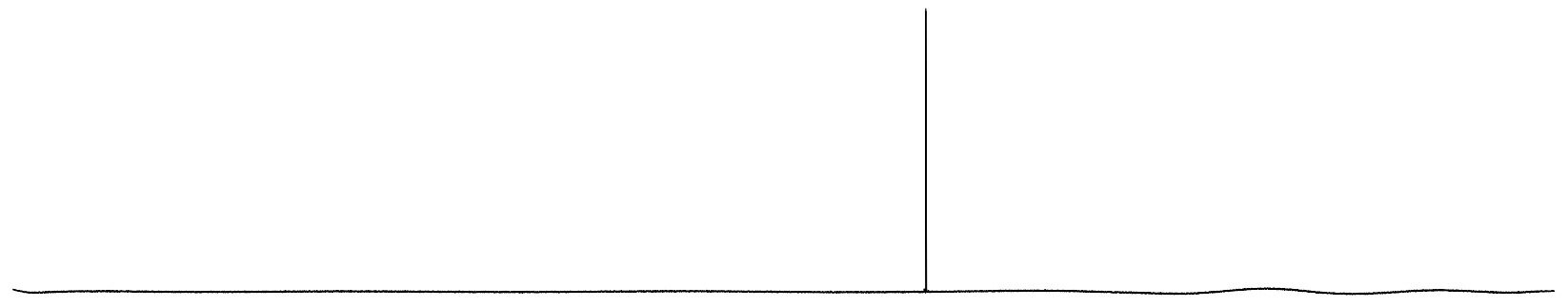

$\begin{array}{lllllllllllllllllllllllllllllll}1 & 0 & 0 & -10 & -20 & -30 & -40 & -50 & -60 & -70 & -80 & -90 & -100 & -110 & -120 & -130 & -140 & -150 & -160 & -170 & -180 & -190 & -200 & -210 & 1\end{array}$ 


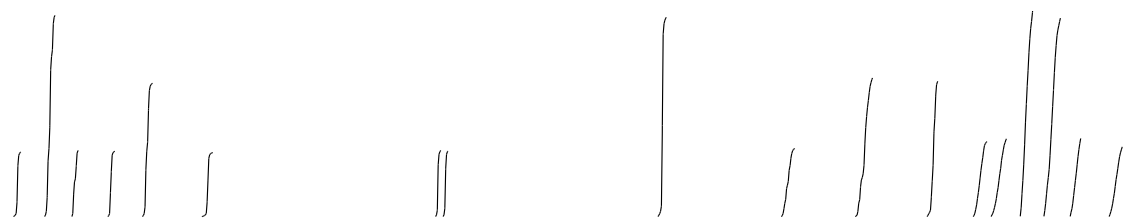

${ }^{1} \mathrm{H}$ NMR $\left(400 \mathrm{MHz}, \mathrm{CD}_{2} \mathrm{Cl}_{2}\right)$

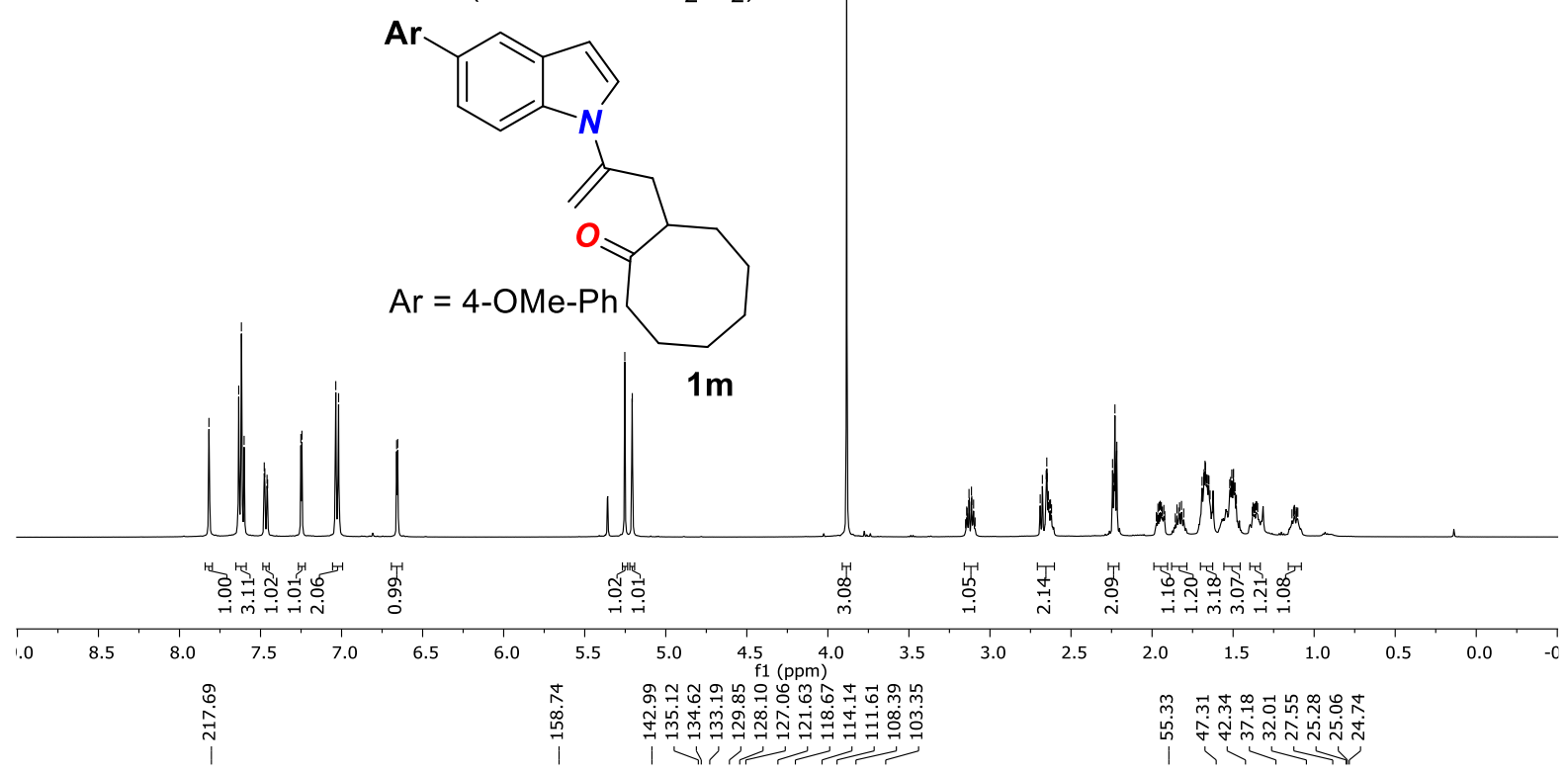

${ }^{13} \mathrm{C}$ NMR $\left(101 \mathrm{MHz}, \mathrm{CD}_{2} \mathrm{Cl}_{2}\right)$

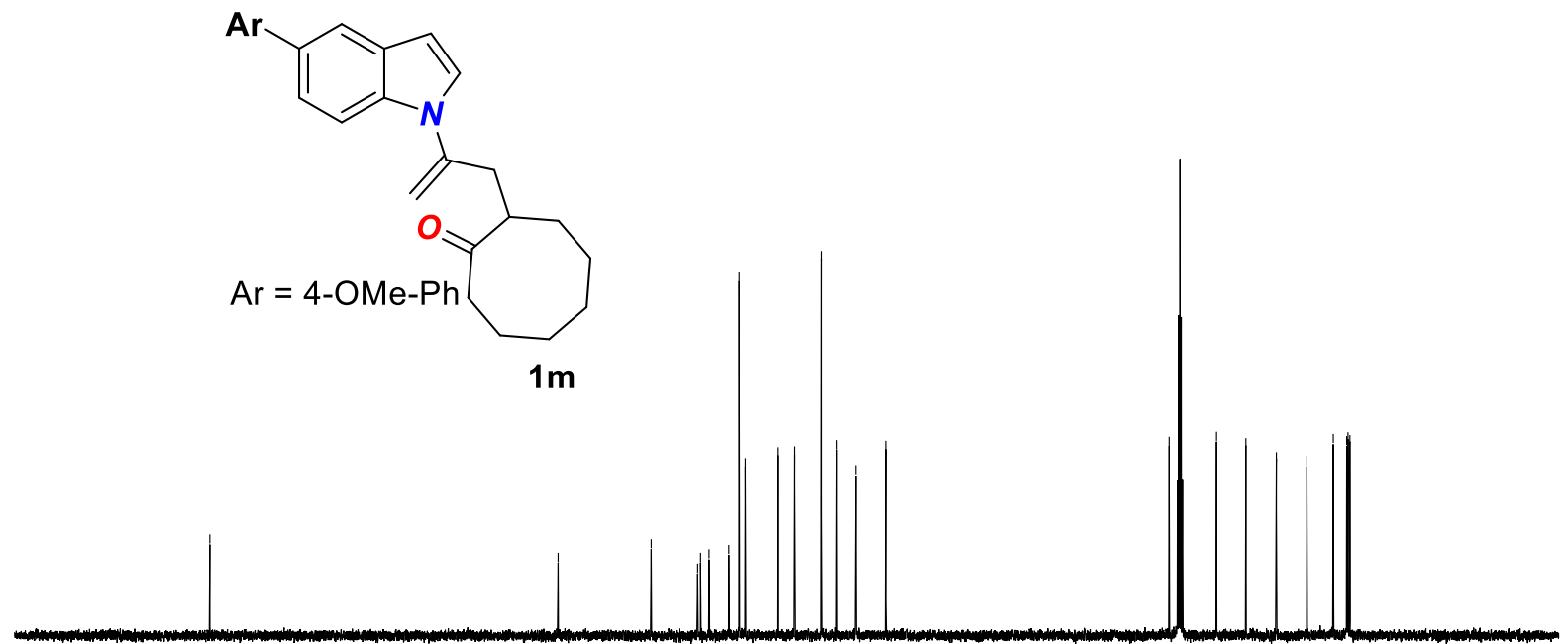

$\begin{array}{llllllllllllllllllllllllllllllllllllllllll}150 & 240 & 230 & 220 & 210 & 200 & 190 & 180 & 170 & 160 & 150 & 140 & 130 & 120 & 110 & 100 & 90 & 80 & 70 & 60 & 50 & 40 & 30 & 20 & 10 & 0 & -1\end{array}$ 


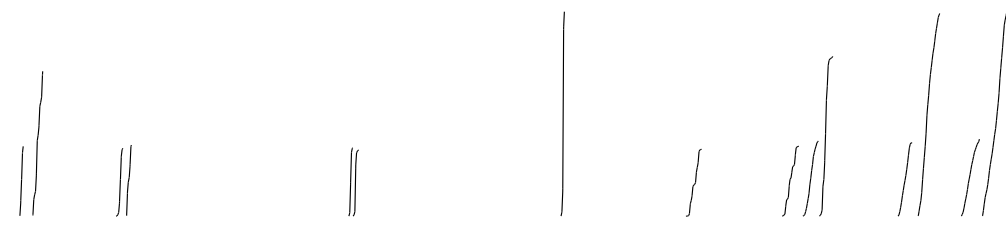

${ }^{1} \mathrm{H}$ NMR $\left(400 \mathrm{MHz}, \mathrm{CD}_{3} \mathrm{C}(\mathrm{O}) \mathrm{CD}_{3}\right)$

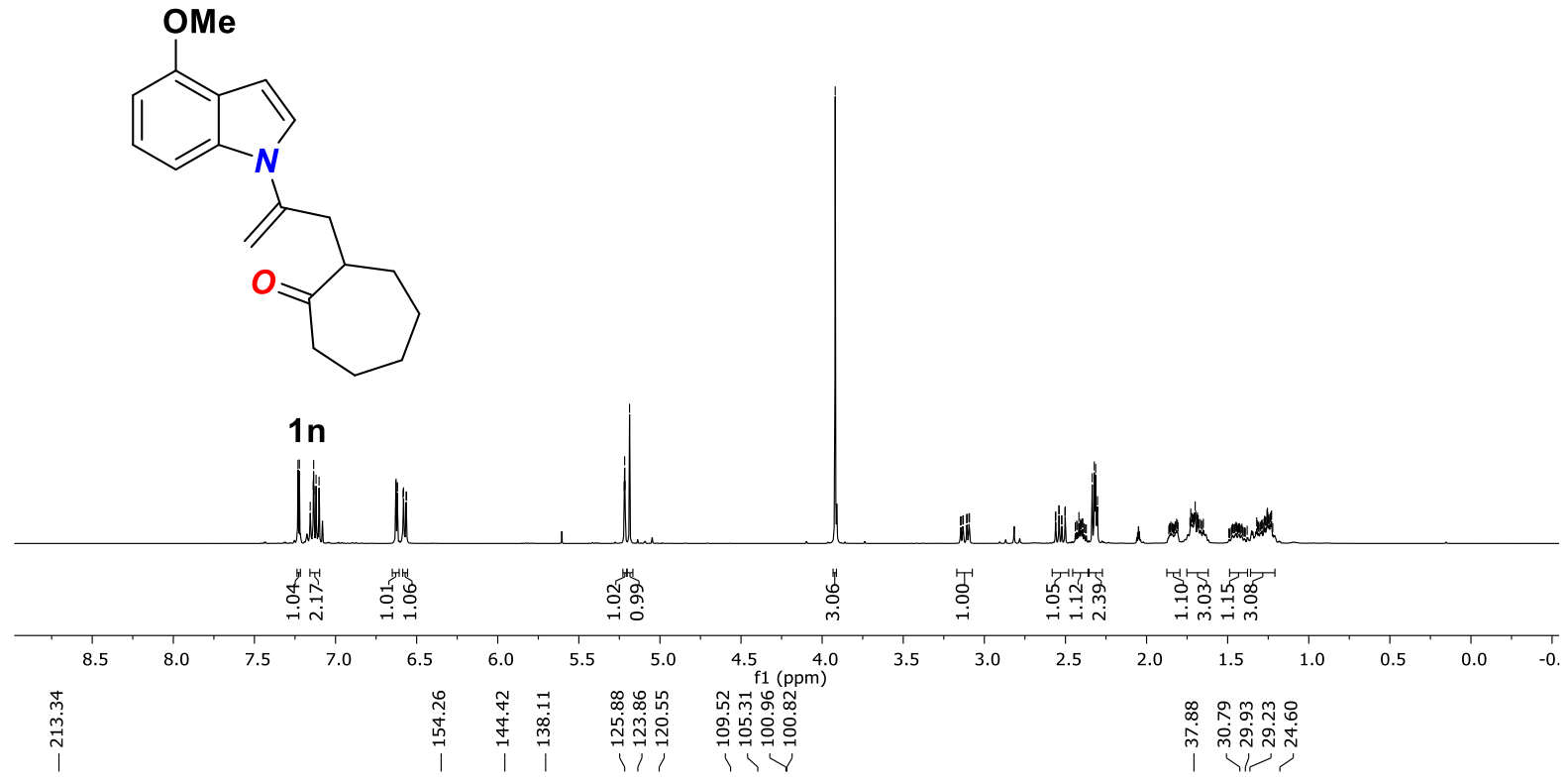

${ }^{13} \mathrm{C}$ NMR $\left(101 \mathrm{MHz}, \mathrm{CD}_{3} \mathrm{C}(\mathrm{O}) \mathrm{CD}_{3}\right)$

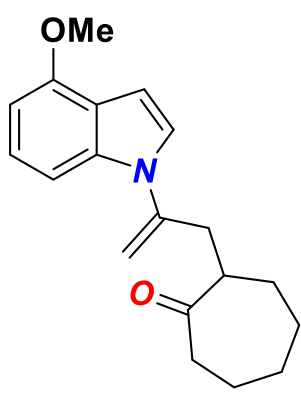

$1 \mathrm{n}$

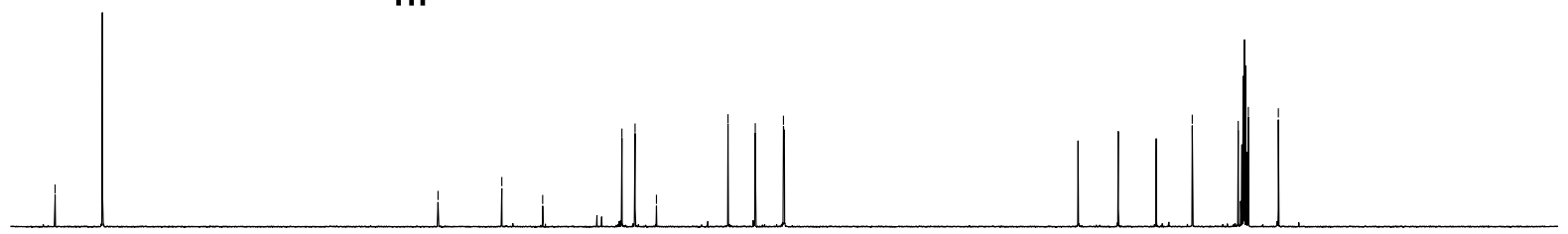

$\begin{array}{lllllllllllllllllllllllll}20 & 210 & 200 & 190 & 180 & 170 & 160 & 150 & 140 & 130 & 120 & 110 & 100 & 90 & 80 & 70 & 60 & 50 & 40 & 30 & 20 & 10 & 0 & -10\end{array}$ 


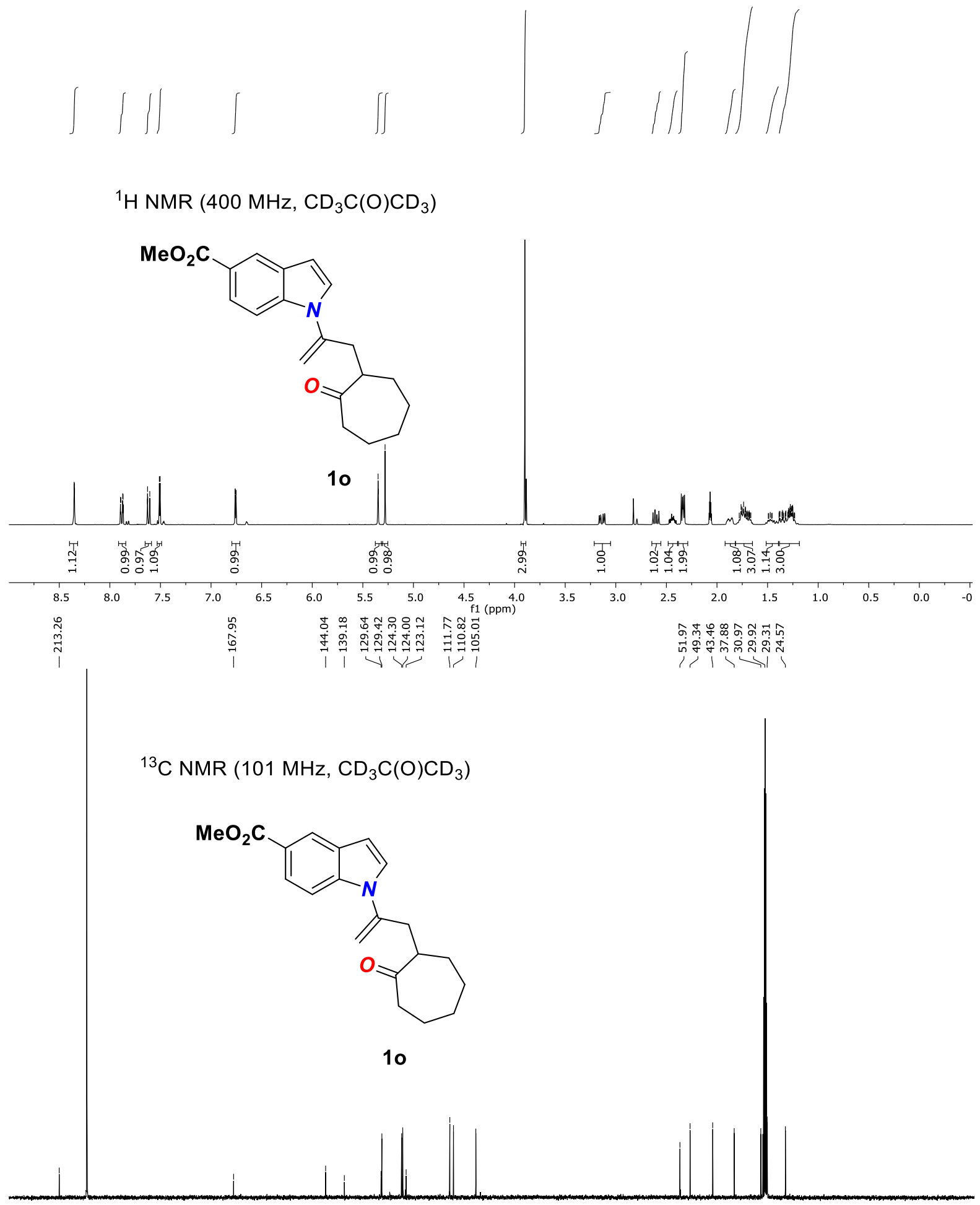

$\begin{array}{lllllllllllllllllllllllllllllllllll}220 & 210 & 200 & 190 & 180 & 170 & 160 & 150 & 140 & 130 & 120 & 110 & 100 & 90 & 80 & 70 & 60 & 50 & 40 & 30 & 20 & 10 & 0 & -10 & -20\end{array}$ 


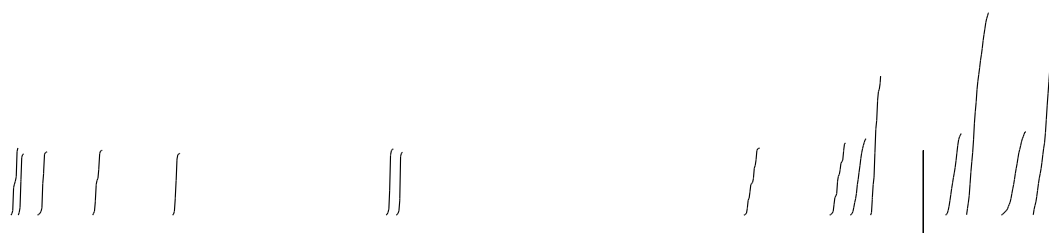

${ }^{1} \mathrm{H}$ NMR $\left(400 \mathrm{MHz}, \mathrm{CD}_{3} \mathrm{C}(\mathrm{O}) \mathrm{CD}_{3}\right)$

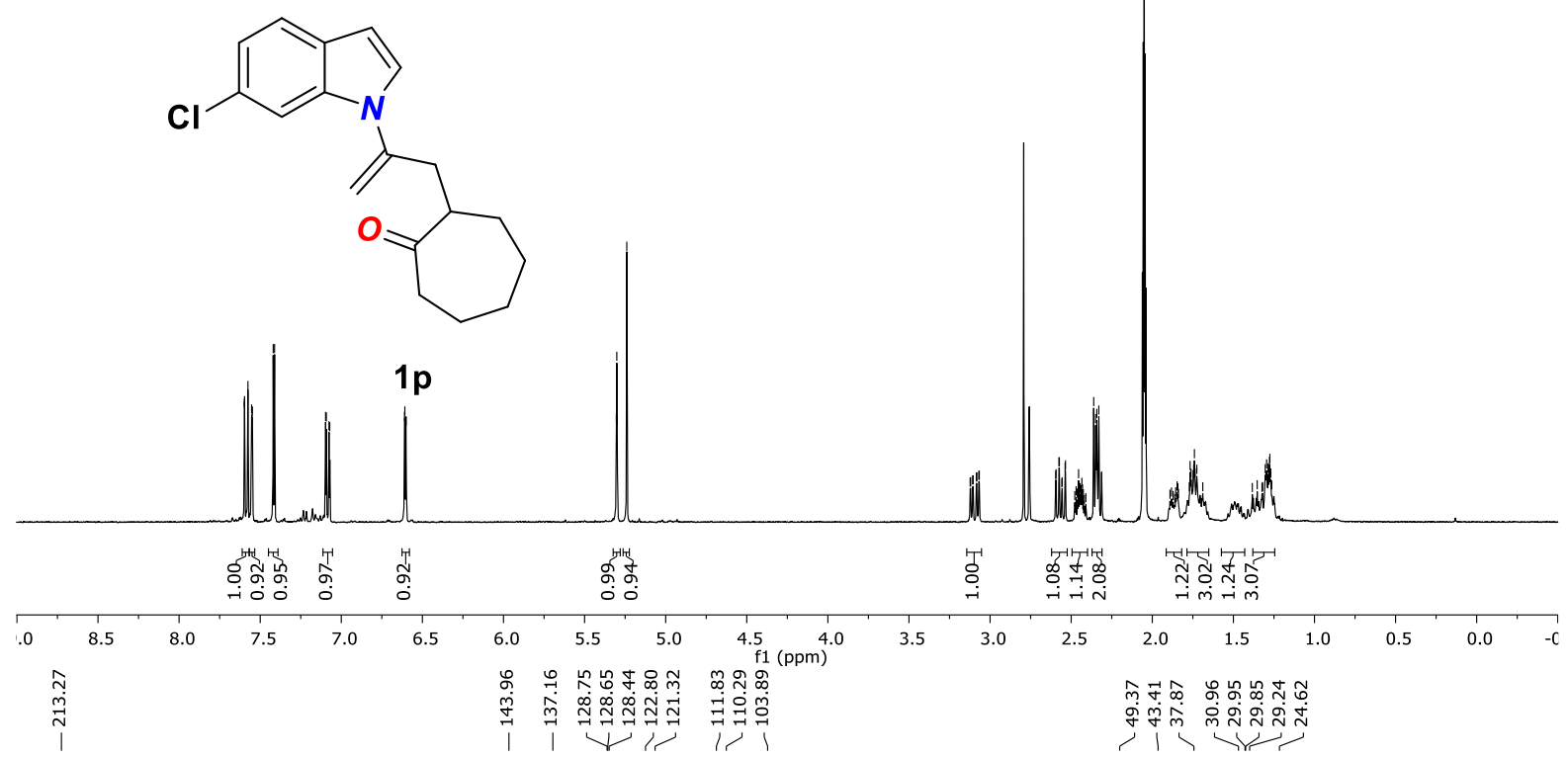

${ }^{13} \mathrm{C}$ NMR $\left(101 \mathrm{MHz}, \mathrm{CD}_{3} \mathrm{C}(\mathrm{O}) \mathrm{CD}_{3}\right)$

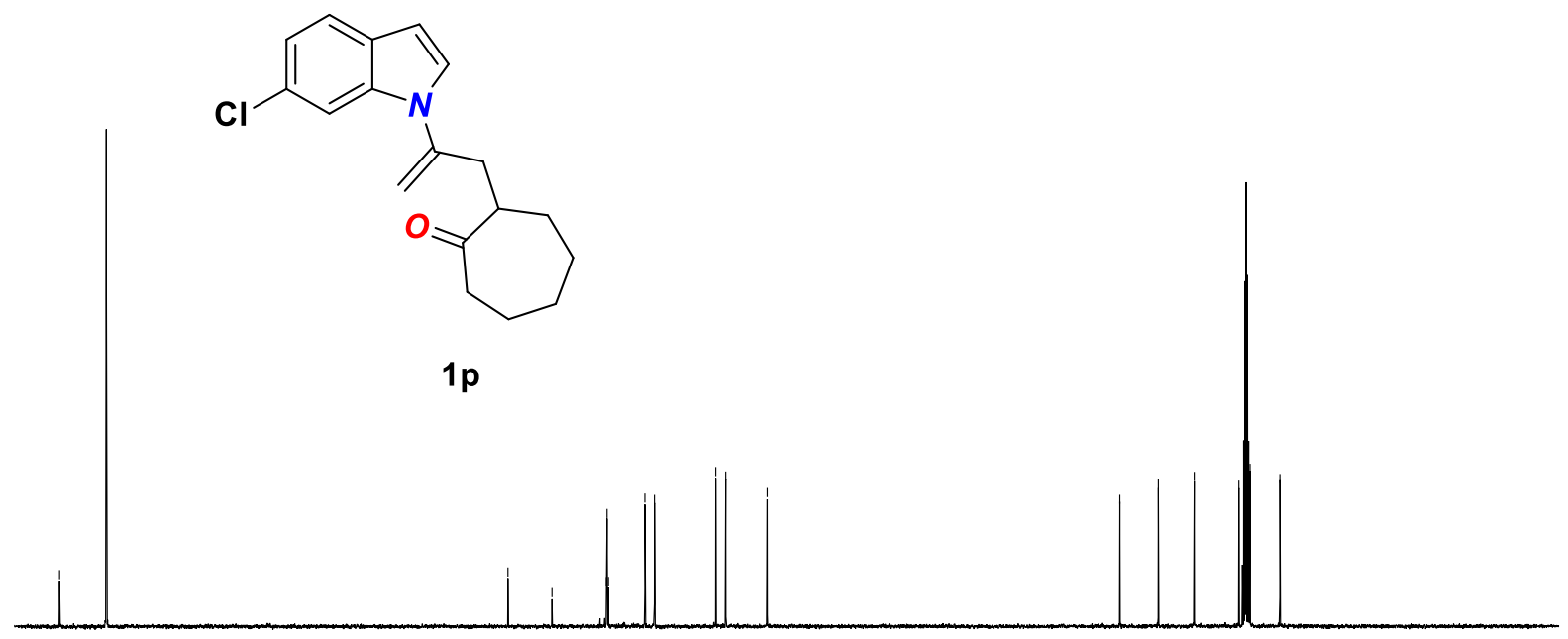

\begin{tabular}{llllllllllllllllllllllllll}
\hline 20 & 210 & 200 & 190 & 180 & 170 & 160 & 150 & 140 & 130 & 120 & 110 & 100 & 90 & 80 & 70 & 60 & 50 & 40 & 30 & 20 & 10 & 0 & -10
\end{tabular} 


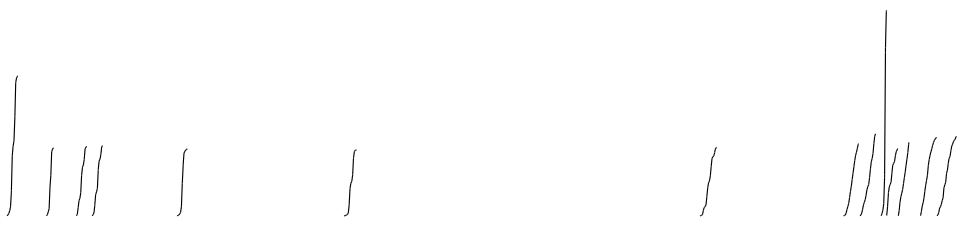

${ }^{1} \mathrm{H}$ NMR $\left(400 \mathrm{MHz}, \mathrm{CD}_{3} \mathrm{C}(\mathrm{O}) \mathrm{CD}_{3}\right)$
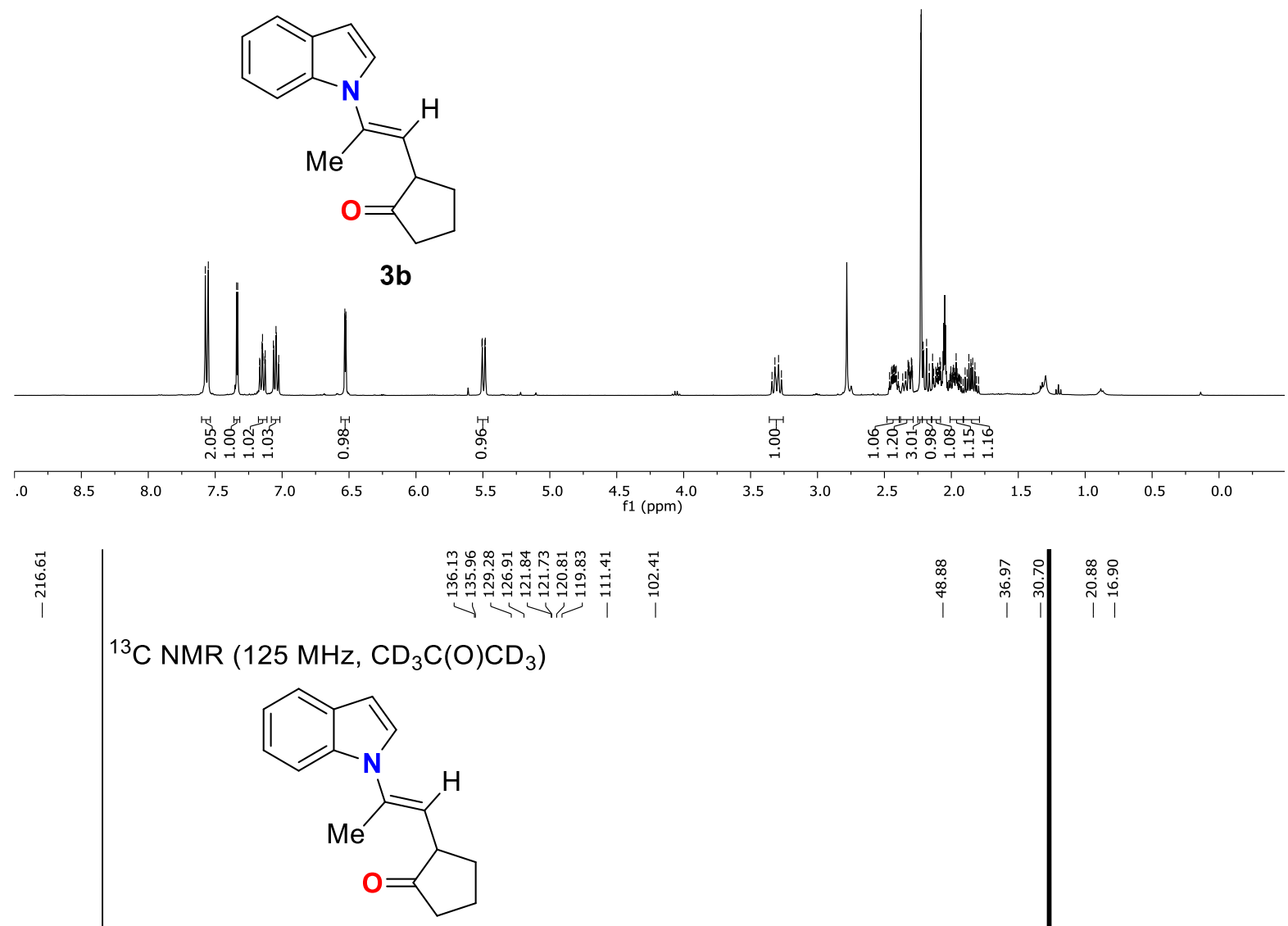

3b

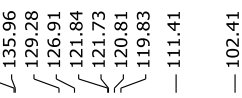

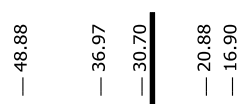

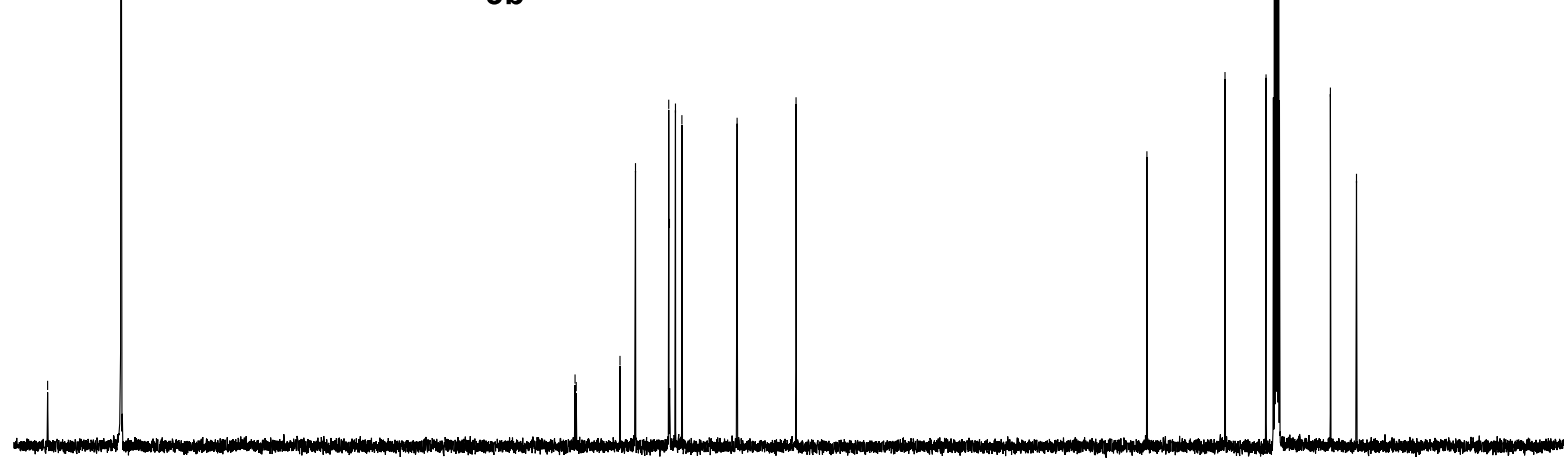

$\begin{array}{llllllllllllllllllllllllllllllllll}220 & 210 & 200 & 190 & 180 & 170 & 160 & 150 & 140 & 130 & 120 & 110 & 100 & 90 & 80 & 70 & 60 & 50 & 40 & 30 & 20 & 10 & 0 & -10\end{array}$ SI-56 

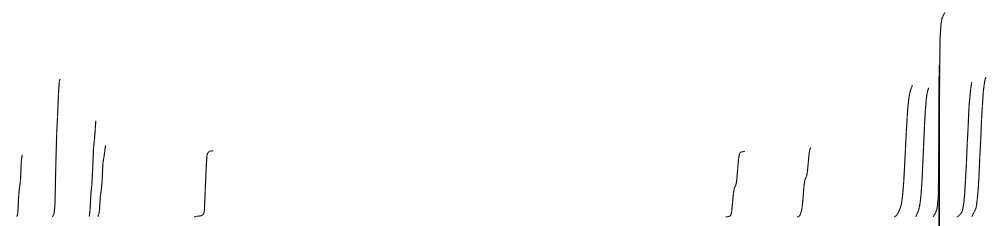

${ }^{1} \mathrm{H}$ NMR (500 MHz, $\left.\mathrm{CDCl}_{3}\right)$

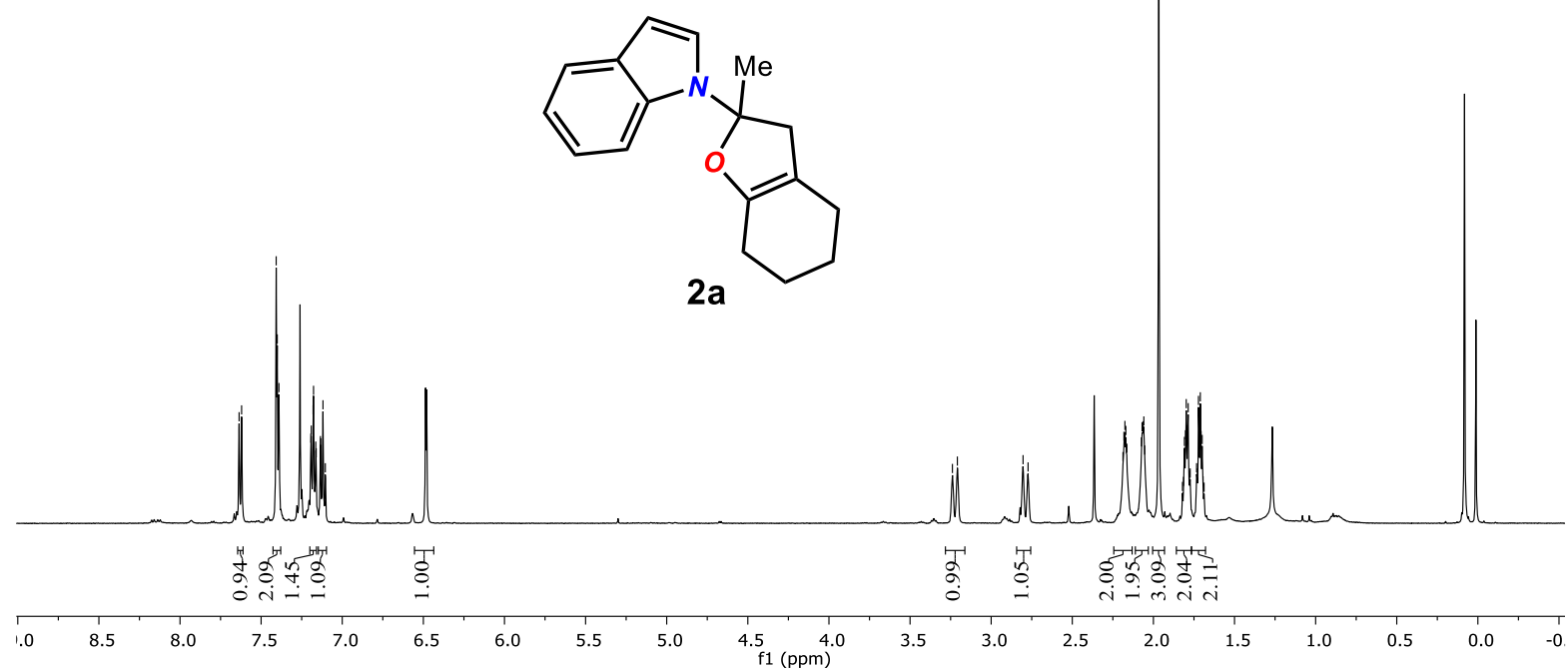

${ }^{13} \mathrm{C}$ NMR $\left(125 \mathrm{MHz}, \mathrm{CDCl}_{3}\right)$<smiles>CC1(n2ccc3ccccc32)CC2=C(CCCC2)O1</smiles>

2a

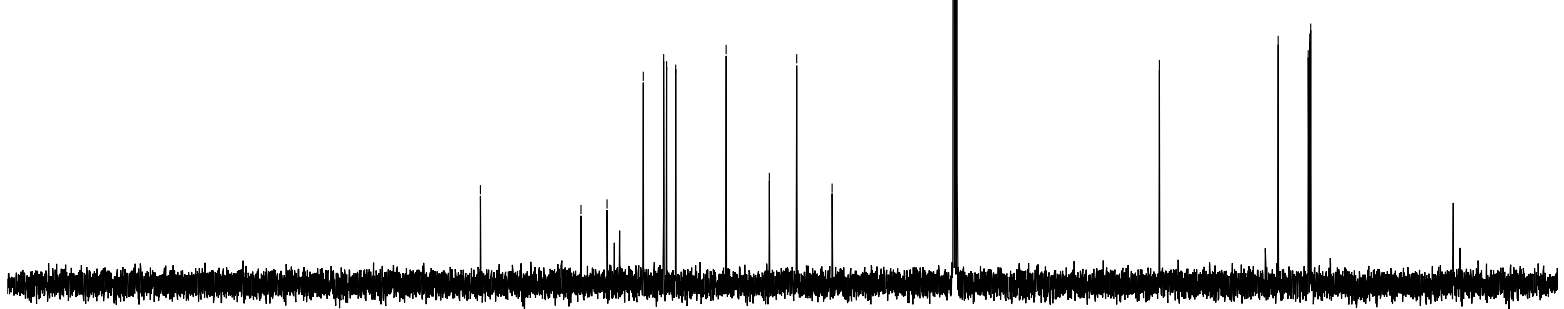

$\begin{array}{llllllllllll}220 & 210 & 200 & 190 & 180 & 170 & 160 & 150 & 140 & 130 & 120 & \begin{array}{l}110 \\ \mathrm{f} 1(\mathrm{ppm})\end{array}\end{array}$ 


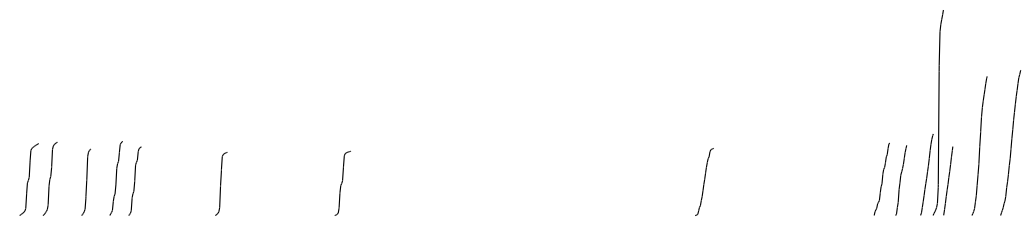

${ }^{1} \mathrm{H}$ NMR $\left(400 \mathrm{MHz}, \mathrm{CD}_{3} \mathrm{C}(\mathrm{O}) \mathrm{CD}_{3}\right)$
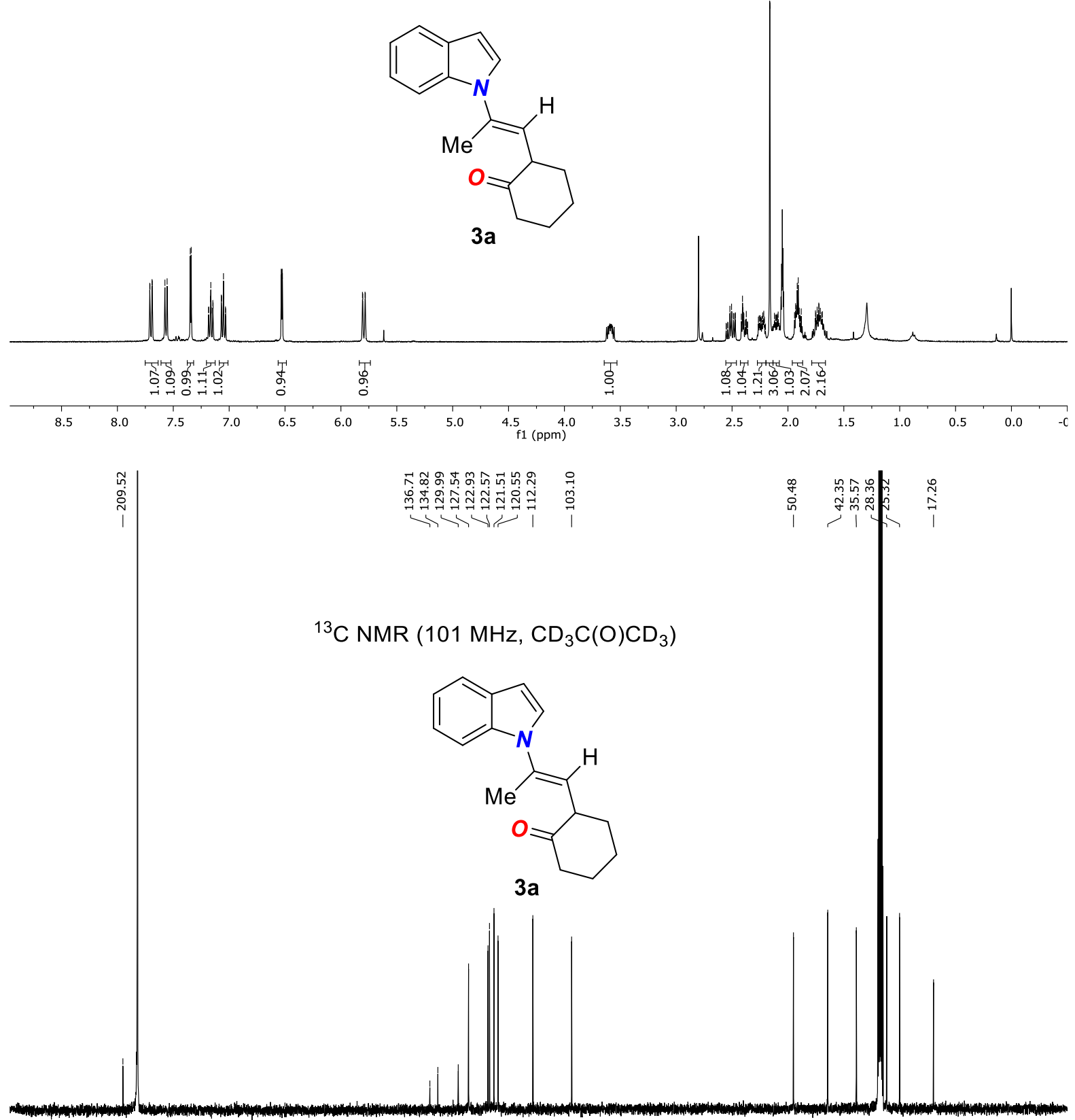

$\begin{array}{lllllllllllllllllllllllllll}230 & 220 & 210 & 200 & 190 & 180 & 170 & 160 & 150 & 140 & 130 & 120 & 110 & 100 & 90 & 80 & 70 & 60 & 50 & 40 & 30 & 20 & 10 & 0 & -10\end{array}$ 


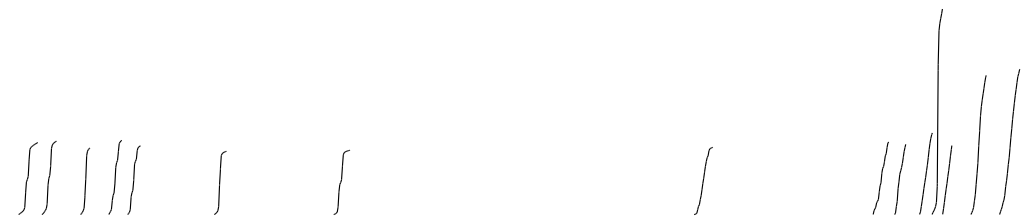

${ }^{1} \mathrm{H}$ NMR $\left(400 \mathrm{MHz}, \mathrm{CD}_{3} \mathrm{C}(\mathrm{O}) \mathrm{CD}_{3}\right)$

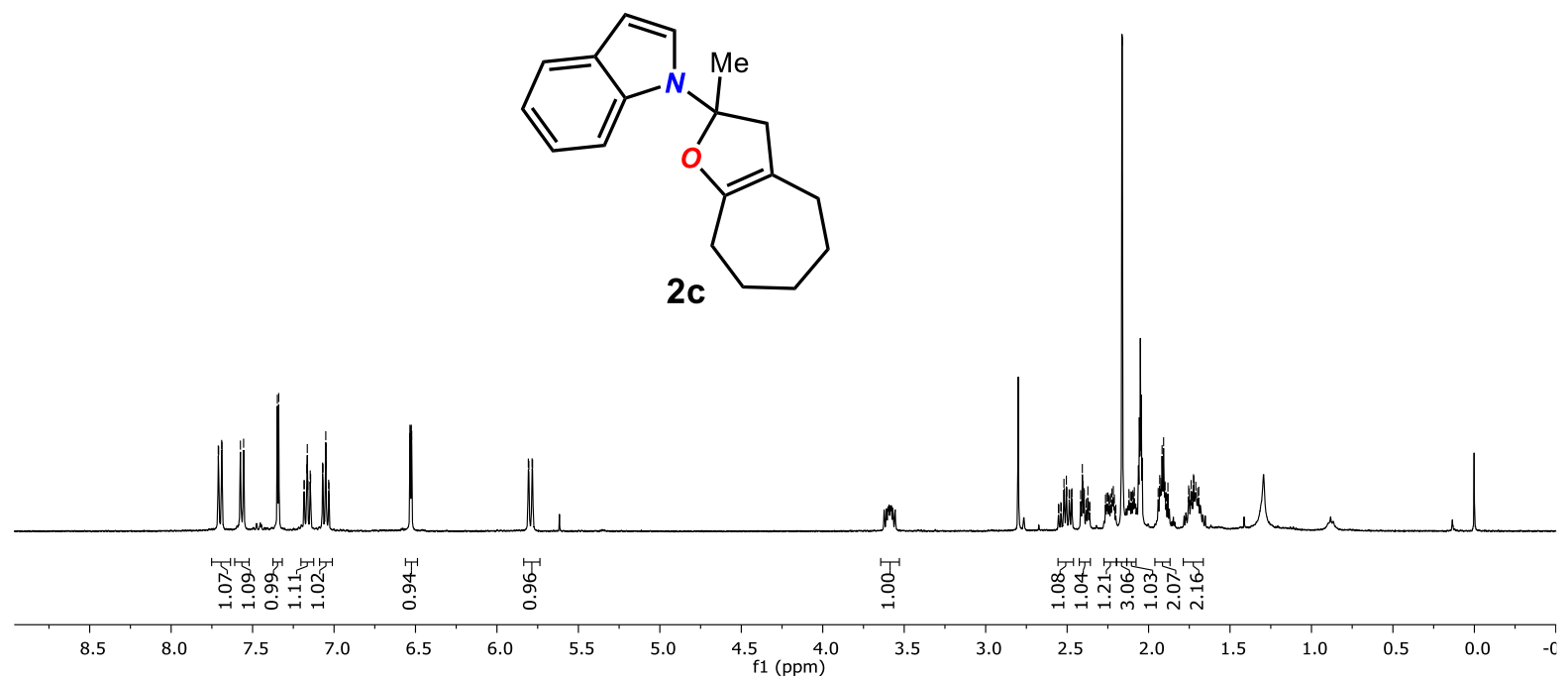

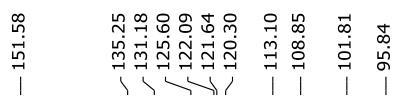

${ }^{13} \mathrm{C}$ NMR $\left(125 \mathrm{MHz}, \mathrm{CD}_{3} \mathrm{C}(\mathrm{O}) \mathrm{CD}_{3}\right)$

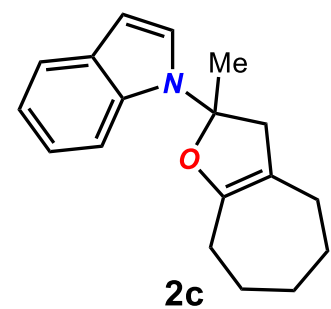

$\begin{array}{llllllllllll}220 & 210 & 200 & 190 & 180 & 170 & 160 & 150 & 140 & 130 & 120 & \begin{array}{l}110 \\ \mathrm{f} 1(\mathrm{ppm})\end{array}\end{array}$ 

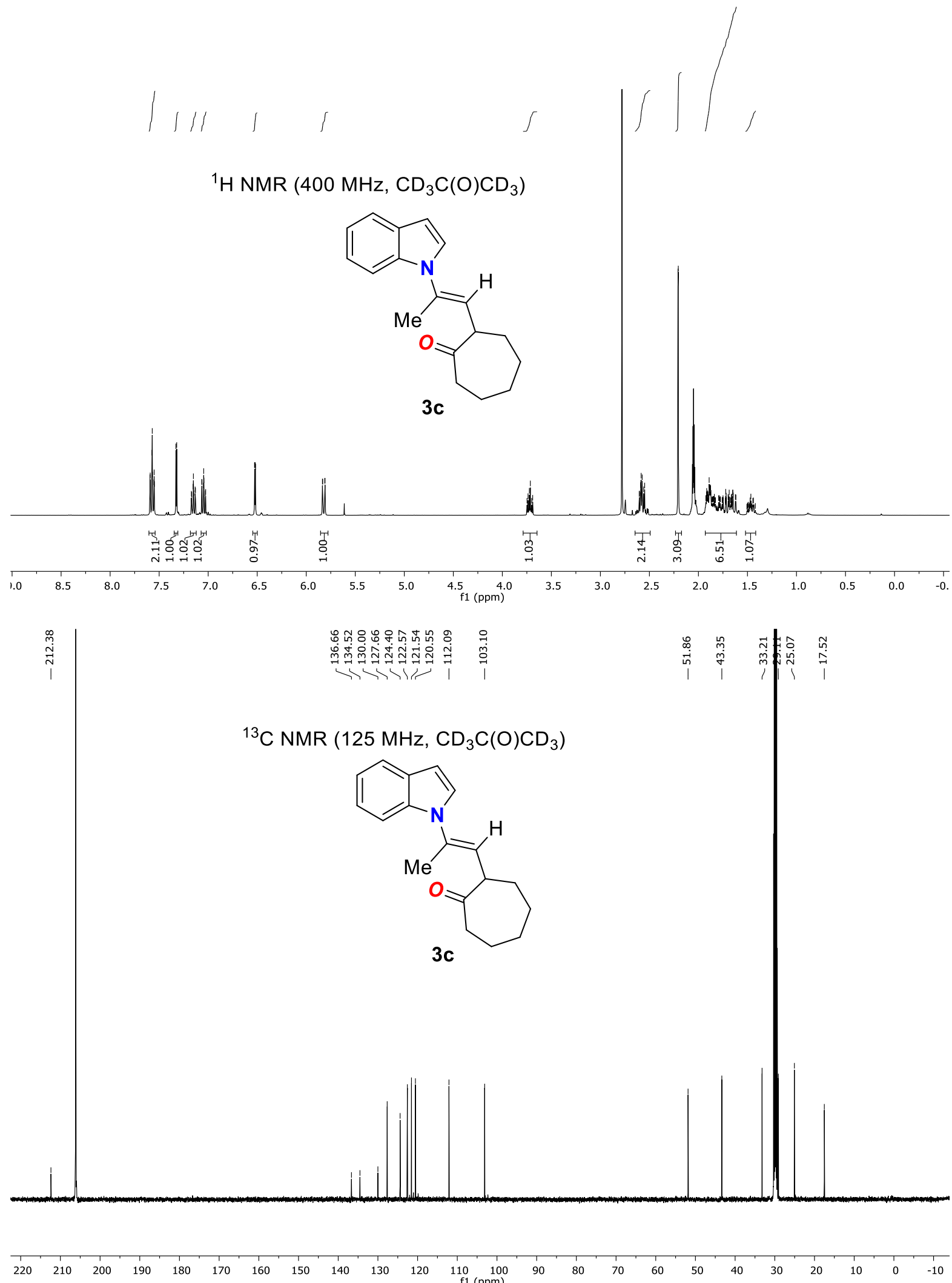

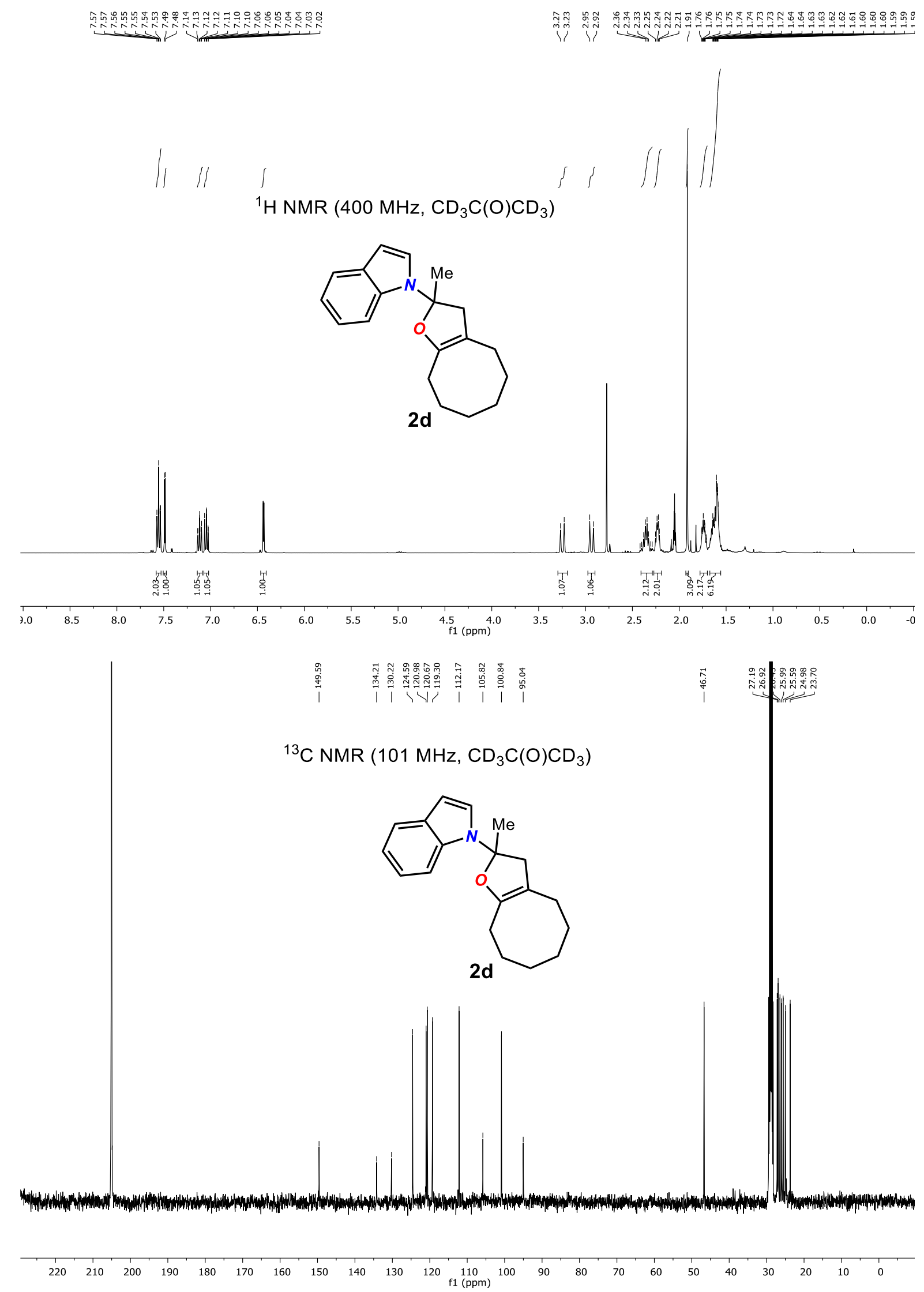

SI-61 


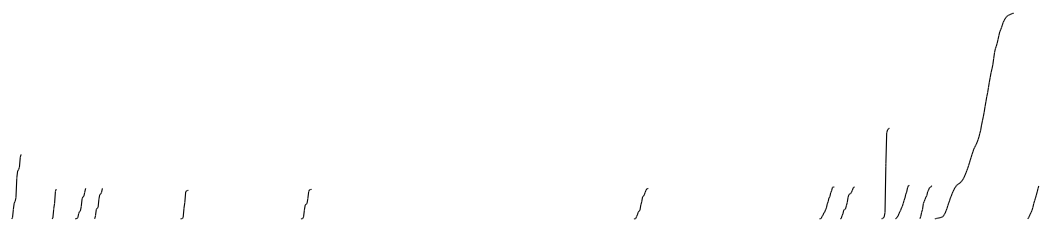

${ }^{1} \mathrm{H}$ NMR $\left(400 \mathrm{MHz}, \mathrm{CD}_{3} \mathrm{C}(\mathrm{O}) \mathrm{CD}_{3}\right)$
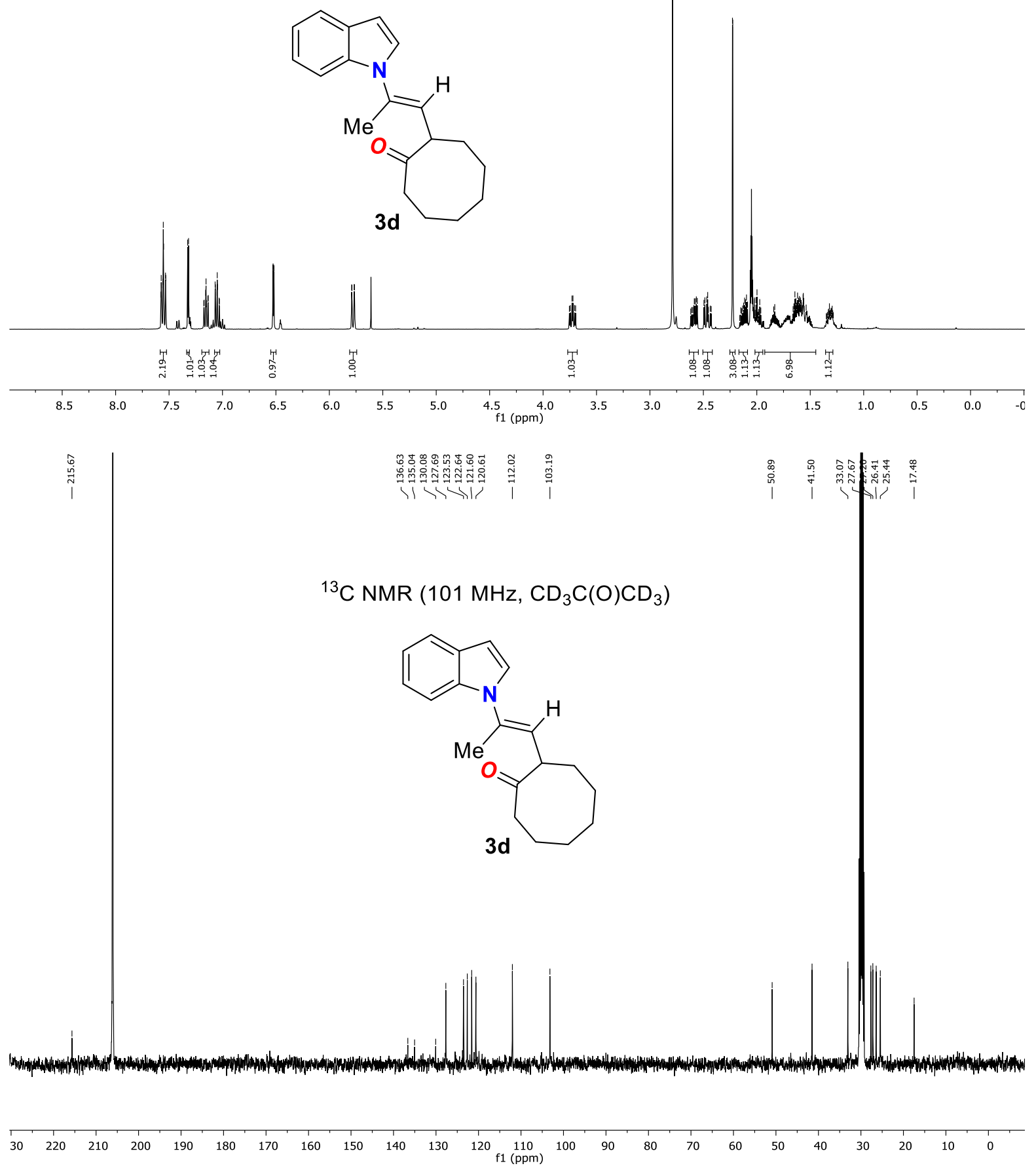

SI-62 


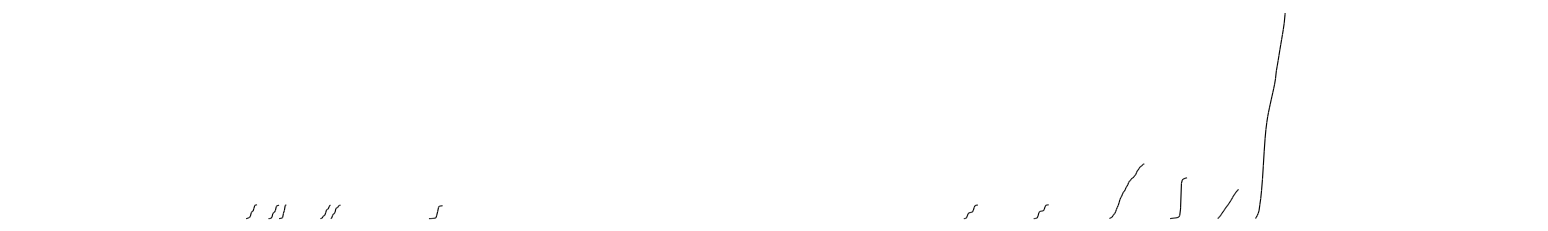

${ }^{1} \mathrm{H}$ NMR $\left(400 \mathrm{MHz}, \mathrm{CD}_{2} \mathrm{Cl}_{2}\right)$
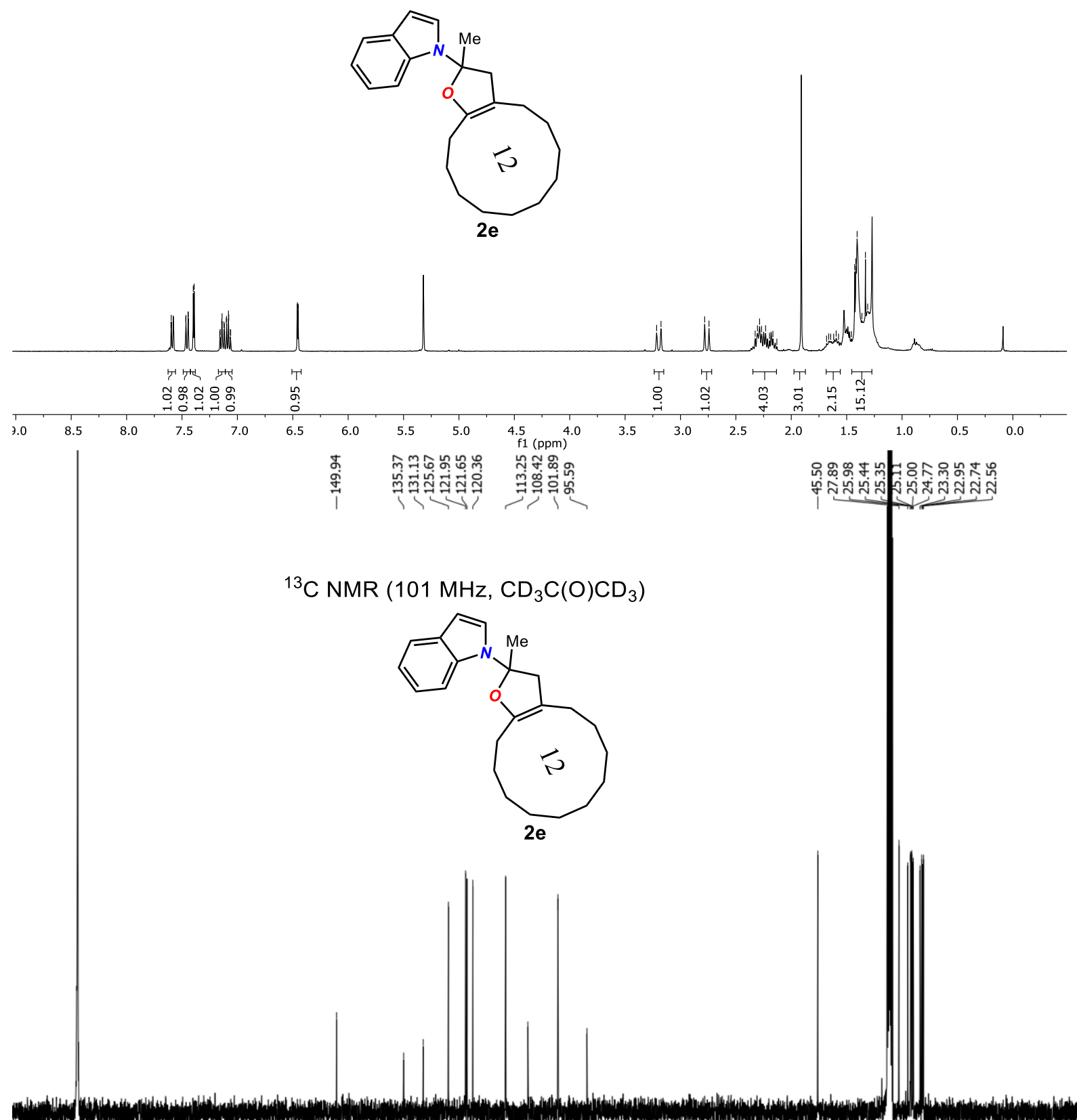

$\begin{array}{llllllllllllllllllllllll}20 & 210 & 200 & 190 & 180 & 170 & 160 & 150 & 140 & 130 & 120 & 110 & 100 & 90 & 80 & 70 & 60 & 50 & 40 & 30 & 20 & 10 & 0 & -10\end{array}$ SI-63 


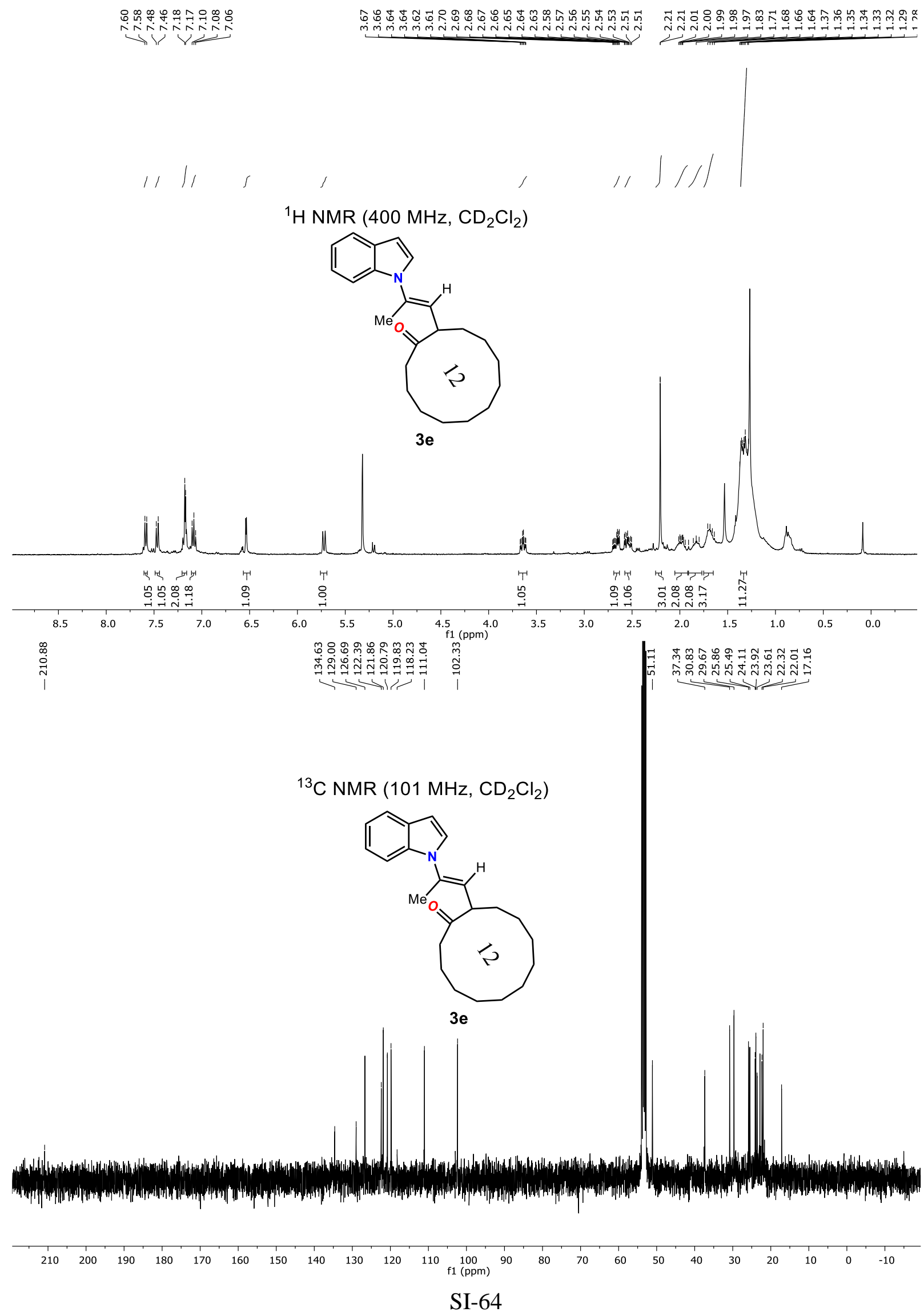




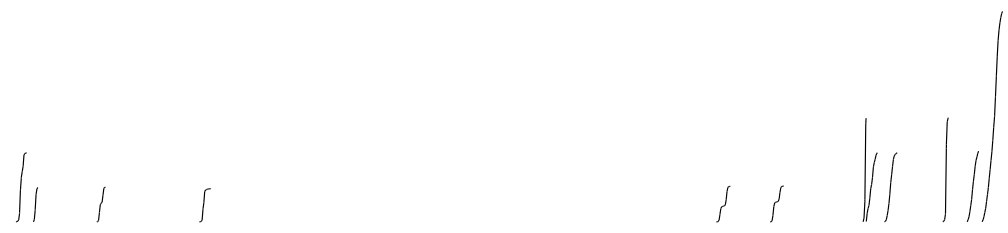

${ }^{1} \mathrm{H}$ NMR $\left(400 \mathrm{MHz}, \mathrm{CD}_{3} \mathrm{C}(\mathrm{O}) \mathrm{CD}_{3}\right)$<smiles>Cc1ccc2c(ccn2C)c1</smiles>

2f

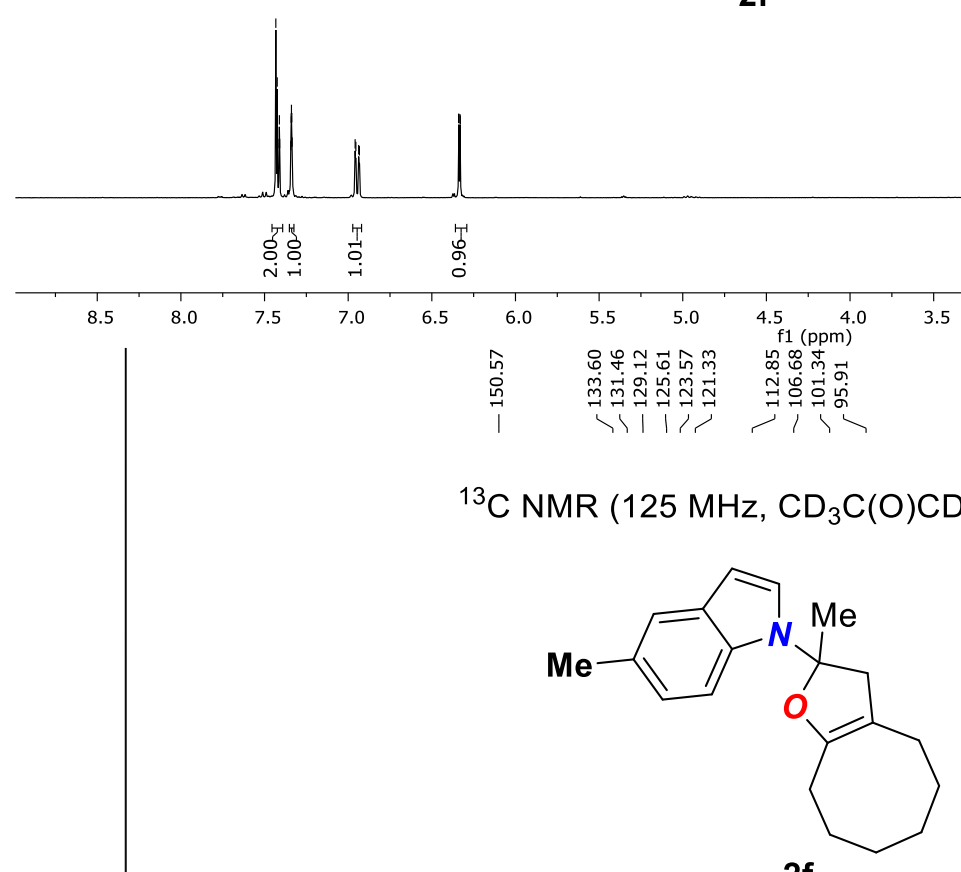

$2 f$

要

। $\quad \underbrace{\infty}$

蕉

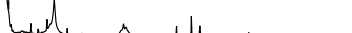

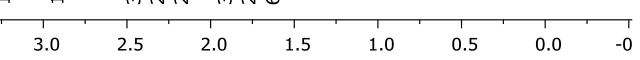

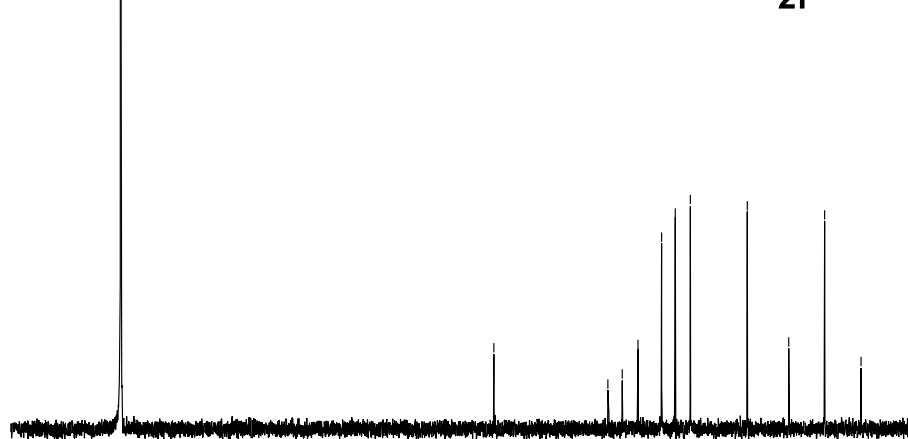

$\begin{array}{lllllllllllll}220 & 210 & 200 & 190 & 180 & 170 & 160 & 150 & 140 & 130 & 120 & 110 & 100\end{array}$

SI-65 

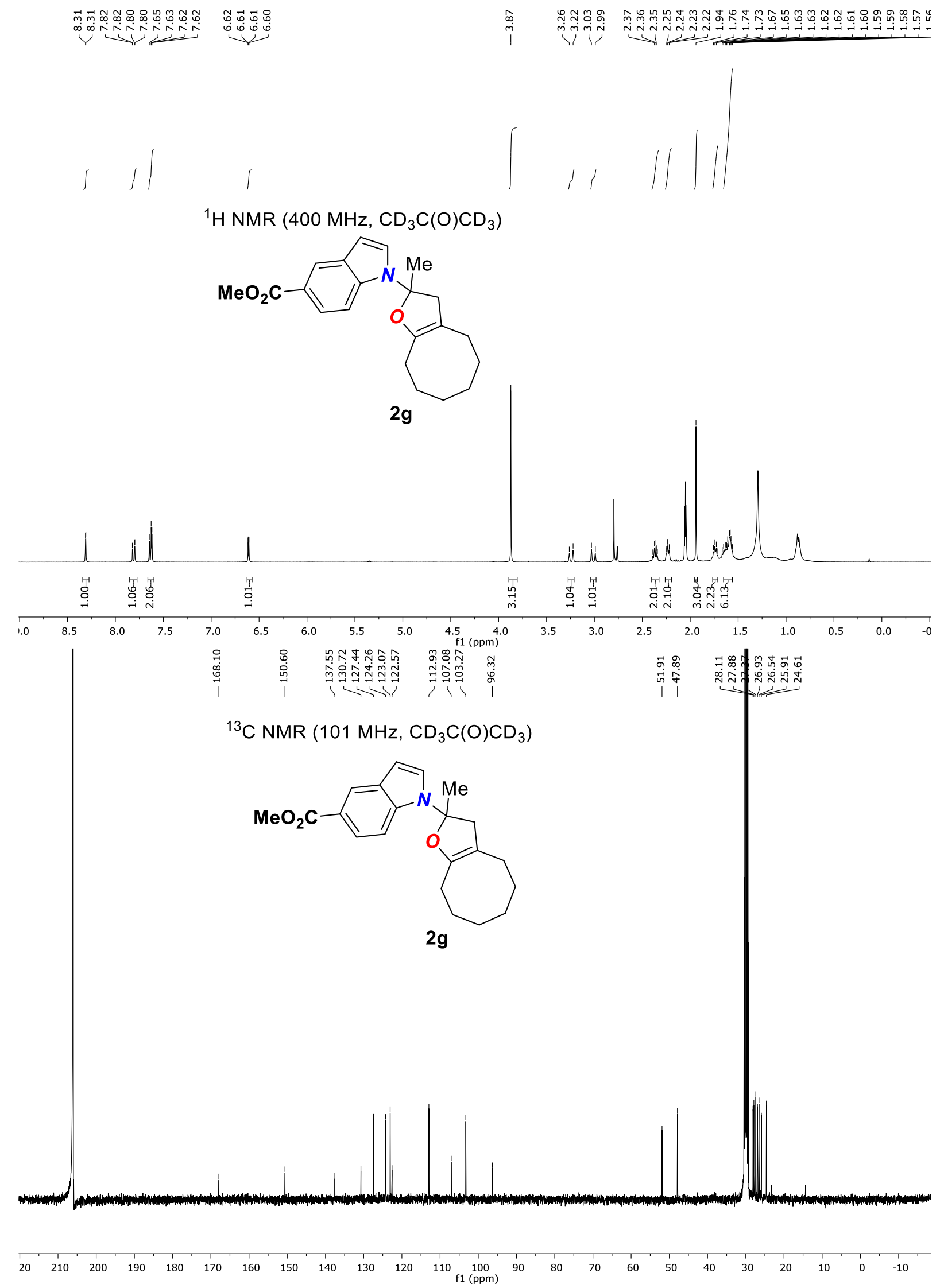


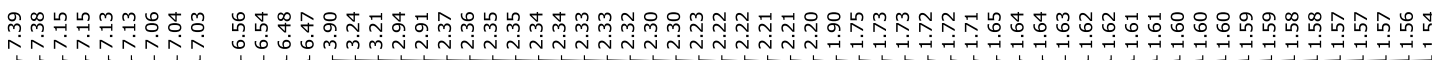

I 1111

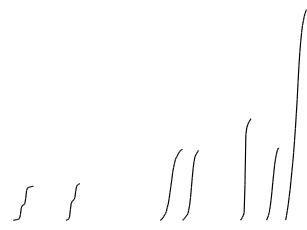

${ }^{1} \mathrm{H}$ NMR $\left(400 \mathrm{MHz}, \mathrm{CD}_{3} \mathrm{C}(\mathrm{O}) \mathrm{CD}_{3}\right)$

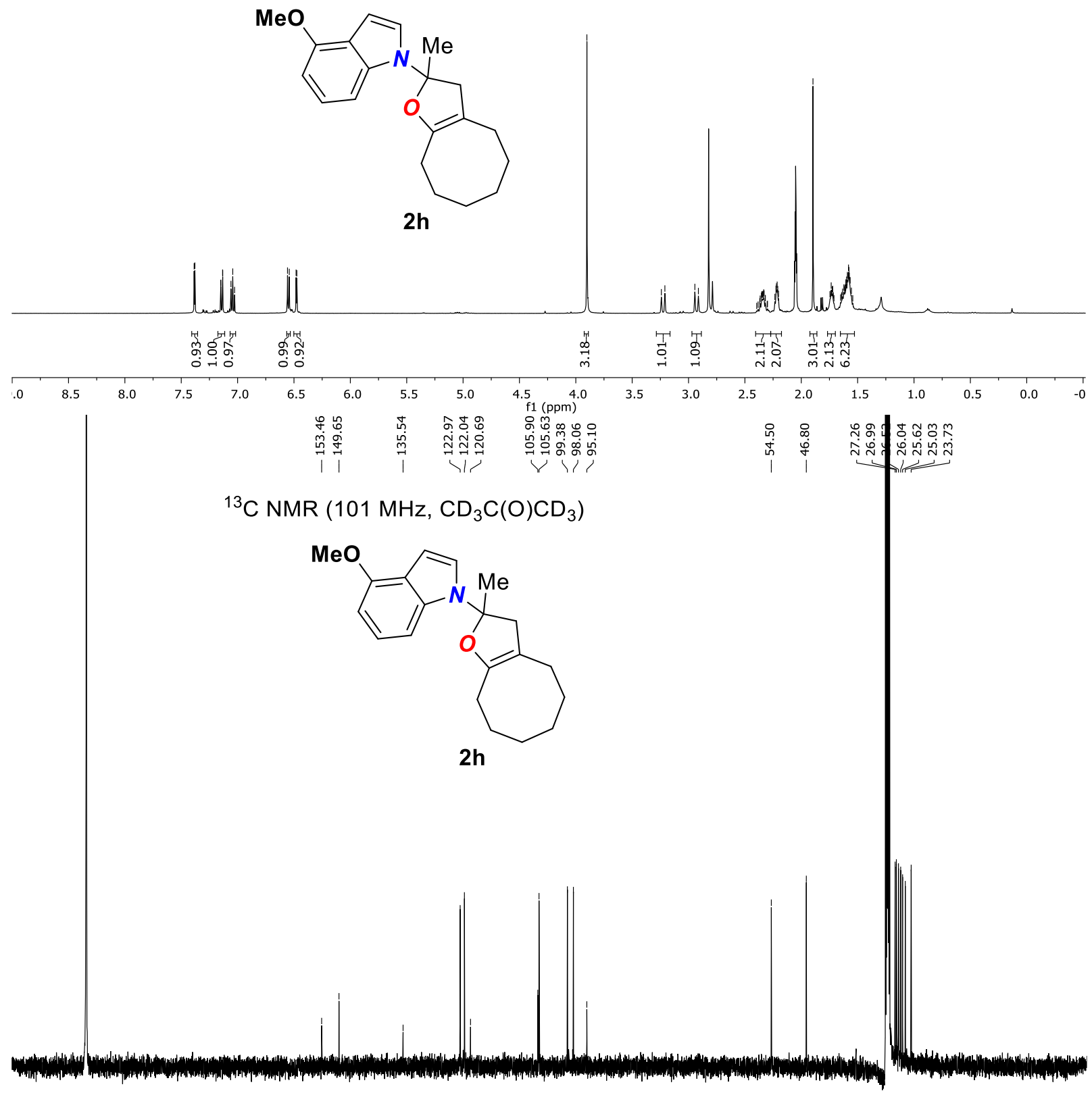

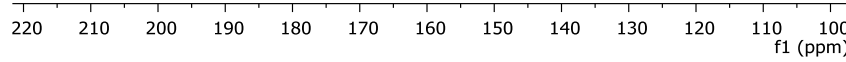
SI-67 


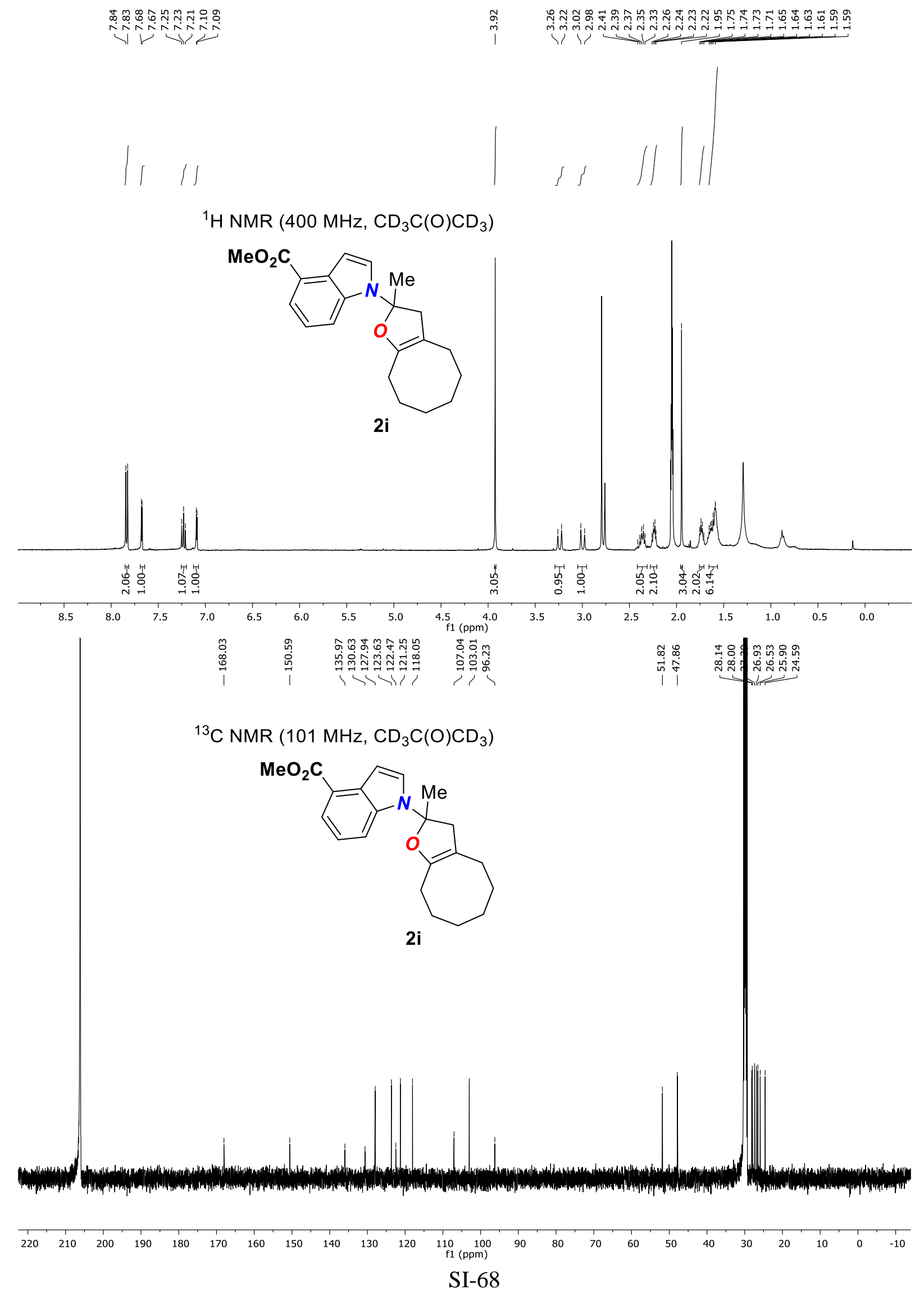




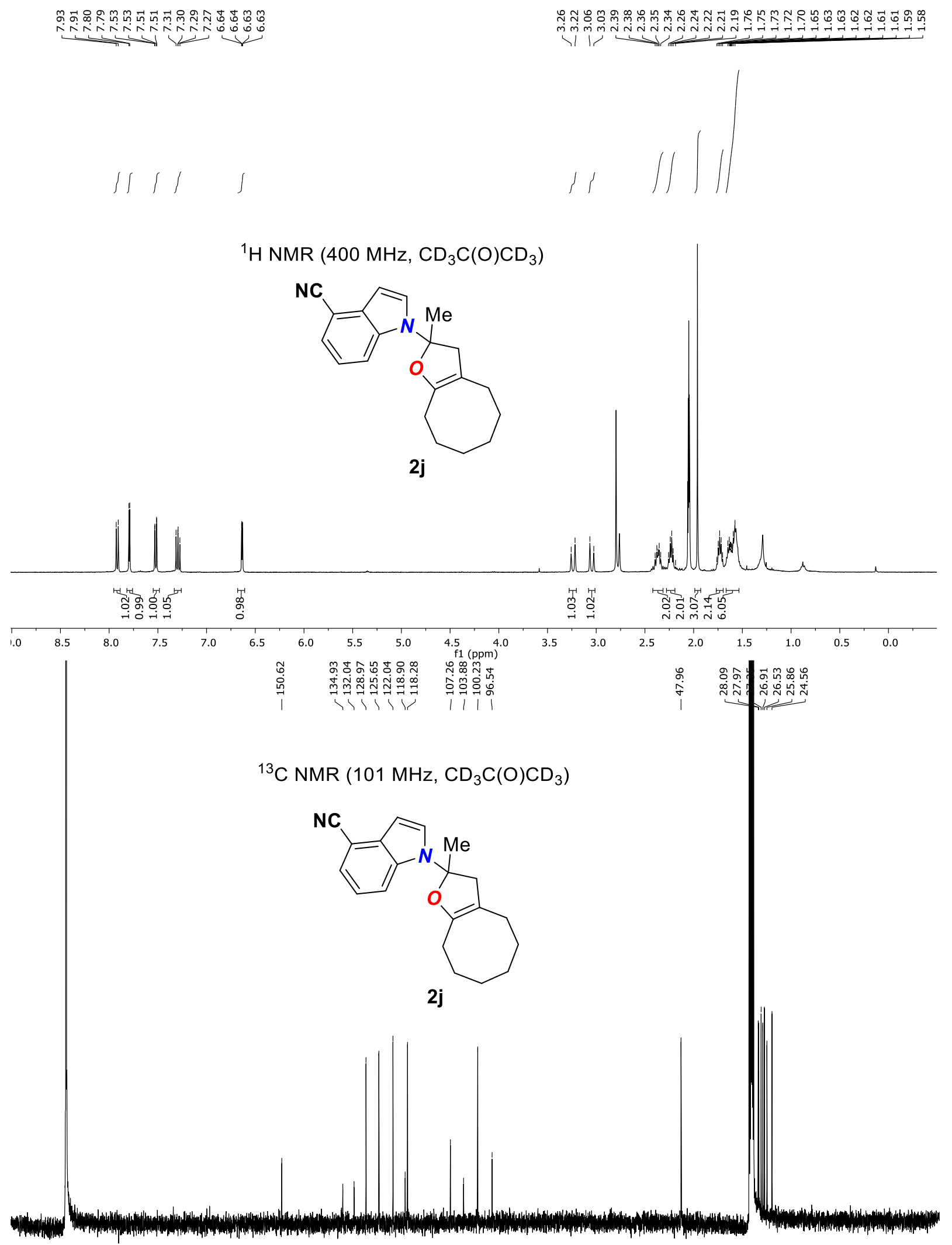

(1) 1

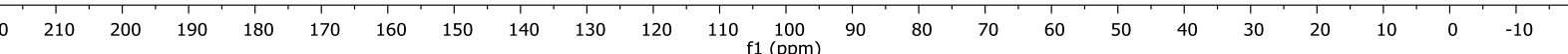
SI-69 


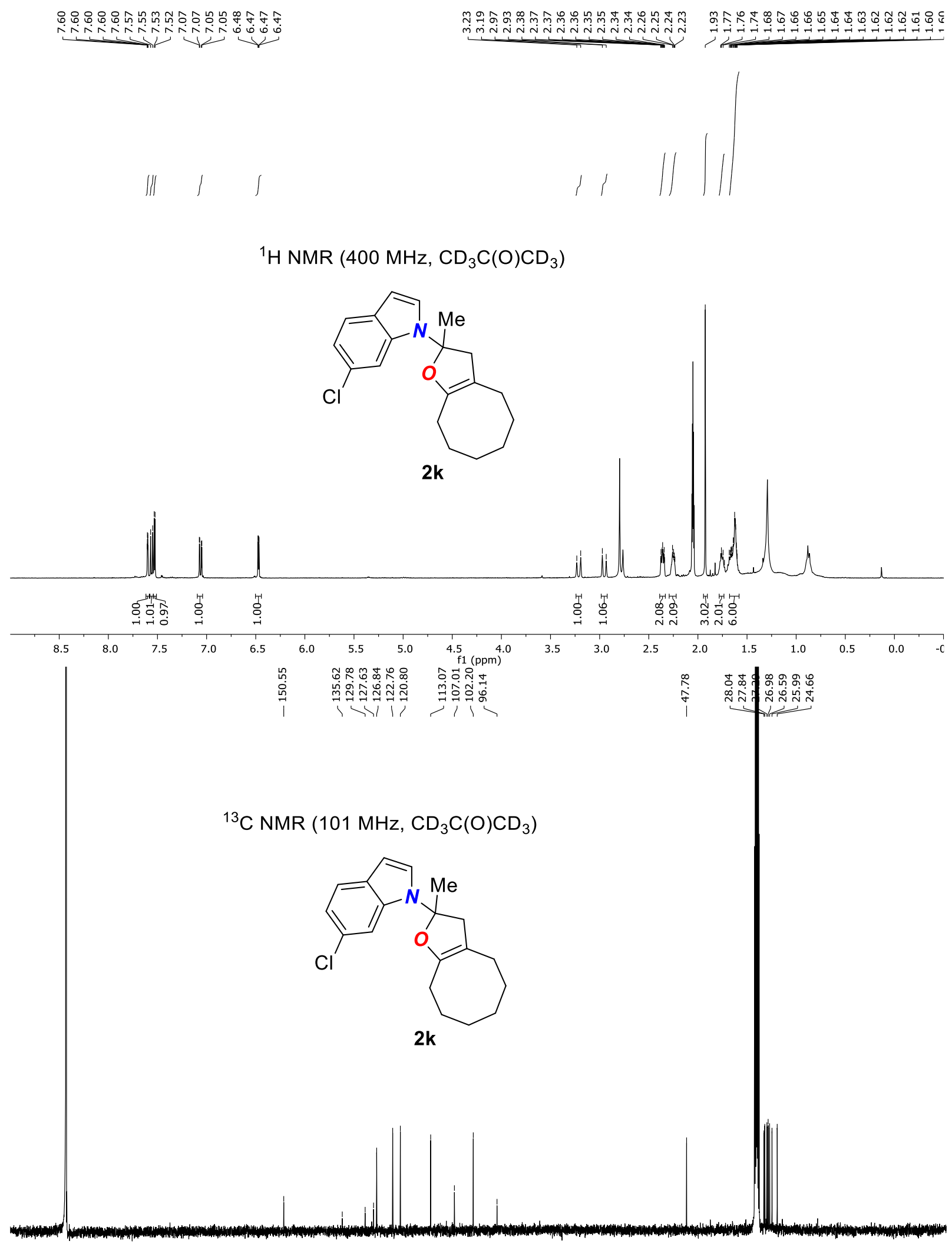

\begin{tabular}{lllllllllllllllllllllllllll}
\hline & 210 & 200 & 190 & 180 & 170 & 160 & 150 & 140 & 130 & 120 & 110 & 100 & 90 & 80 & 70 & 60 & 50 & 40 & 30 & 20 & 10 & 0 & -10
\end{tabular} SI-70 
$\iint S \int+\int\left|\int\right| \|$

${ }^{1} \mathrm{H}$ NMR $\left(400 \mathrm{MHz}, \mathrm{CD}_{3} \mathrm{C}(\mathrm{O}) \mathrm{CD}_{3}\right)$

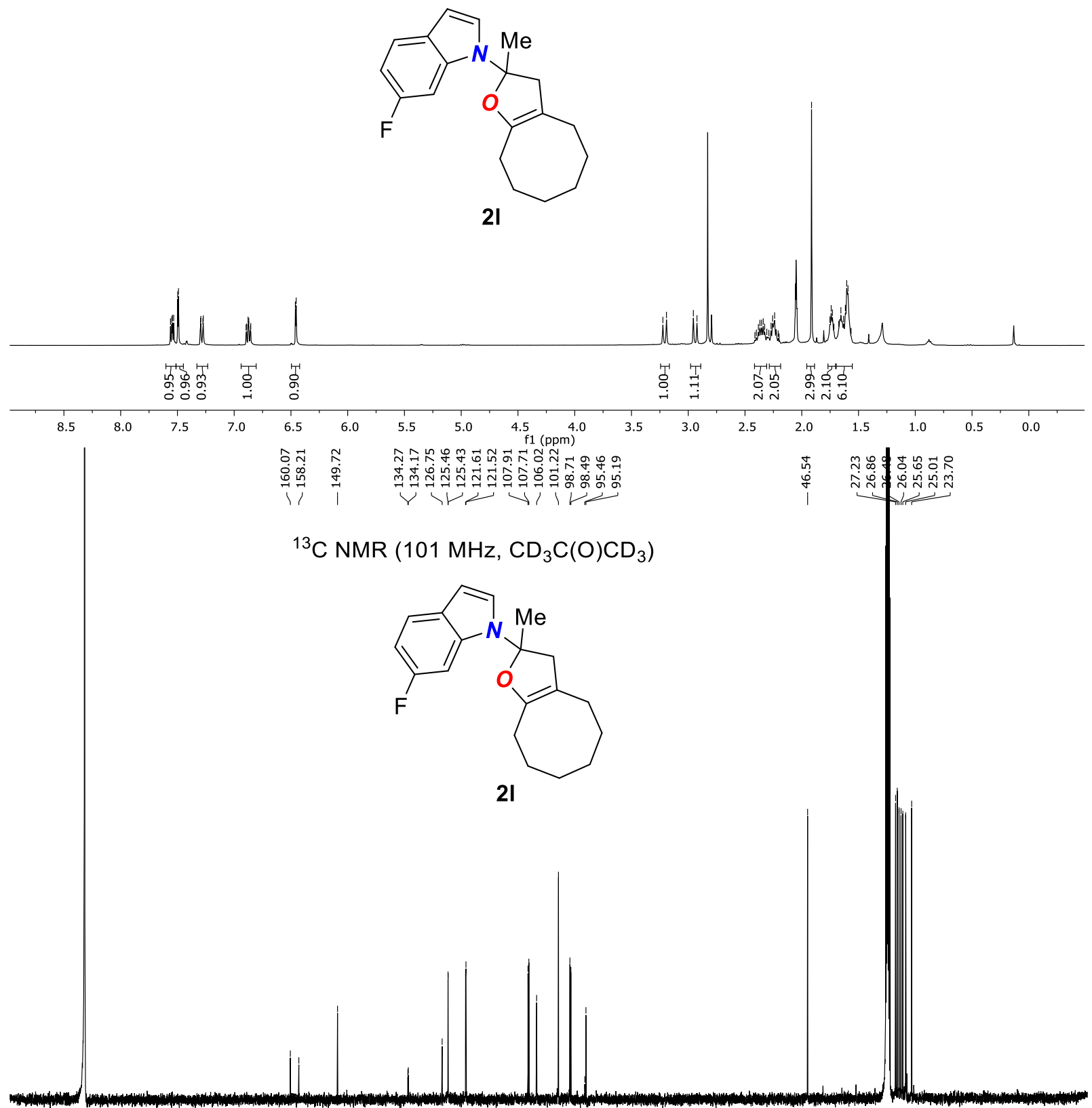

$\begin{array}{llllllllllll}220 & 210 & 200 & 190 & 180 & 170 & 160 & 150 & 140 & 130 & 120 & 110 \begin{array}{l}100 \\ \mathrm{f} 1(\mathrm{ppm})\end{array}\end{array}$

SI-71 
${ }^{19} \mathrm{~F}$ NMR $\left(376 \mathrm{MHz}, \mathrm{CD}_{3} \mathrm{C}(\mathrm{O}) \mathrm{CD}_{3}\right)$

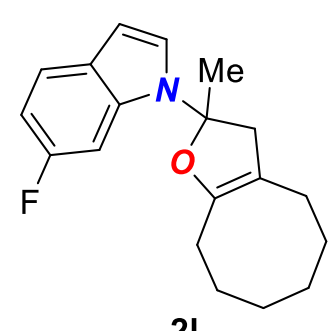

2I

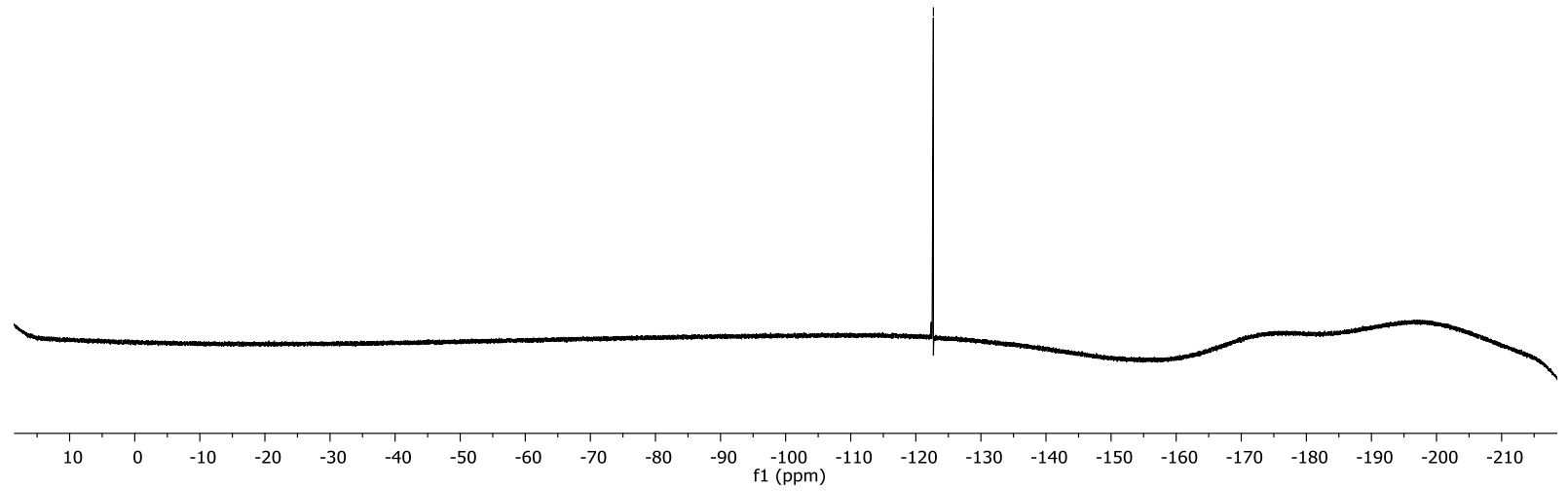




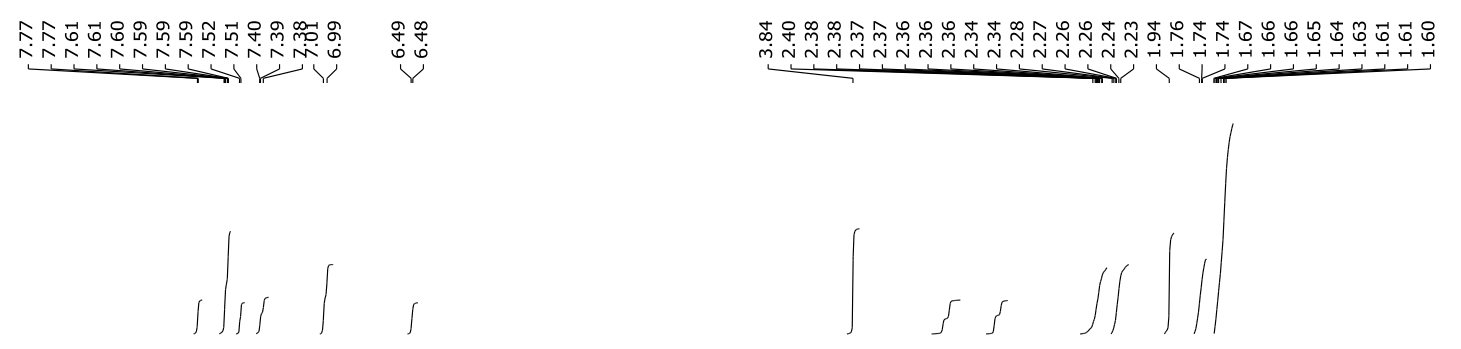

${ }^{1} \mathrm{H}$ NMR $\left(400 \mathrm{MHz}, \mathrm{CD}_{3} \mathrm{C}(\mathrm{O}) \mathrm{CD}_{3}\right)$

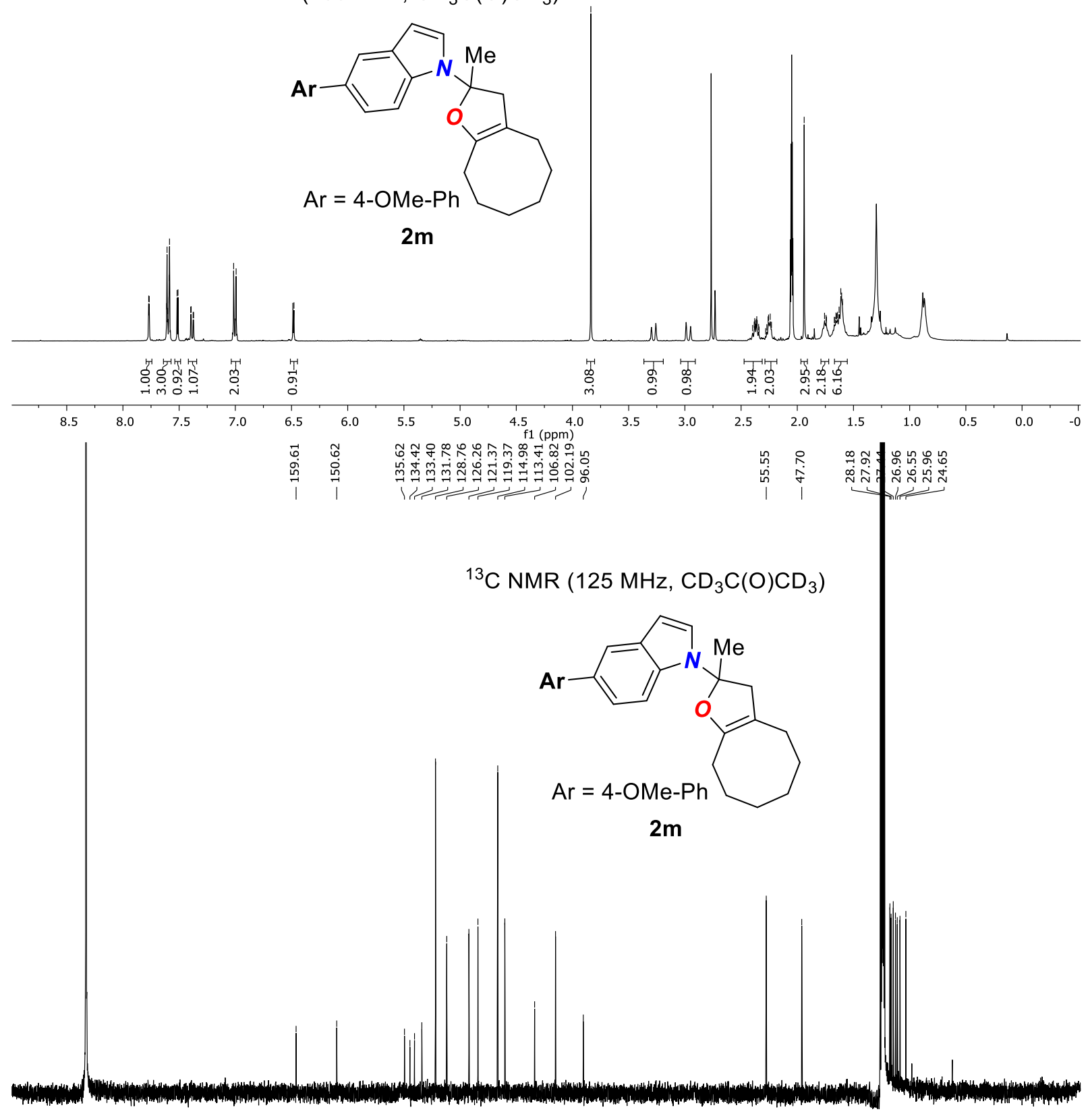

$\begin{array}{llllllllllllllllllllllllllllllllll}220 & 210 & 200 & 190 & 180 & 170 & 160 & 150 & 140 & 130 & 120 & 110 & 100 & 90 & 80 & 70 & 60 & 50 & 40 & 30 & 20 & 10 & 0 & -10\end{array}$ SI-73 
$1 / 11+1 / 1)$

${ }^{1} \mathrm{H}$ NMR $\left(400 \mathrm{MHz}, \mathrm{CD}_{3} \mathrm{C}(\mathrm{O}) \mathrm{CD}_{3}\right)$
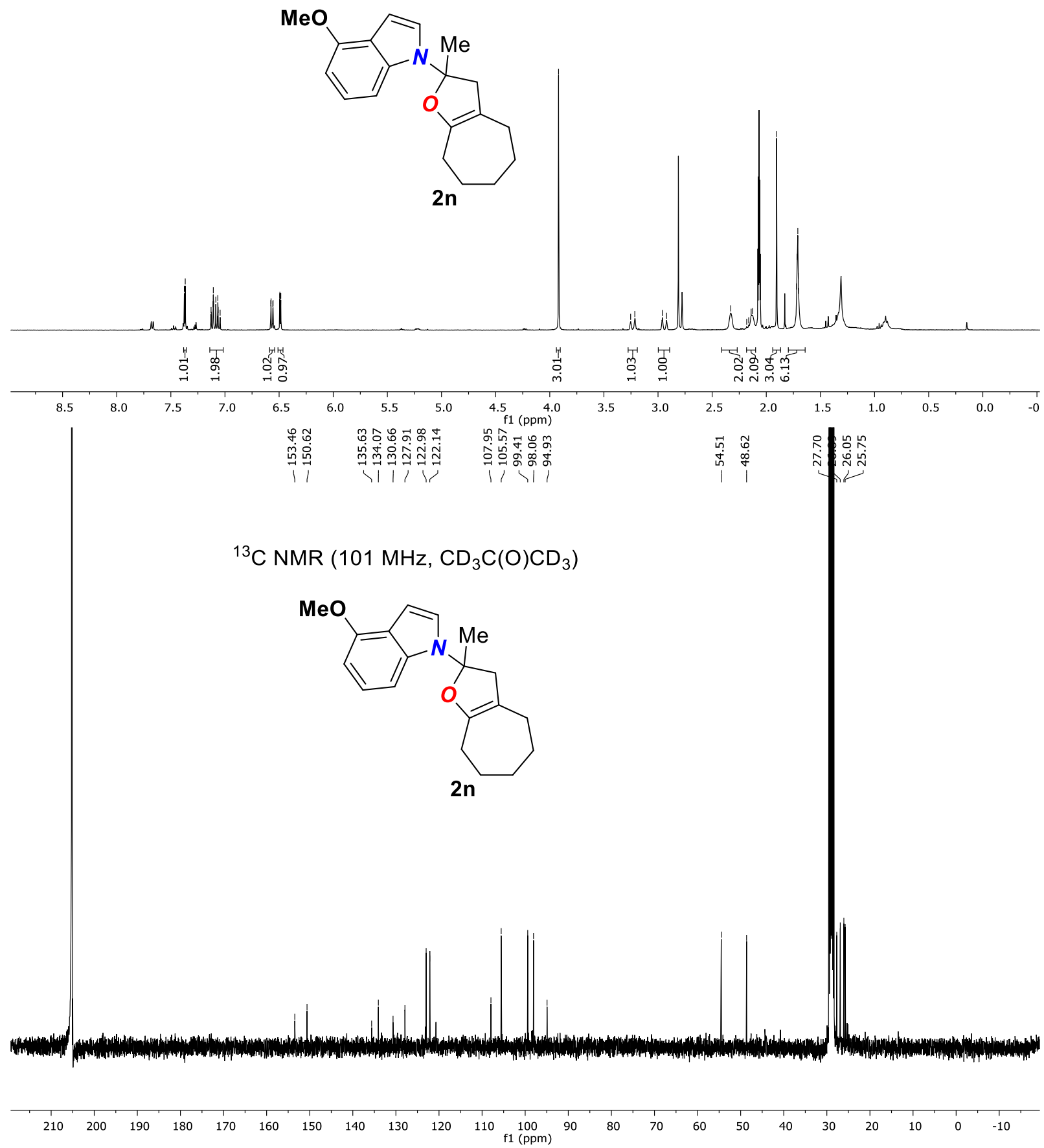

SI-74 


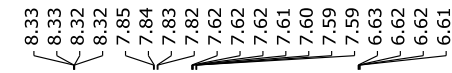

111
|

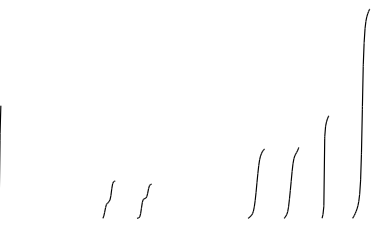

${ }^{1} \mathrm{H}$ NMR $\left(400 \mathrm{MHz}, \mathrm{CD}_{3} \mathrm{C}(\mathrm{O}) \mathrm{CD}_{3}\right)$

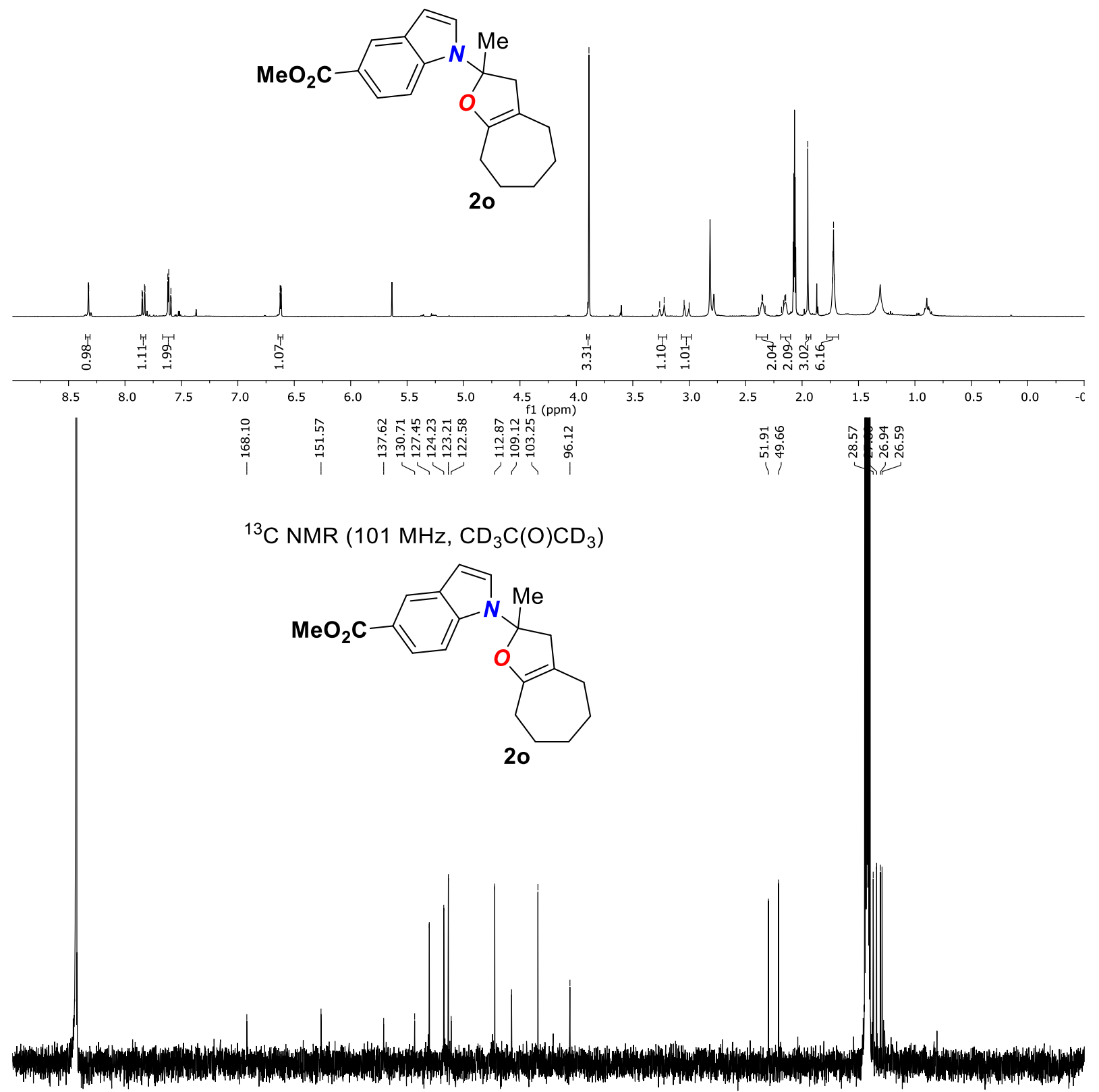

20

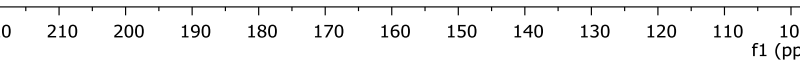

SI-75 


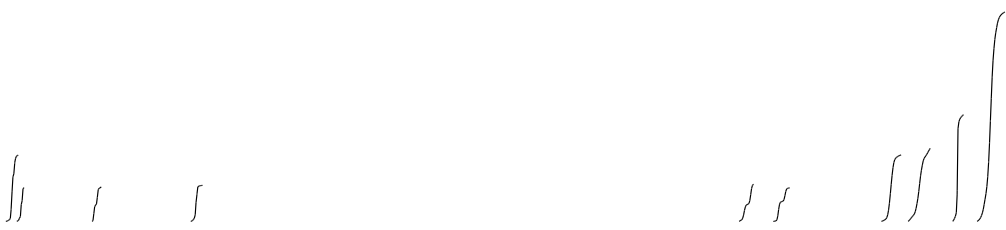

${ }^{1} \mathrm{H}$ NMR $\left(400 \mathrm{MHz}, \mathrm{CD}_{3} \mathrm{C}(\mathrm{O}) \mathrm{CD}_{3}\right)$

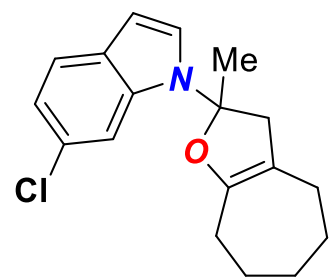

$2 p$
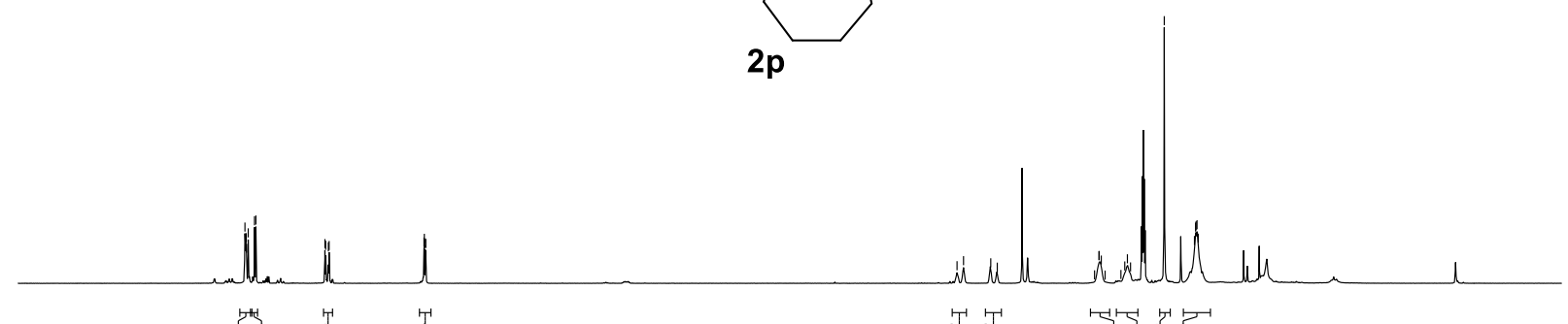

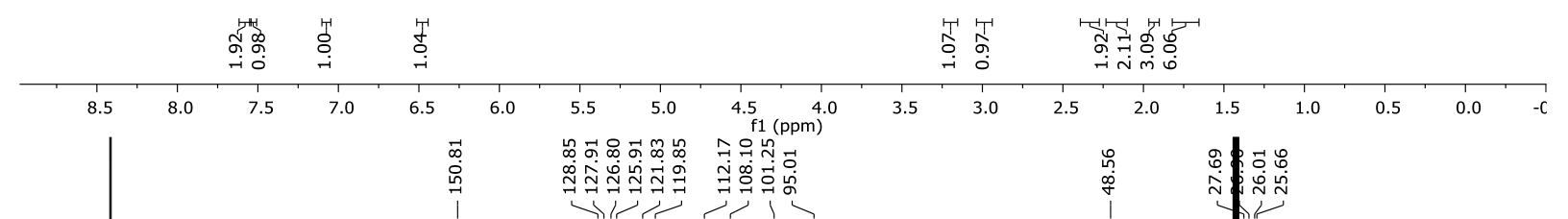

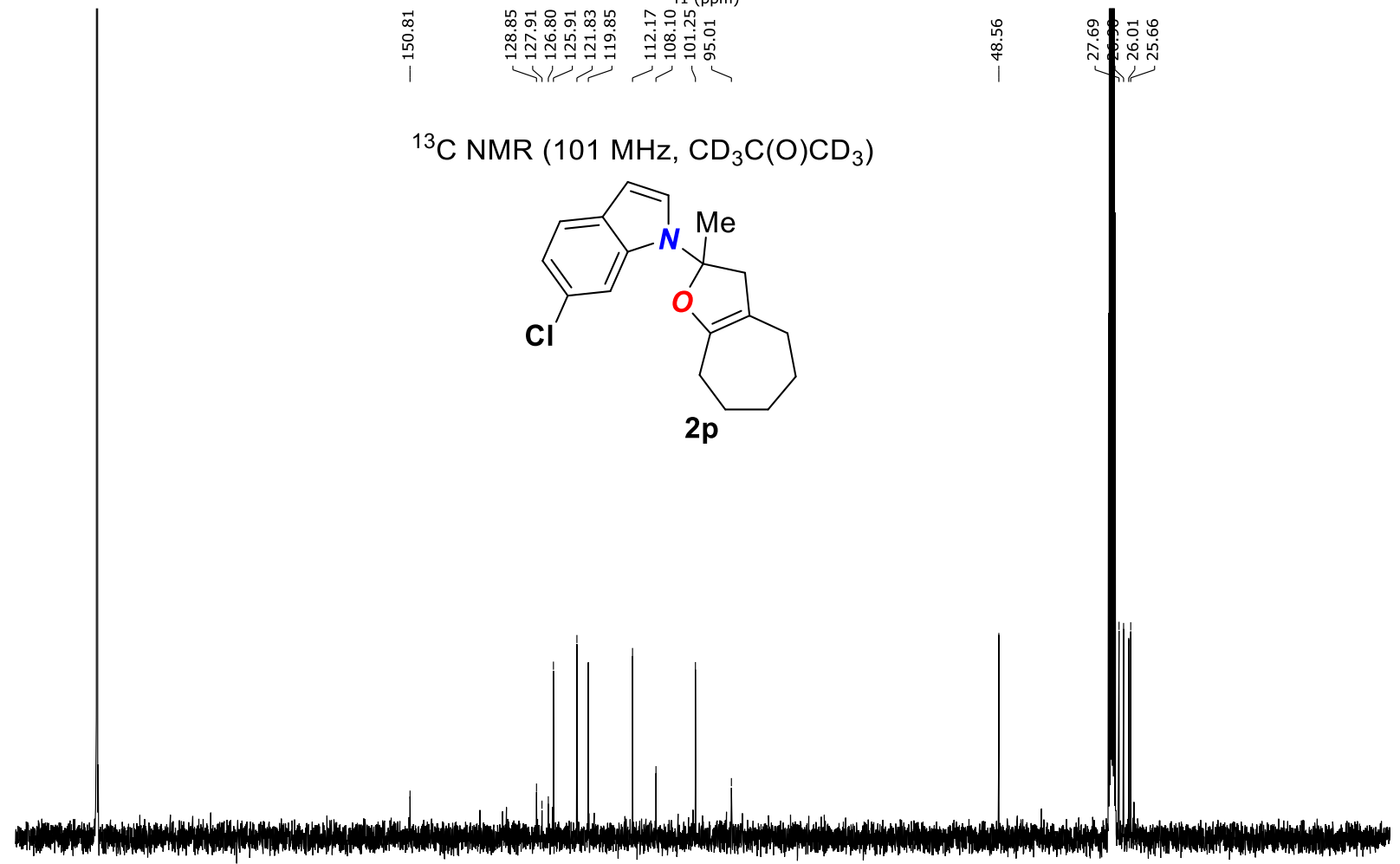

$\begin{array}{lllllllllllllllllllllllll}210 & 200 & 190 & 180 & 170 & 160 & 150 & 140 & 130 & 120 & 110 & 100 & 90 & 80 & 70 & 60 & 50 & 40 & 30 & 20 & 10 & 0 & -10\end{array}$

SI-76 